ANALYSIS \& PDE

Volume $3 \quad$ No. $3 \quad 2010$

\author{
JIAN SONG AND STEVE ZELDITCH
}

BERGMAN METRICS AND GEODESICS IN THE SPACE OF KÄHLER METRICS ON TORIC VARIETIES 


\title{
BERGMAN METRICS AND GEODESICS IN THE SPACE OF KÄHLER METRICS ON TORIC VARIETIES
}

\author{
JIAN SONG AND STEVE ZELDITCH
}

\begin{abstract}
A guiding principle in Kähler geometry is that the infinite-dimensional symmetric space $\mathscr{H}$ of Kähler metrics in a fixed Kähler class on a polarized projective Kähler manifold $M$ should be well approximated by finite-dimensional submanifolds $\mathscr{B}_{k} \subset \mathscr{H}$ of Bergman metrics of height $k$ (Yau, Tian, Donaldson). The Bergman metric spaces are symmetric spaces of type $G_{\mathbb{C}} / G$ where $G=U\left(d_{k}+1\right)$ for certain $d_{k}$. This article establishes some basic estimates for Bergman approximations for geometric families of toric Kähler manifolds.

The approximation results are applied to the endpoint problem for geodesics of $\mathscr{H}$, which are solutions of a homogeneous complex Monge-Ampère equation in $A \times X$, where $A \subset \mathbb{C}$ is an annulus. Donaldson, Arezzo and Tian, and Phong and Sturm raised the question whether $\mathscr{H}$-geodesics with fixed endpoints can be approximated by geodesics of $\mathscr{P}_{k}$. Phong and Sturm proved weak $C^{0}$-convergence of Bergman to Monge-Ampère geodesics on a general Kähler manifold. Our approximation results show that one has $C^{2}(A \times X)$ convergence in the case of toric Kähler metrics, extending our earlier result on $\mathbb{C P}^{1}$.
\end{abstract}

1. Introduction

2. Background on toric varieties $\quad 308$

3. The functions $\mathscr{P}_{h^{k}}$ and $\mathscr{2}_{h^{k}} \quad 317$

4. The Szegó kernel of a toric variety 320

5. Localization of sums: proof of the Localization Lemma $1.2 \quad 326$

6. Proof of the Regularity Lemma 1.3 and joint asymptotics of $\mathscr{P}_{h^{k}}(\alpha) \quad 330$

7. $C^{0}$ and $C^{1}$-convergence $\quad 348$

8. $C^{2}$-convergence $\quad 351$

Acknowledgments $\quad 356$

$\begin{array}{ll}\text { References } & 356\end{array}$

\section{Introduction}

This is the first in a series of articles on the Riemannian geometry of the space

$$
\mathscr{H}=\left\{\varphi \in C^{\infty}(M): \omega_{\varphi}=\omega_{0}+d d^{c} \varphi>0\right\}
$$

of Kähler metrics in the class $\left[\omega_{0}\right]$ of a polarized projective Kähler manifold $\left(M, \omega_{0}, L\right)$, equipped with

MSC2000: 14M25, 35P20, 35S30, 53C22, 53C55.

Keywords: Kähler metrics, Bergman kernels, toric varieties, Monge-Ampère.

Research partially supported by National Science Foundation grants DMS-0604805 and DMS-0904252. 
the Riemannian metric $g_{\mathscr{H}}$ of Mabuchi [1987], Semmes [1992], and Donaldson [1999]:

$$
\|\psi\|_{g_{\mathscr{H}}, \varphi}^{2}=\int_{M}|\psi|^{2} \frac{\omega_{\varphi}^{m}}{m !}, \quad \text { where } \varphi \in \mathscr{H} \text { and } \psi \in T_{\varphi} \mathscr{H} \simeq C^{\infty}(M) .
$$

Here, $L \rightarrow M$ is an ample line bundle with $c_{1}(L)=\left[\omega_{0}\right]$. Formally, $\left(\mathcal{H}, g_{\mathscr{H}}\right)$ is an infinite-dimensional nonpositively curved symmetric space of the type $G_{\mathbb{C}} / G$, where $G=\operatorname{SDiff}_{\omega_{0}}(M)$ is the group of Hamiltonian symplectic diffeomorphisms of $\left(M, \omega_{0}\right)$. This statement is only formal since $G$ does not possess a complexification and $\mathscr{H}$ is an incomplete, infinite-dimensional space. An attractive approach to the infinite-dimensional geometry is to approximate it by a sequence of finite-dimensional submanifolds $\mathscr{B}_{k} \subset \mathscr{H}$ of so-called Bergman (or Fubini-Study) metrics. The space $\mathscr{S}_{k}$ of Bergman metrics may be identified with the finite-dimensional symmetric space $\operatorname{GL}\left(d_{k}+1, \mathbb{C}\right) / U\left(d_{k}+1\right)$, where $d_{k}$ is a certain dimension. Thus, $\mathscr{B}_{k}$ is equipped with a finite-dimensional symmetric space metric $g_{\mathscr{B}_{k}}$, which is not the same as the submanifold Riemannian metric induced on it by $g_{\mathscr{H}}$. The purpose of the series is to show that much of the symmetric space geometry of $\left(\mathscr{P}_{k}, g_{\mathscr{B}_{k}}\right)$ tends to the infinite-dimensional symmetric space geometry of $\left(\mathcal{H}, g_{\mathscr{H}}\right)$ as $k \rightarrow \infty$.

To put the problem and results in perspective, we recall that at the level of individual metrics $\omega \in \mathscr{H}$, there exists a well-developed approximation theory: Given $\omega$, one can define a canonical sequence of Bergman metrics $\omega_{k} \in \mathscr{B}_{k}$ which approximates $\omega$ in the $C^{\infty}$ topology (see (9)), in much the same way that smooth functions can be approximated by Bernstein polynomials [Yau 1992; Tian 1990]; see also [Catlin 1999; Zelditch 1998; 2009]. The approximation theory is based on microlocal analysis in the complex domain, specifically Bergman kernel asymptotics on and off the diagonal [Boutet de Monvel and Sjöstrand 1976; Catlin 1999; Zelditch 1998; Donaldson 2001; Phong and Sturm 2009]. The same methods are used in [Rubinstein and Zelditch $\geq 2010 \mathrm{a}$ ] to prove that the geometry of $\left(\mathscr{P}_{k}, g_{\mathscr{P}_{k}}\right)$ tends to the geometry of $\left(\mathscr{H}, g_{\mathscr{H}}\right)$ at the infinitesimal level: for example, that the Riemann metric, connection and curvature tensor of $\mathscr{B}_{k}$ tend to the Riemann metric, connection and curvature of $\mathscr{H}$. But our principal aim in this series is to extend the approximation from pointwise or infinitesimal objects to more global aspects of the geometry, such as $\mathscr{B}_{k}$-geodesics or harmonic maps to $\left(\mathscr{S}_{k}, g_{\mathscr{B}_{k}}\right)$. These more global approximation problems are much more difficult than the infinitesimal ones. The obstacles are analogous to those involved in complexifying $\operatorname{SDiff}_{\omega_{0}}(M)$. We will explain this comparison in more detail in Section $1 \mathrm{~F}$ at the end of this introduction.

This article is concerned with the approximation of $g_{\mathscr{H}}$-geodesic segments $\omega_{t}$ in $\mathscr{H}$ with fixed endpoints by $g_{\mathscr{B}_{k}}$-geodesic segments in $\mathscr{B}_{k}$. As recalled in Section $1 \mathrm{~A}$, the geodesic equation for the Kähler potentials $\varphi_{t}$ of $\omega_{t}$ is a complex homogeneous Monge-Ampère equation. Little is known about the solutions of the Dirichlet problem at present beyond the regularity result that $\varphi_{t} \in C^{1, \alpha}([0, T] \times M)$ for all $\alpha<1$ if the endpoint metrics are smooth (see [Chen 2000; Chen and Tian 2008] for results and background). It is therefore natural to study the approximation of Monge-Ampère $g_{\mathscr{H}}$-geodesics $\varphi_{t}$ by the much simpler $g_{\mathscr{B}_{k}}$-geodesics $\varphi_{k}(t, z)$, which are defined by one-parameter subgroups of $\operatorname{GL}\left(d_{k}+1, \mathbb{C}\right)$ (see (24)). The problem of approximating $\mathscr{H}$-geodesic segments between two smooth endpoints by $\mathscr{B}_{k}$ geodesic segments was raised by Donaldson [2001], Arezzo and Tian [2003] and Phong and Sturm [2006] and was studied in depth by Phong and Sturm [2006; 2007]. Phong and Sturm [2006] proved that $\varphi_{k}(t, z) \rightarrow \varphi_{t}$ in a weak $C^{0}$ sense on $[0,1] \times M$ (see (13)); a $C^{0}$ result with a remainder estimate was later proved by Berndtsson [2009] for a somewhat different approximation. 
In this article, we study the $g_{\mathscr{B}_{k}}$-approximation of $g_{\mathscr{H}}$-geodesics in the case of a polarized projective toric Kähler manifold. Our main result is that a $g_{\mathscr{H}}$ geodesic segment of toric Kähler metrics with fixed endpoints is approximated in $C^{2}$ by a sequence $\varphi_{k}(t, z)$ of toric $g_{\mathscr{S}_{k}}$-geodesic segments. More precisely, for any $T \in \mathbb{R}_{+}$, we have $\varphi_{k}(t, z) \rightarrow \varphi_{t}(z)$ in $C^{2}([0, T] \times M)$, generalizing the results of Song and Zelditch [2007a] in the case of $\mathbb{C P}^{1}$. It is natural to study convergence of two (space-time) derivatives since the Kähler metric $\omega_{\varphi}=\omega_{0}+d d^{c} \varphi$ involves two derivatives. In the course of the proof, we introduce methods which have many other applications to global approximation problems on toric Kähler manifolds, and which should also have applications to nontoric Kähler manifolds.

Here, as in [Song and Zelditch 2007b; Rubinstein and Zelditch 2010; $\geq 2010 \mathrm{~b}$ ], we restrict to the toric setting because, at this stage, it is possible to obtain much stronger results than for general Kähler manifolds and because it is one of the few settings where we can see clearly what is involved in the classical limit as $k \rightarrow \infty$. The simplifying feature of toric Kähler manifolds is that they are completely integrable on both the classical and quantum level. In Riemannian terms, the submanifolds of toric metrics of $\mathscr{H}$ and $\mathscr{B}_{k}$ form totally geodesic flats. Hence in the toric case, the geodesic equation along the flat is linearized by the Legendre transform, with the consequence that there exists an explicit formula for the Monge-Ampère geodesic $\varphi_{t}$ between two smooth toric endpoint metrics. In particular, the explicit formula shows that geodesics between smooth toric endpoints are smooth. We use this explicit solution throughout the article, starting from (29). Thus, in the toric case we only need to prove $C^{2}$-convergence of the Bergman approximation. An analogous result on a general Kähler manifold would require an improvement on the known regularity results on Monge-Ampère geodesics in addition to a convergence result. We refer to [Chen and Tian 2008] for the state of the art on the regularity theory.

1A. Background. To state our results, we need some notation and background. Let $L \rightarrow M^{m}$ be an ample holomorphic line bundle over a compact complex manifold of dimension $m$. Let $\omega_{0} \in H^{(1,1)}(M, \mathbb{Z})$ denote an integral Kähler form. Fixing a reference hermitian metric $h_{0}$ on $L$, we may write other hermitian metrics on $L$ as

$$
h_{\varphi}=e^{-\varphi} h_{0},
$$

and then the space of hermitian metrics $h$ on $L$ with curvature $(1,1)$-forms $\omega_{h}$ in the class of $\omega_{0}$ may (by the $\partial \bar{\partial}$ lemma) be identified with the space $\mathscr{H}$ of relative Kähler potentials (1). We may then identify the tangent space $T_{\varphi} \mathscr{H}$ at $\varphi \in \mathscr{H}$ with $C^{\infty}(M)$. Following [Mabuchi 1987; Semmes 1992; Donaldson 2001], we define the Riemannian metric (2) on $\mathscr{H}$. With this Riemannian metric, $\mathscr{H}$ is formally an infinite-dimensional nonpositively curved symmetric space.

The space $\mathscr{P}_{k}$ of Bergman (or Fubini-Study) metrics of height $k$ is defined as follows: Let $H^{0}\left(M, L^{k}\right)$ denote the space of holomorphic sections of the $k$-th power $L^{k} \rightarrow M$ of $L$ and let $d_{k}+1=\operatorname{dim} H^{0}\left(M, L^{k}\right)$. We let $\mathscr{B} H^{0}\left(M, L^{k}\right)$ denote the manifold of all bases $\underline{s}=\left\{s_{0}, \ldots, s_{d_{k}}\right\}$ of $H^{0}\left(M, L^{k}\right)$. Given a basis, we define the Kodaira embedding

$$
l_{\underline{s}}: M \rightarrow \mathbb{C P}^{d_{k}}, \quad z \mapsto\left[s_{0}(z), \ldots, s_{d_{k}}(z)\right] .
$$

We then define a Bergman metric (or equivalently, Fubini-Study) metric of height $k$ to be a metric of the form

$$
h_{\underline{s}}:=\left(l_{\underline{s}}^{*} h_{\mathrm{FS}}\right)^{1 / k}=\frac{h_{0}}{\left(\sum_{j=0}^{d_{k}}\left|s_{j}(z)\right|_{h_{0}^{k}}^{2}\right)^{1 / k}},
$$


where $h_{\mathrm{FS}}$ is the Fubini-Study hermitian metric on $\mathbb{O}(1) \rightarrow \mathbb{C P}^{d_{k}}$. We then define

$$
\mathscr{B}_{k}=\left\{h_{\underline{s}}: \underline{s} \in \mathscr{B} H^{0}\left(M, L^{k}\right)\right\} .
$$

Here, $h_{0}^{k}$ is the $k$-th tensor power of the Hermitian metric $h_{0}$ on $\mathcal{O}(1)$ and $h_{\underline{s}}$ is independent of the choice of $h_{0}$. We use the same notation for the associated space of potentials $\varphi$ such that $h_{\underline{s}}=e^{-\varphi} h_{0}$ and for the associated Kähler metrics $\omega_{\varphi}$. We observe that with a choice of basis of $H^{0}\left(M, L^{\bar{k}}\right)$ we may identify $\mathscr{B}_{k}$ with the symmetric space $\mathrm{GL}\left(d_{k}+1, \mathbb{C}\right) / U\left(d_{k}+1\right)$ since $\mathrm{GL}\left(d_{k}+1, \mathbb{C}\right)$ acts transitively on the set of bases, while $l_{\underline{s}}^{*} h_{\mathrm{FS}}$ is unchanged if we replace the basis $\underline{s}$ by a unitary change of basis.

Several further identifications are important. The first is that $\mathscr{B}_{k}$ may be identified with the space $\Phi_{k}$ of hermitian inner products on $H^{0}\left(M, L^{k}\right)$, the correspondence being that a basis is identified with an inner product for which the basis is hermitian orthonormal. As in [Donaldson 2001; 2005], we define maps

$$
\operatorname{Hilb}_{k}: \mathscr{H} \rightarrow \Phi_{k},
$$

by the rule that a hermitian metric $h \in \mathscr{H}$ induces the inner products on $H^{0}\left(M, L^{k}\right)$,

$$
\|s\|_{\text {Hilb }_{k}(h)}^{2}=R \int_{M}|s(z)|_{h^{k}}^{2} d V_{h}, \quad \text { where } d V_{h}=\frac{\omega_{h}^{m}}{m !} \text { and } R=\frac{d_{k}+1}{\operatorname{Vol}\left(M, d V_{h}\right)} .
$$

Further, we define the identifications

$$
\mathrm{FS}_{k}: \mathscr{I}_{k} \simeq \mathscr{P}_{k}
$$

as follows: an inner product $G=\langle$,$\rangle on H^{0}\left(M, L^{k}\right)$ determines a $G$-orthonormal basis $\underline{s}=\underline{s}_{G}$ of $H^{0}\left(M, L^{k}\right)$ and an associated Kodaira embedding (3) and Bergman metric (4). Thus,

$$
\mathrm{FS}_{k}(G)=h_{\underline{s}_{G}} .
$$

The right side is independent of the choice of $h_{0}$ and the choice of orthonormal basis. As observed in [Donaldson 2001; Phong and Sturm 2006], $\mathrm{FS}_{k}(G)$ is characterized by the fact that for any $G$ orthonormal basis $\left\{s_{j}\right\}$ of $H^{0}\left(M, L^{k}\right)$, we have

$$
\sum_{j=0}^{d_{k}}\left|s_{j}(z)\right|_{\mathrm{FS}_{k}(G)}^{2} \equiv 1 \quad \text { for all } z \in M .
$$

Metrics in $\mathscr{B}_{k}$ are defined by an algebro-geometric construction. By analogy with the approximation of real numbers by rational numbers, we say that $h \in \mathscr{H}$ (or its curvature form $\omega_{h}$ ) has height $k$ if $h \in \mathscr{P}_{k}$. A basic fact is that the union

$$
\mathscr{B}=\bigcup_{k=1}^{\infty} \mathscr{B}_{k}
$$

of Bergman metrics is dense in the $C^{\infty}$-topology in the space $\mathscr{H}$ [Tian 1990; Zelditch 1998]. Indeed,

$$
\frac{\mathrm{FS}_{k} \circ \operatorname{Hilb}_{k}(h)}{h}=1+O\left(k^{-2}\right),
$$

where the remainder is estimated in $C^{r}(M)$ for any $r>0$; the left side moreover has a complete asymptotic expansion. See [Donaldson 2002; Phong and Sturm 2006] for precise statements. 
Now that we have defined the spaces $\mathscr{H}$ and $\mathscr{B}_{k}$, we can compare Monge-Ampère geodesics and Bergman geodesics. Geodesics of $\mathscr{H}$ satisfy the Euler-Lagrange equations for the energy functional determined by (2); see (68). By [Mabuchi 1987; Semmes 1992; Donaldson 1999], the geodesics of $\mathscr{H}$ in this metric are the paths $h_{t}=e^{-\varphi_{t}} h_{0}$ which satisfy the equation

$$
\ddot{\varphi}-\frac{1}{2}|\nabla \dot{\varphi}|_{\omega_{\varphi}}^{2}=0
$$

which may be interpreted as a homogeneous complex Monge-Ampère equation on $A \times M$, where $A$ is an annulus [Semmes 1992; Donaldson 1999].

Geodesics in $\mathscr{B}_{k}$ with respect to the symmetric space metric are given by orbits of certain oneparameter subgroups $\sigma_{k}^{t}=e^{t A_{k}}$ of $\mathrm{GL}\left(d_{k}+1, \mathbb{C}\right)$. In the identification of $\mathscr{S}_{k}$ with the symmetric space $\Phi_{k} \simeq \mathrm{GL}\left(d_{k}+1, \mathbb{C}\right) / U\left(d_{k}+1\right)$ of inner products, the one-parameter subgroup $e^{t A_{k}} \in \mathrm{GL}\left(d_{k}+1\right)$ changes an orthonormal basis $\underline{\hat{s}}^{(0)}$ for the initial inner product $G_{0}$ to an orthonormal basis $e^{t A_{k}} \cdot \underline{\hat{s}}^{(0)}$ for $G_{t}$, where $G_{t}$ is a geodesic of $\mathscr{I}_{k}$. Geometrically, a Bergman geodesic may be visualized as the path of metrics on $M$ obtained by holomorphically embedding $M$ using a basis of $H^{0}\left(M, L^{k}\right)$ and then moving the embedding under the one-parameter subgroup $e^{t A_{k}}$ of motions of $\mathbb{C P}^{d_{k}}$. The difficulty is to interpret this simple extrinsic motion in intrinsic terms on $M$.

In this article, we only study the endpoint problem for the geodesic equation. We are given $h_{0}, h_{1} \in$ $\mathscr{H}$ and let $h(t)$ denote the Monge-Ampère geodesic between them. We then consider the geodesic $G_{k}(t)$ of $\mathscr{I}_{k}$ between $G_{k}(0)=\operatorname{Hilb}_{k}\left(h_{0}\right)$ and $G_{k}(1)=\operatorname{Hilb}_{k}\left(h_{1}\right)$ or equivalently between $\mathrm{FS}_{k} \circ \mathrm{Hilb}_{k}\left(h_{0}\right)$ and $\mathrm{FS}_{k} \circ \mathrm{Hilb}_{k}\left(h_{1}\right)$. Without loss of generality, we may assume that the change of orthonormal basis (or change of inner product) matrix $\sigma_{k}=e^{A_{k}}$ between $\operatorname{Hilb}_{k}\left(h_{0}\right), \operatorname{Hilb}_{k}\left(h_{1}\right)$ is diagonal with entries $e^{\lambda_{0}}, \ldots, e^{\lambda_{d_{k}}}$ for some $\lambda_{j} \in \mathbb{R}$. Let $\underline{\hat{s}}^{(t)}=e^{t A_{k}} \cdot \underline{\hat{s}}^{(0)}$, where $e^{t A_{k}}$ is diagonal with entries $e^{\lambda_{j} t}$. Define

$$
h_{k}(t):=\mathrm{FS}_{k} \circ G_{k}(t)=h_{\hat{\underline{s}}^{(t)}}=: h_{0} e^{-\varphi_{k}(t)} .
$$

It follows immediately from (8) that

$$
\varphi_{k}(t ; z)=\frac{1}{k} \log \sum_{j=0}^{d_{k}} e^{2 \lambda_{j} t}\left|\hat{s}_{j}^{(0)}\right|_{h_{0}^{k}}^{2} .
$$

We emphasize that $\varphi_{k}(t ; z)$ is the intrinsic $\mathscr{B}_{k}$ geodesic between the endpoints $\mathrm{FS}_{k} \circ \mathrm{Hilb}_{k}\left(h_{0}\right)$ and $\mathrm{FS}_{k} \circ \mathrm{Hilb}_{k}\left(h_{1}\right)$. It is of course quite distinct from the Hilb ${ }_{k}$-image of the Monge-Ampère geodesic; the latter is not intrinsic to $\mathscr{B}_{k}$ and one cannot gain any information on the $\mathscr{H}$-geodesic by studying it.

We summarize the notation for hermitian metrics and geodesics of metrics:

- For any metric $h$ on $L, h^{k}$ denotes the induced metric on $L^{k}$, and for any metric $H$ on $L^{k}, H^{1 / k}$ is the induced metric on $L$;

- Given $h_{0} \in \mathscr{H}, h_{t}=e^{-\varphi_{t}} h_{0}$ is the Monge-Ampère geodesic;

- $h_{k}=\mathrm{FS} \circ \operatorname{Hilb}_{k}(h) \in \mathscr{M}_{k}$ is the natural approximating Bergman metric to $h$, and $h_{k}(t)=e^{-\varphi_{k}(t)} h_{0}$ is the Bergman geodesic (11).

The main result of [Phong and Sturm 2006] is that the Monge-Ampère geodesic $\varphi_{t}$ is approximated by the one-parameter subgroup Bergman geodesic $\varphi_{k}(t, z)$ in the following weak $C^{0}$ sense: 


$$
\varphi_{t}(z)=\lim _{\ell \rightarrow \infty}\left[\sup _{k \geq \ell} \varphi_{k}(t, z)\right]^{*} \quad \text { uniformly as } \ell \rightarrow \infty,
$$

where $u^{*}$ is the upper envelope of $u$, that is, $u^{*}\left(\zeta_{0}\right)=\lim _{\epsilon \rightarrow 0} \sup _{\left|\zeta-\zeta_{0}\right|<\epsilon} u(\zeta)$. In particular, without taking the upper envelope, $\sup _{k \geq \ell} \varphi_{k}(t, z) \rightarrow \varphi(t, z)$ almost everywhere as $\ell \rightarrow \infty$. See also [Berndtsson 2009] for the subsequent proof of an analogous result for the adjoint bundle $L^{k} \otimes K$ (where $K$ is the canonical bundle) with an error estimate $\left\|\varphi_{k}(t)-\varphi(t)\right\|_{C^{0}}=O(\log k / k)$.

1B. Statement of results. Our purpose is to show that the degree of convergence of $h_{k}(t) \rightarrow h_{t}$ or equivalently of $\varphi_{k}(t, z) \rightarrow \varphi_{t}(z)$ is much stronger that $C^{0}$ for toric hermitian metrics on the invariant line bundle $L \rightarrow M$ over a smooth toric Kähler manifold. We recall that a toric variety $M$ of dimension $m$ carries the holomorphic action of a complex torus $\left(\mathbb{C}^{*}\right)^{m}$ with an open dense orbit. The associated real torus $\mathbf{T}^{m}=\left(S^{1}\right)^{m}$ acts on $M$ in a Hamiltonian fashion with respect to any invariant Kähler metric $\omega$, that is, it possesses a moment map

$$
\mu: M \rightarrow P
$$

with image a convex lattice polytope. Here, and henceforth, $P$ denotes the closed polytope; its interior is denoted $P^{o}$ (see Section 2 for background). Objects associated to $M$ are called toric if they are invariant or equivariant with respect to the torus action (real or complex, depending on the context). We define the space of toric hermitian metrics by

$$
\mathscr{H}_{\mathbf{T}^{m}}=\left\{\varphi \in \mathscr{H}:\left(e^{i \theta}\right)^{*} \varphi=\varphi \text { for all } e^{i \theta} \in \mathbf{T}^{m}\right\} .
$$

Here, we assume the reference metric $h_{0}$ is $\mathbf{T}^{m}$-invariant. We note that since $\mathbf{T}^{m}$ has a moment map, it automatically lifts to $L$ and hence it makes sense to say that $h_{0}: L \rightarrow \mathbb{C}$ is invariant under it. With a slight abuse of notation carried over from [Donaldson 2001], we also let $\varphi$ denote the full Kähler potential on the open orbit, that is, $\omega_{\varphi}=d d^{c} \varphi$ on the open orbit. It is clearly $\mathbf{T}^{m}$-invariant.

Our main result is:

Theorem 1.1. Let $L \rightarrow M$ be a very ample toric line bundle over a smooth compact toric variety $M$. Let $\mathscr{H}_{T}$ denote the space of toric hermitian metrics on L. Let $h_{0}, h_{1} \in \mathscr{H}_{T}$ and let $h_{t}$ be the Monge-Ampère geodesic between them. Let $h_{k}(t)$ be the Bergman geodesic between $\mathrm{Hilb}_{k}\left(h_{0}\right)$ and $\mathrm{Hilb}_{k}\left(h_{1}\right)$ in $\mathscr{B}_{k}$. Let $h_{k}(t)=e^{-\varphi_{k}(t, z)} h_{0}$ and let $h_{t}=e^{-\varphi_{t}(z)} h_{0}$. Then

$$
\lim _{k \rightarrow \infty} \varphi_{k}(t, z)=\varphi_{t}(z)
$$

in $C^{2}([0,1] \times M)$. In fact, there exists $C$ independent of $k$ such that

$$
\left\|\varphi_{k}-\varphi\right\|_{C^{2}([0,1] \times M)} \leq C k^{-1 / 3+\epsilon} \quad \text { for all } \epsilon>0 .
$$

Our methods show moreover that away from the divisor at infinity $\mathscr{D}$ (see Section 2), the function $\varphi_{k}(t, z)$ has an asymptotic expansion in powers of $k^{-1}$, and converges in $C^{\infty}$ to $\varphi_{t}$. But the asymptotics become complicated near $\mathscr{D}$, and require a "multiscale" analysis involving distance to boundary facets. It is therefore not clear whether $\varphi_{k}$ has an asymptotic expansion in $k^{-1}$ globally on $M$. At least, no such asymptotics follow from the known Bergman kernel asymptotics, on or off the diagonal. The analysis of these regimes for general toric varieties seems to be fundamental in "quantum mechanical approximations" on toric varieties. 
As mentioned above, the Monge-Ampère equation can be linearized in the toric case and solved explicitly (17); we give a simple new proof in Section 2. The geodesic arcs are easily seen to be $C^{\infty}$ when the endpoints are $C^{\infty}$. Hence the $C^{2}$-convergence result does not improve the known regularity results on Monge-Ampère geodesics of toric metrics, but pertains only to the degree of convergence of Bergman to Monge-Ampère geodesics in a setting where the latter are known to be smooth; it is possible that the methods can be developed to give regularity results, but this is a distant prospect (see the remarks at the end of this introduction).

1C. Outline of the proof. We now outline the proof of Theorem 1.1. We start with the fact that the Legendre transform of the Kähler potential linearizes the Monge-Ampère equation (see Section 2G and [Abreu 2003; Guan 1999; Donaldson 2002]). The Legendre transform $\mathscr{L} \varphi$ of the open-orbit Kähler potential $\varphi$, a convex function on $\mathbb{R}^{m}$ in logarithmic coordinates, is the so-called dual symplectic potential

$$
u_{\varphi}(x)=\mathscr{L} \varphi(x),
$$

a convex function on the convex polytope $P$. Under this Legendre transform, the complex MongeAmpère equation on $\mathscr{H}_{\mathbf{T}^{m}}$ linearizes to the equation $\ddot{u}=0$ and is thus solved by

$$
u_{t}=u_{\varphi_{0}}+t\left(u_{\varphi_{1}}-u_{\varphi_{0}}\right) .
$$

Hence the solution $\varphi_{t}$ of the geodesic equation on $\mathscr{H}$ is solved in the toric setting by

$$
\varphi_{t}=\mathscr{L}^{-1} u_{t} .
$$

Our goal is to show that $\varphi_{k}(t ; z) \rightarrow \mathscr{L}^{-1} u_{t}$ as in (16) in a strong sense.

The second simplifying feature of the toric setting occurs on the quantum level. The Bergman geodesic is obtained by applying the $\mathrm{FS}_{k}$ map to the one-parameter subgroup $e^{t A_{k}}$. In general, it is difficult to understand what kind of asymptotic behavior is possessed by the operators $e^{t A_{k}}$. But on a toric variety, there exists a natural basis of the space of holomorphic sections $H^{0}\left(M, L^{k}\right)$ furnished by monomial sections $z^{\alpha}$ which are orthogonal with respect to all torus-invariant inner products, and with respect to which all change of basis operators $e^{t A_{k}}$ are diagonal; we refer to Section 2 or to [Shiffman et al. 2004] for background. Hence, we only need to analyze the eigenvalues of $e^{A_{k}}$. The exponents $\alpha$ of the monomials are lattice points $\alpha \in k P$ in the $k$-th dilate of the polytope $P$ corresponding to $M$. The eigenvalues in the toric case are given by

$$
\lambda_{\alpha}:=\frac{1}{2} \log \frac{2_{h_{0}^{k}}(\alpha)}{2_{h_{1}^{k}}(\alpha)},
$$

where $\mathscr{2}_{h_{0}^{k}}(\alpha)$ is a norming constant for a toric inner product. By a norming constant for a toric hermitian inner product $G$ on $H^{0}\left(M, L^{k}\right)$ we mean the associated $L^{2}$ norm-squares of the monomials

$$
\mathscr{2}_{G}(\alpha)=\left\|s_{\alpha}\right\|_{G}^{2} .
$$

In particular, if $h \in \mathscr{H}_{\mathbf{T}^{m}}$, the norming constants for $\operatorname{Hilb}_{k}(h)$ are given by

$$
2_{h^{k}}(\alpha)=\left\|s_{\alpha}\right\|_{h^{k}}^{2}:=\int_{M_{P}}\left|s_{\alpha}(z)\right|_{h^{k}}^{2} d V_{h} .
$$


Thus, an orthonormal basis of $H^{0}\left(M, L^{k}\right)$ with respect to $\operatorname{Hilb}_{k}(h)$ for $h \in \mathscr{H}_{T}$ is given by

$$
\left\{\frac{s_{\alpha}}{\sqrt{2_{h^{k}}(\alpha)}}: \alpha \in k P \cap \mathbb{Z}^{m}\right\}
$$

An equivalent, and in a sense dual (see Section 3), formulation is in terms of the functions

$$
\mathscr{P}_{h^{k}}(\alpha, z):=\frac{\left|s_{\alpha}(z)\right|_{h^{k}}^{2}}{2_{h^{k}}(\alpha)},
$$

and their special values

$$
\mathscr{P}_{h^{k}}(\alpha):=\mathscr{P}_{h^{k}}\left(\alpha, \mu_{h}^{-1}\left(\frac{\alpha}{k}\right)\right)=\frac{\left|s_{\alpha}\left(\mu_{h}^{-1}\left(\frac{\alpha}{k}\right)\right)\right|_{h^{k}}^{2}}{2_{h^{k}}(\alpha)} .
$$

Given toric hermitian metrics $h_{0}, h_{1} \in \mathscr{H}_{\mathbf{T}^{m}}$, the change of basis matrix $e^{A_{k}}=\sigma_{h_{0}, h_{1}, k}$ from the monomial orthonormal basis for $\operatorname{Hilb}_{k}\left(h_{0}\right)$ to that for $\operatorname{Hilb}_{k}\left(h_{1}\right)$ is diagonal, and the eigenvalues are given by

$$
\operatorname{Sp}\left(e^{A_{k}} e^{A_{k}^{*}}\right):=\left\{e^{2 \lambda_{\alpha}(k)}=\frac{2_{h_{0}^{k}}(\alpha)}{2_{h_{1}^{k}}(\alpha)}: \alpha \in k P\right\} .
$$

Hence, for a $\mathscr{P}_{k}$-geodesic, (12) becomes

$$
\varphi_{k}(t, z)=\frac{1}{k} \log Z_{k}(t, z),
$$

where

$$
Z_{k}(t, z)=\sum_{\alpha \in k P \cap \mathbb{Z}^{m}}\left(\frac{\mathscr{2}_{h_{0}^{k}}(\alpha)}{\mathscr{2}_{h_{1}^{k}}(\alpha)}\right)^{t} \frac{\left|s_{\alpha}(z)\right|_{h_{0}^{k}}^{2}}{\mathscr{2}_{h_{0}^{k}}(\alpha)} .
$$

It is interesting to observe that the relative Kähler potential (24) is the logarithm of an exponential sum, hence has the form of a free energy of a statistical mechanical problem with states parametrized by $\alpha \in k P$ and with Boltzmann weights

$$
\left(\frac{2_{h_{0}^{k}}(\alpha)}{2_{h_{1}^{k}}(\alpha)}\right)^{t}
$$

Thus, our goal is to prove that

$$
\frac{1}{k} \log \sum_{\alpha \in k P \cap \mathbb{Z}^{m}}\left(\frac{\mathscr{2}_{h_{0}^{k}}(\alpha)}{\mathscr{2}_{h_{1}^{k}}(\alpha)}\right)^{t} \frac{\left|s_{\alpha}(z)\right|_{h_{0}^{k}}^{2}}{\mathscr{2}_{h_{0}^{k}}(\alpha)} \rightarrow \varphi_{t}(z) \quad \text { in } C^{2}([0,1] \times M) .
$$

1D. Heuristic proof. We next sketch a heuristic proof which makes the pointwise convergence obvious. The first step is to obtain good asymptotics of the norming constants (20). As in [Song and Zelditch 2007a], they may be expressed in terms of the symplectic potential by

$$
2_{h^{k}}(\alpha)=\int_{P} e^{-k\left(u_{\varphi}(x)+\left\langle(\alpha / k)-x, \nabla u_{\varphi}(x)\right\rangle\right)} d x
$$


As $k$ tends to $\infty$ the integral is dominated by the unique point $x=\alpha / k$, where the "phase function" is maximized. The Hessian is always nondegenerate and by complex stationary phase we obtain the asymptotics

$$
2_{h^{k}}\left(\alpha_{k}\right) \sim k^{-m / 2} e^{2 k u_{\varphi}(\alpha)} .
$$

The complex stationary phase (or steepest descent) method does not apply near the boundary $\partial P$, causing serious complications, but in this heuristic sketch we ignore this aspect.

If we then replace each term in $Z_{k}$ by its asymptotics, we obtain

$$
\varphi_{k}\left(t, e^{\rho / 2}\right) \sim \frac{1}{k} \log \sum_{\alpha \in P \cap(1 / k) \mathbb{Z}^{m}} e^{2 k\left(u_{0}(\alpha)+t\left(u_{1}(\alpha)-u_{0}(\alpha)\right)+\langle\rho, \alpha\rangle\right)} .
$$

The exponent $\left(u_{0}(\alpha)+t\left(u_{1}(\alpha)-u_{0}(\alpha)\right)+\langle\rho, \alpha\rangle\right)$ is convex and therefore has a unique minimum point. This suggests applying a discrete analogue of complex stationary phase to the sum (28), a DedekindRiemann sum which is asymptotic to the integral

$$
\int_{P} e^{2 k\left(u_{0}(\alpha)+t\left(u_{1}(\alpha)-u_{0}(\alpha)\right)+\langle\rho, \alpha\rangle\right)} d \alpha .
$$

Taking $\frac{1}{k}$ times the log of the integral and applying complex stationary phase gives the asymptote

$$
\max _{\alpha \in P}\left\{u_{0}(\alpha)+t\left(u_{1}(\alpha)-u_{0}(\alpha)\right)+\langle\rho, \alpha\rangle\right\} .
$$

But this is the Legendre transform of the ray of symplectic potentials

$$
u_{\varphi_{0}}(\alpha)+t\left(u_{\varphi_{1}}(\alpha)-u_{\varphi_{0}}(\alpha)\right)
$$

and thus is the Monge-Ampère geodesic.

This is the core idea of the proof. We now give the rigorous version.

1E. Outline of the rigorous proof. The main difficulty in the proof of Theorem 1.1 is that the norms have very different asymptotic regimes according to the position of the normalized lattice point $\alpha / k$ relative to the boundary $\partial P$ of the polytope. Even in the simplest case of $\mathbb{C P}^{m}$, the different positions correspond to the regimes of the central limit theorem, large deviations theorems and Poisson law of rare events for multinomial coefficients. In determining the asymptotics of (24), we face the difficulty that these Boltzmann weights might be exponentially growing or decaying in $k$ as $k \rightarrow \infty$.

To simplify the comparison between the Bergman and Monge-Ampère geodesics, we take advantage of the explicit solution (17) of geodesic equation to rewrite $Z_{k}(t, z)$ in the form

$$
e^{-k \varphi_{t}(z)} Z_{k}(t, z)=\sum_{\alpha \in k P \cap \mathbb{Z}^{m}} \mathscr{R}_{k}(t, \alpha) \frac{\left|s_{\alpha}(z)\right|_{h_{t}^{k}}^{2}}{\mathscr{2}_{h_{t}^{k}}(\alpha)}=\sum_{\alpha \in k P \cap \mathbb{Z}^{m}} \mathscr{R}_{k}(t, \alpha) \mathscr{P}_{h_{t}^{k}}(\alpha, z),
$$

where as usual $h_{t}=e^{-\varphi_{t}} h_{0}$ (with $\varphi_{t}$ as in (17)), and where

$$
\mathscr{R}_{k}(t, \alpha):=\frac{\mathscr{2}_{h_{t}^{k}}(\alpha)}{\left(\mathscr{2}_{h_{0}^{k}}(\alpha)\right)^{1-t}\left(2_{h_{1}^{k}}(\alpha)\right)^{t}} .
$$


One of the key ideas is that $\mathscr{T}_{k}(t, \alpha)$ has at least one order a semiclassical symbol in $k$, that is, it has at least to some extent an asymptotic expansion in powers of $k$. Once this is established, it is possible to prove that

$$
\frac{1}{k} \log \sum_{\alpha \in k P \cap \mathbb{Z}^{m}} \mathscr{R}_{k}(t, \alpha) \mathscr{P}_{h_{t}^{k}}(\alpha, z) \rightarrow 0
$$

in the $C^{2}$-topology on $[0,1] \times M$.

The proof of Theorem 1.1 consists of four main ingredients:

- the Localization Lemma 1.2, which states that the sum over $\alpha$ localizes to a ball of radius $O\left(k^{-1 / 2+\delta}\right)$ around the point $\mu_{h_{t}}(z)$. Here and hereafter, $\delta$ can be taken to be any sufficiently small positive constant;

- Bergman/Szegó asymptotics (see Section 4B), which allow one to make comparisons between the sum in $Z_{k}$ and sums with known asymptotics;

- the Regularity Lemma 1.3, which states that the summands $\mathscr{R}_{k}(t, \alpha)$ one is averaging have sufficiently smooth asymptotics as $k \rightarrow \infty$, allowing one to Taylor expand to order at least one around the point $\mu_{h_{t}}(z)$;

- joint asymptotics of the Fourier coefficients (21) and particularly their special values $\mathscr{P}_{h^{k}}(\alpha)$ in the parameters $k$ and distance to $\partial P$ (see Proposition 6.1). We use a complex stationary phase method in the "interior region" far from $\partial P$ and local Bargmann-Fock models near $\partial P$.

The localization lemma is needed not just for $\mathscr{R}_{k}(t, \alpha)$ but also for summands which arise from differentiation with respect to $(t, z)$.

Lemma 1.2 (localization of sums). Let $B_{k}(t, \alpha): \mathbb{Z}^{m} \cap k P \rightarrow \mathbb{C}$ be a family of lattice point functions satisfying $\left|B_{k}(t, \alpha)\right| \leq C_{0} k^{M}$ for some $C_{0}, M \geq 0$. Then, there exists $C>0$ so that for any $\delta>0$,

$$
\sum_{\alpha \in k P \cap \mathbb{Z}^{m}} B_{k}(t, \alpha) \frac{\left|s_{\alpha}(z)\right|_{h_{t}^{k}}^{2}}{2_{h_{t}^{k}}(\alpha)}=\sum_{\alpha:\left|\frac{\alpha}{k}-\mu_{h_{t}}(z)\right| \leq k^{-1 / 2+\delta}} B_{k}(t, \alpha) \frac{\left|s_{\alpha}(z)\right|_{h_{t}^{k}}^{2}}{2_{h_{t}^{k}}(\alpha)}+O_{\delta}\left(k^{-C}\right) .
$$

The proof is based on integration by parts. One could localize to the smaller scale

$$
\left|\frac{\alpha}{k}-\mu_{h_{t}}(z)\right| \leq C \frac{\log k}{\sqrt{k}},
$$

but then the argument only brings errors of the order $(\log k)^{-M}$ for all $M$ and that complicates later applications.

The regularity lemma concerns the behavior of the Fourier multiplier $R_{k}(t, \alpha)(30)$. The sum (25) formally resembles the Berezin covariant symbol of a Toeplitz Fourier multiplier, that is, the restriction to the diagonal of the Schwartz kernel of the operator; we refer to [Shiffman et al. 2003; Zelditch 2009] for discussion of such Toeplitz Fourier multipliers operators on toric varieties and their Berezin symbols. However, the resemblance is a priori just formal - it is not obvious that $R_{k}(t, \alpha)$ has asymptotics in $k$. As mentioned above, the nature of the asymptotics is most difficult near $\partial P$; it is not obvious that smooth convergence holds along $\mathscr{D}$, the divisor at infinity. 
Definition. We define the metric volume ratio to be the function on $[0,1] \times P$ defined by

$$
\mathscr{R}_{\infty}(t, x):=\left(\frac{\operatorname{det} \nabla^{2} u_{t}(x)}{\left(\operatorname{det} \nabla^{2} u_{0}(x)\right)^{1-t}\left(\operatorname{det} \nabla^{2} u_{1}(x)\right)^{t}}\right)^{1 / 2} .
$$

The purpose of introducing $R_{k}(t, \alpha)$ is explained by the following result.

Lemma 1.3 (regularity). The volume ratio $\mathscr{R}_{\infty}(t, x) \in C^{\infty}([0,1] \times P)$. Further, for $0 \leq j \leq 2$,

$$
\left(\frac{\partial}{\partial t}\right)^{j} \mathscr{R}_{k}(t, \alpha)=\left(\frac{\partial}{\partial t}\right)^{j} \mathscr{R}_{\infty}\left(t, \frac{\alpha}{k}\right)+O\left(k^{-1 / 3}\right),
$$

where the $O$ symbol is uniform in $(t, \alpha)$.

This lemma is the subtlest part of the analysis. If the $\mathscr{R}_{k}$ function were replaced by a fixed function $f(x)$ evaluated at $\alpha / k$ then the convergence problem reduces to generalizations of convergence of Bernstein polynomial approximations to smooth functions [Zelditch 2009], and only requires now standard Bergman kernel asymptotics. However, the actual $R_{k}(t, \alpha)$ do not a priori have this form, and much more is required for their analysis than asymptotics (on and off diagonal) of Bergman kernels. The analysis uses a mixture of complex stationary phase arguments in directions where $\alpha / k$ is not too close to $\partial P$, while for directions close to $\partial P$ we use an approximation by the "linear" Bargmann-Fock model (see Section 2F and Section 6D).

The somewhat unexpected $k^{-1 / 3}$ remainder estimate has its origin in this mixture of complex stationary phase and Bargmann-Fock asymptotics. Both methods are valid for $k$ satisfying

$$
\frac{C \log k}{k} \leq \delta_{k} \leq C^{\prime} \frac{1}{\sqrt{k} \log k} .
$$

In this region, the stationary phase remainder is of order $\left(k \delta_{k}\right)^{-1}$ while the Bargmann-Fock remainder is of order $k \delta_{k}^{2}$; the two remainders agree when $\delta_{k}=k^{-2 / 3}$, and then the remainder is $O\left(k^{-1 / 3}\right)$. For smaller $\delta_{k}$ the Bargmann-Fock approximation is more accurate and for larger $\delta_{k}$ the stationary phase approximation is more accurate. This matter is discussed in detail in Section 6D.

The rest of the proof of the $C^{2}$-convergence may be roughly outlined as follows: We calculate two logarithmic derivatives of $e^{-k \varphi_{t}(z)} Z_{k}(t, z)$ of (29) with respect to $(t, \rho)$. Using the Localization Lemma 1.2 we can drop the terms in the resulting sums corresponding to $\alpha$ for which $\left|\frac{\alpha}{k}-\mu_{h_{t}}(z)\right|>k^{-1 / 2+\delta}$. In the remaining terms we use the Regularity Lemma 1.3 to approximate the summands by their Taylor expansions to order one around $\mu_{h_{t}}(z)$. This reduces the expressions to derivatives of the diagonal Szegó kernel

$$
\Pi_{h_{t}^{k}}(z, z)=\sum_{\alpha \in k P \cap \mathbb{Z}^{m}} \frac{\left|s_{\alpha}(z)\right|_{h_{t}^{k}}^{2}}{2_{h_{t}^{k}}(\alpha)}
$$

for the metric $h_{t}^{k}$ on $H^{0}\left(M, L^{k}\right)$ induced by Monge-Ampère geodesic $h_{t}$. Here, we use the smoothness of $h_{t}$. The known asymptotic expansion of this kernel (Section 4B) implies the $C^{2}$-convergence of $e^{k \varphi_{t}(z)} Z_{k}(t, z)$.

As indicated in this sketch, the key problem is to analyze the joint asymptotics of norming constants $2_{h}^{k}(\alpha)$ and the dual constants $\mathscr{P}_{h^{k}}(\alpha)(22)$ in $(k, \alpha)$. Norming constants are a complete set of invariants of toric Kähler metrics. Initial results (but not joint asymptotics in the boundary regime) were obtained in [Shiffman et al. 2004]; norms are also an important component of Donaldson's numerical analysis 
of canonical metrics on toric varieties [Donaldson 2005]. Song and Zelditch [2007a] studied the joint asymptotics of $2_{h}^{k}(\alpha)$ up to the boundary of the polytope $[0,1]$ associated to $\mathbb{C P}^{1}$. In this article, we emphasize the dual constants (22).

1F. Bergman approximation and complexification. Having described our methods and results, we return to the discussion of their relation to Kähler quantization and to the obstacles in complexifying $\operatorname{Diff}_{\omega_{0}}(M)$. Further discussion is given in [Rubinstein and Zelditch $\geq 2010 \mathrm{~b}$ ].

We may distinguish two intuitive ideas as to the nature of Monge-Ampère geodesics. The first heuristic idea, due to Semmes [1992] and Donaldson [2001], is to view HCMA (homogeneous complex Monge-Ampère) geodesics as one-parameter subgroups of $G_{\mathbb{C}}$, where $G=\operatorname{SDiff}_{\omega_{0}}(M)$. One-parameter subgroups of $\operatorname{SDiff}_{\omega_{0}}(M)$ are defined by Hamiltonian flows of initial Hamiltonians $\dot{\varphi}_{0}$ with respect to $\omega_{0}$. A complexified one-parameter subgroup is the analytic continuation in time of such a Hamiltonian flow [Semmes 1992; Donaldson 2001]. This idea is heuristic inasmuch as Hamiltonian flows need not possess analytic continuations in time; moreover, no genuine complexification of $\operatorname{SDiff}_{\omega_{0}}(M)$ exists.

The second intuitive idea, backed up by [Phong and Sturm 2006] and this article, is to view HCMA geodesics as classical limits of $\mathscr{P}_{k}$ geodesics. The latter have a very simple extrinsic interpretation as one-parameter motions $e^{t A_{k}} \underline{s}_{\underline{s}}(M)$ of a holomorphic embedding $l_{\underline{s}}: M \rightarrow \mathbb{C P}^{d_{k}}$. But the passage to the classical limit is quite nonstandard from the point of view of Kähler quantization. The problem is that the approximating one parameter subgroups $e^{t A_{k}}$ of operators on $H^{0}\left(M, L^{k}\right)$, which change an orthonormal basis for an initial inner product to a path of orthonormal bases for the geodesic of inner products, are not a priori complex Fourier integral operators or any known kind of quantization of classical dynamics.

The heuristic view taken in this article and series is that $e^{t A_{k}}$ should be approximately the analytic continuation of the Kähler quantization of a classical Hamiltonian flow. To explain this, let us recall the basic ideas of Kähler quantization.

Traditionally, Kähler quantization refers to the quantization of a polarized Kähler manifold $(M, \omega, L)$ by Hilbert spaces $H^{0}\left(M, L^{k}\right)$ of holomorphic sections of high powers of a holomorphic line bundle $L \rightarrow M$ with Chern class $c_{1}(L)=[\omega]$. The Kähler form determines a hermitian metric $h$ such that $\operatorname{Ric}(h)=\omega$. The hermitian metric induces inner products $\operatorname{Hilb}_{k}(h)$ on $H^{0}\left(M, L^{k}\right)$. In this quantization theory, functions $H$ on $M$ are quantized as hermitian (Toeplitz) operators $\hat{H}:=\Pi_{h^{k}} H \Pi_{h^{k}}$ on $H^{0}\left(M, L^{k}\right)$, and canonical transformations of $(M, \omega)$ are quantized as unitary operators on $H^{0}\left(M, L^{k}\right)$. Quantum dynamics is given by unitary groups $e^{i t k \hat{H}}$ (see [Berman et al. 2008; Boutet de Monvel and Sjöstrand 1976; Zelditch 1998] for references).

In the case of Bergman geodesics with fixed endpoints, $H$ should be $\dot{\varphi}_{0}$, the initial tangent vector to the HCMA geodesic with the fixed endpoints. The quantization of the Hamiltonian flow of $\dot{\varphi}$ should then be $e^{i t k \hat{H}}$ and its analytic continuation should be $e^{t k \hat{H}}$. The change of basis operator $e^{t A_{k}}$ should then be approximately the same as $e^{t k \hat{H}}$. But proving this and taking the classical limit is necessarily nonstandard when the classical analytic continuation of the Hamiltonian flow of $\dot{\varphi}$ does not exist. Moreover, we only know that $\dot{\varphi} \in C^{0,1}$.

This picture of the Bergman approximation to HCMA geodesics is validated in this article in the case of the Dirichlet problem on projective toric Kähler manifolds. Y. Rubinstein and the second author have also verified for the initial value problem on toric Kähler manifolds [Rubinstein and Zelditch $\geq 2010 \mathrm{~b}$ ]. The same authors are currently investigating it for general Kähler metrics on Riemann surfaces. 
1G. Final remarks and further results and problems. An obvious question within the toric setting is whether $\varphi_{k}(t) \rightarrow \varphi_{t}$ in a stronger topology than $C^{2}$ on a toric variety. It seems possible that the methods of this paper could be extended to $C^{k}$-convergence. The methods of this paper easily imply $C^{k}$-convergence for all $k$ away from $\partial P$ or equivalently the divisor at infinity, but the degree of convergence along this set has yet to be investigated. As mentioned above, we do not see why $\varphi_{k}$ should have an asymptotic expansion in $k$, but this aspect may deserve further exploration. We also mention that our methods can be extended to prove $C^{2}$-convergence of Berndtsson's approximations in [Berndtsson 2009].

In subsequent articles on the toric case, we build on the methods introduced here to prove convergence theorems. In [Song and Zelditch 2007b], we develop the methods of this article to prove that the geodesic rays constructed in [Phong and Sturm 2007] from test configurations are $C^{1,1}$ and no better on a toric variety. Test configuration geodesic rays are solutions of a kind of initial value problem; we refer to [Phong and Sturm 2007; Song and Zelditch 2007b] for the definitions and results. For test configuration geodesics, the analogue of $\mathscr{R}_{k}$ is not even smooth in $t$. The smooth initial value problem is studied in [Rubinstein and Zelditch $\geq 2010 \mathrm{~b}$ ]. In a different direction, a $C^{2}$ convergence result has been proved for completely general harmonic maps of Riemannian manifolds with boundary into toric varieties [Rubinstein 2008; Rubinstein and Zelditch 2010]. This includes the Wess-Zumino-Witten model, where the manifold is a Riemann surface with boundary.

We believe that the techniques of this paper extend to other settings with a high degree of symmetry of the kinds discussed in [Donaldson 2008]. Recently, Feng [2009] adapted our methods to "toric" metrics on abelian varieties, that is, metrics which are invariant under a real Lagrangian torus action. Associated to the torus action is a torus-valued moment map. Abelian varieties are simpler than toric varieties in that the image of the moment map is the full torus; that is, there is no boundary to the image. Consequently, Feng [2009] is able to improve Lemma 1.3 and then Theorem 1.1 to give $C^{\infty}$ convergence and complete asymptotics expansions. The general Kähler case involves significant further obstacles. A basic problem in generalizing the results is to construct a useful localized basis of sections on a general $(M, \omega)$. In the toric case, we use the basis of $\mathbf{T}^{m}$-invariant states $\hat{s}_{\alpha}=z^{\alpha}$, which "localize" on the so-called "BohrSommerfeld tori", that is, the inverse images $\mu^{-1}\left(\frac{\alpha}{k}\right)$ of lattice points under the moment map $\mu$. Such Bohr-Sommerfeld states also exist on any Riemann surface; in subsequent work, we hope to relate them to the convergence problem for HCMA geodesics on Riemann surfaces.

We briefly speculate on the higher-dimensional general Kähler case. There are a number of plausible substitutes for the Bohr-Sommerfeld basis on a general Kähler manifold. A rather traditional one is to study the asymptotics of $e^{A_{k}}$ on a basis of coherent states $\Phi_{h^{k}}^{w}$. Here,

$$
\Phi_{h^{k}}^{w}(z)=\frac{\Pi_{h^{k}}(z, w)}{\sqrt{\Pi_{h^{k}}(w, w)}}
$$

are $L^{2}$ normalized Szegó kernels pinned down in the second argument. Intuitively, $\Phi_{h^{k}}^{w}$ is like a gaussian bump centered at $w$ with shape determined by the metric $h$. It is thus more localized than the monomials $z^{\alpha}$, which are only gaussian transverse to the tori. Under the change of basis operators $e^{t A_{k}}$, both the center and shape should change. Like the monomials $z^{\alpha}$, coherent states have some degree of orthogonality. There are in addition other well localized bases depending on the Kähler metric which may be used in the analysis. 
Our main result (Theorem 1.1) may be viewed heuristically as showing that as $k \rightarrow \infty$ the change of basis operators $e^{t A_{k}}$ tend to a path $f_{t}$ of diffeomorphisms changing the initial Kähler metric $\omega_{0}$ into the metric $\omega_{t}$ along the Monge-Ampère geodesic. This suggests that

$$
e^{t A_{k}} \Phi_{h^{k}}^{w} \sim \Phi_{h_{t}^{k}}^{f_{t}(w)}
$$

where $h_{t}$ is the Monge-Ampère geodesic and $f_{t}$ is the Moser path of diffeomorphisms such that $f_{t}^{*} \omega_{0}=$ $\omega_{t}$. We leave the exact degree of asymptotic similarity vague at this time since even the regularity of the Moser path is currently an open problem.

\section{Background on toric varieties}

In this section, we review the necessary background on toric Kähler manifolds. In addition to standard material on Kähler and symplectic potentials, moment maps and polytopes, we also present some rather nonstandard material on almost analytic extensions of Kähler potentials and moment maps that are needed later on. We also give a simple proof that the Legendre transform from Kähler potentials to symplectic potentials linearizes the Monge-Ampère equation.

Let $M$ be a complex manifold. We use the standard notation

$$
\partial=\frac{\partial}{\partial z}=\frac{1}{2}\left(\frac{\partial}{\partial x}-i \frac{\partial}{\partial y}\right), \quad \bar{\partial}=\frac{\partial}{\partial \bar{z}}=\frac{1}{2}\left(\frac{\partial}{\partial x}+i \frac{\partial}{\partial y}\right), \quad d=\partial+\bar{\partial}, \quad d^{c}:=\frac{i}{4 \pi}(\bar{\partial}-\partial), \quad d d^{c}=\frac{i}{2 \pi} \partial \bar{\partial} .
$$

The last three are real operators.

Let $L \rightarrow M$ be a holomorphic line bundle. The Chern form of a hermitian metric $h$ on $L$ is defined by

$$
c_{1}(h)=\omega_{h}:=-\frac{\sqrt{-1}}{2 \pi} \partial \bar{\partial} \log \left\|e_{L}\right\|_{h}^{2},
$$

where $e_{L}$ denotes a local holomorphic frame (= nonvanishing section) of $L$ over an open set $U \subset M$, and $\left\|e_{L}\right\|_{h}=h\left(e_{L}, e_{L}\right)^{1 / 2}$ denotes the $h$-norm of $e_{L}$. We say that $(L, h)$ is positive if the (real) 2-form $\omega_{h}$ is a positive $(1,1)$ form, that is, defines a Kähler metric. We write $\left\|e_{L}(z)\right\|_{h}^{2}=e^{-\varphi}$ or locally $h=e^{-\varphi}$, and then refer to $\varphi$ as the Kähler potential of $\omega_{h}$ in $U$. In this notation,

$$
\omega_{h}=\frac{\sqrt{-1}}{2 \pi} \partial \bar{\partial} \varphi=d d^{c} \varphi .
$$

If we fix a hermitian metric $h_{0}$ and let $h=e^{-\varphi} h_{0}$, and put $\omega_{0}=\omega_{h_{0}}$, then

$$
\omega_{h}=\omega_{0}+d d^{c} \varphi .
$$

The metric $h$ induces hermitian metrics $h^{k}$ on $L^{k}=L \otimes \cdots \otimes L$ given by $\left\|s^{\otimes k}\right\|_{h_{N}}=\|s\|_{h}^{k}$.

We now specialize to toric Kähler manifolds; for background, we refer to [Abreu 2003; Donaldson 2002; Guan 1999; Shiffman et al. 2004]. A toric Kähler manifold is a Kähler manifold $(M, J, \omega)$ on which the complex torus $\left(\mathbb{C}^{*}\right)^{m}$ acts holomorphically with an open orbit $M^{o}$. Choosing a basepoint $m_{0}$ on the open orbit identifies $M^{o} \equiv\left(\mathbb{C}^{*}\right)^{m}$ and give the point $z=e^{\rho / 2+i \varphi} m_{0}$ the holomorphic coordinates

$$
z=e^{\rho / 2+i \varphi} \in\left(\mathbb{C}^{*}\right)^{m}, \quad \rho, \varphi \in \mathbb{R}^{m} .
$$


The real torus $\mathbf{T}^{m} \subset\left(\mathbb{C}^{*}\right)^{m}$ acts in a Hamiltonian fashion with respect to $\omega$. Its moment map

$$
\mu=\mu_{\omega}: M \rightarrow P \subset \mathbf{t}^{*} \simeq \mathbb{R}^{m}
$$

(where $\mathbf{t}$ is the Lie algebra of $\mathbf{T}^{m}$ ) with respect to $\omega$ defines a singular torus fibration over a convex lattice polytope $P$; as in the introduction, $P$ is understood to be the closed polytope. We recall that the moment map of a Hamiltonian torus action with respect to a symplectic form $\omega$ is the map $\mu_{\omega}: M \rightarrow \mathbf{t}^{*}$ defined by $d\left\langle\mu_{\omega}(z), \xi\right\rangle=l_{\xi \#} \omega$, where $\xi^{\#}$ is the vector field on $M$ induced by the vector $\xi \in \mathbf{t}$. Over the open orbit one thus has a symplectic identification

$$
\mu: M^{o} \simeq P^{o} \times \mathbf{T}^{m}
$$

We let $x$ denote the Euclidean coordinates on $P$. The components $\left(I_{1}, \ldots, I_{m}\right)$ of the moment map are called action variables for the torus action. The symplectically dual variables on $\mathbf{T}^{m}$ are called the angle variables. Given a basis of $\mathbf{t}$ or equivalently of the action variables, we denote by $\left\{\partial / \partial \theta_{j}\right\}$ the corresponding generators (Hamiltonian vector fields) of the $\mathbf{T}^{m}$ action. Under the complex structure $J$, we also obtain generators $\partial / \partial \rho_{j}$ of the $\mathbb{R}_{+}^{m}$ action.

The action variables are globally defined smooth functions but fail to be coordinates at points where the generators of the $\mathbf{T}^{m}$ action vanish. We denote the set of such points by $\mathscr{D}$ and refer to it as the divisor at infinity. If $p \in \mathscr{D}$ and $\mathbf{T}_{p}^{m}$ denotes the isotropy group of $p$, then the generating vector fields of $\mathbf{T}_{p}^{m}$ become linearly dependent at $P$. Since we are proving $C^{2}$ estimates, we need to replace them near points of $\mathscr{D}$ by vector fields with norms bounded below. We discuss good choices of coordinates near points of $\mathscr{D}$ below.

We assume $M$ is smooth and that $P$ is a Delzant polytope. It is defined by a set of linear inequalities

$$
\ell_{r}(x):=\left\langle x, v_{r}\right\rangle-\lambda_{r} \geq 0, \quad r=1, \ldots, d,
$$

where $v_{r}$ is a primitive element of the lattice and inward-pointing normal to the $r$-th $(m-1)$-dimensional facet $F_{r}=\left\{\ell_{r}=0\right\}$ of $P$. We recall that a facet is a highest-dimensional face of a polytope. The inverse image $\mu^{-1}(\partial P)$ of the boundary of $P$ is the divisor at infinity $\mathscr{D} \subset M$. For $x \in \partial P$ we denote by

$$
\mathscr{F}(x)=\left\{r: \ell_{r}(x)=0\right\}
$$

the set of facets containing $x$. To measure when $x \in P$ is near the boundary we further define

$$
\mathscr{F}_{\epsilon}(x)=\left\{r:\left|\ell_{r}(x)\right|<\epsilon\right\} .
$$

The simplest toric varieties are linear Kähler manifolds $(V, \omega)$ carrying a linear holomorphic torus action. They provide local models near a corner of $P$ or equivalently near a fixed point of the $\mathbf{T}^{m}$ action. As discussed in [Guillemin and Sternberg 1982; Lerman and Tolman 1997], a linear symplectic torus action is determined by a choice of $m$ elements $\beta_{j}$ of the weight lattice of the Lie algebra of the torus. The vector space then decomposes $(V, \omega)=\bigoplus\left(V_{i}, \omega_{i}\right)$ of orthogonal symplectic subspaces so that the moment map has the form

$$
\mu_{B F}\left(v_{1}, \ldots, v_{m}\right)=\sum\left|v_{j}\right|^{2} \beta_{j} .
$$

The image of the moment map is the orthant $\mathbb{R}_{+}^{m}$. This provides a useful local model at corners. We refer to these as Bargmann-Fock models; they play a fundamental role in this article (see Section $2 \mathrm{~F}$ ). 
2A. Slice-orbit coordinates. We will also need local models at points near codimension $r$ faces, and therefore supplement the coordinates (36) on the open orbit with holomorphic coordinates valid in neighborhoods of points of $\mathscr{D}$. An atlas of coordinate charts for $M$ generalizing the usual affine charts of $\mathbb{C P}^{m}$ is given in [Shiffman et al. 2004, Section 3.2], and we briefly recall the definitions. For each vertex $v_{0} \in P$, we define the chart $U_{v_{0}}$ by

$$
U_{v_{0}}:=\left\{z \in M_{P}: \chi_{v_{0}}(z) \neq 0\right\}
$$

where

$$
\chi_{\alpha}(z)=z^{\alpha}=z_{1}^{\alpha_{1}} \cdots z_{m}^{\alpha_{m}} .
$$

Throughout the article we use standard multiindex notation, and put $|\alpha|=\alpha_{1}+\cdots+\alpha_{m}$. Since $P$ is Delzant, we can choose lattice points $\alpha^{1}, \ldots, \alpha^{m}$ in $P$ such that each $\alpha^{j}$ is in an edge incident to the vertex $v_{0}$, and the vectors $v^{j}:=\alpha^{j}-v_{0}$ form a basis of $\mathbb{Z}^{m}$. We define

$$
\eta:\left(\mathbb{C}^{*}\right)^{m} \rightarrow\left(\mathbb{C}^{*}\right)^{m}, \quad \eta(z)=\eta_{j}(z):=\left(z^{v^{1}}, \ldots, z^{v^{m}}\right) .
$$

The map $\eta$ is a $\mathbf{T}^{m}$-equivariant biholomorphism with inverse

$$
z:\left(\mathbb{C}^{*}\right)^{m} \rightarrow\left(\mathbb{C}^{*}\right)^{m}, \quad z(\eta)=\left(\eta^{\Gamma e^{1}}, \ldots, \eta^{\Gamma e^{m}}\right),
$$

where $e^{j}$ is the standard basis for $\mathbb{C}^{m}$, and $\Gamma$ is an $m \times m$-matrix with det $\Gamma= \pm 1$ and integer coefficients defined by

$$
\Gamma v^{j}=e^{j}, \quad v^{j}=\alpha^{j}-v_{0} .
$$

The corner of $P$ at $v_{0}$ is transformed to the standard corner of the orthant $\mathbb{R}_{+}^{m}$ by the affine linear transformation

$$
\tilde{\Gamma}: \mathbb{R}^{m} \ni u \rightarrow \Gamma u-\Gamma v_{0} \in \mathbb{R}^{m},
$$

which preserves $\mathbb{Z}^{m}$, carries $P$ to a polytope $Q_{v_{0}} \subset\left\{x \in \mathbb{R}^{m}: x_{j} \geq 0\right\}$ and carries the facets $F_{j}$ incident at $v_{0}$ to the coordinate hyperplanes $=\left\{x \in Q_{v_{0}} ; x_{j}=0\right\}$. The map $\eta$ extends to a homeomorphism

$$
\eta: U_{v_{0}} \rightarrow \mathbb{C}^{m}, \quad \eta\left(z_{0}\right)=0
$$

where $z_{0}$ is the fixed point corresponding to $v_{0}$. By this homeomorphism, the set $\mu_{P}^{-1}\left(\bar{F}_{j}\right)$ corresponds to the set $\left\{\eta \in \mathbb{C}^{m}: \eta_{j}=0\right\}$. If $\bar{F}$ be a closed face with $\operatorname{dim} F=m-r$ which contains $v_{0}$, then there are facets $F_{i_{1}}, \ldots, F_{i_{r}}$ incident at $v_{0}$ such that $\bar{F}=\bar{F}_{i_{1}} \cap \cdots \cap \bar{F}_{i_{r}}$. The subvariety $\mu_{P}^{-1}(\bar{F})$ corresponding $\bar{F}$ is expressed by

$$
\mu_{P}^{-1}(\bar{F}) \cap U_{v_{0}}=\left\{\eta \in \mathbb{C}^{m}: \eta_{i_{j}}=0, j=1, \ldots, r\right\} .
$$

When working near a point of $\mu_{P}^{-1}(\bar{F})$, we simplify notation by writing

$$
\eta=\left(\eta^{\prime}, \eta^{\prime \prime}\right) \in \mathbb{C}^{m}=\mathbb{C}^{r} \times \mathbb{C}^{m-r},
$$

where $\eta^{\prime}=\left(\eta_{i_{j}}\right)$ as in (45) and where $\eta^{\prime \prime}$ are the remaining $\eta_{j}$ 's, so that $\left(0, \eta^{\prime \prime}\right)$ is a local coordinate of the submanifold $\mu_{P}^{-1}(\bar{F})$. When the point $\left(0, \eta^{\prime \prime}\right)$ lies in the open orbit of $\mu_{P}^{-1}(\bar{F})$, we often write $\eta^{\prime \prime}=e^{i \theta^{\prime \prime}+\rho^{\prime \prime} / 2}$. In practice, we simplify notation by tacitly treating the corner at $v_{0}$ as if it were the standard corner of $\mathbb{R}_{+}^{m}$, omit mention of $\Gamma$ and always use $\left(z^{\prime}, z^{\prime \prime}\right)$ instead of $\eta$. It is straightforward to rewrite all the expressions we use in terms of the more careful coordinate charts just mentioned. 
These coordinates may be described more geometrically as slice-orbit coordinates. Set $P_{0} \in \mu_{P}^{-1}(\bar{F})$ and let $\left(\mathbb{C}^{*}\right)_{P_{0}}^{m}$ denote its stabilizer (isotropy subgroup). Then there always exists a local slice at $P_{0}$, that is, a local analytic subspace $S \subset M$ containing $P_{0}$, invariant under $\left(\mathbb{C}^{*}\right)_{P_{0}}^{m}$, and such that the natural $\left(\mathbb{C}^{*}\right)^{m}$-equivariant map of the normal bundle of the orbit $\left(\mathbb{C}^{*}\right)^{m} \cdot P_{0}$, namely

$$
[\zeta, P] \in\left(\mathbb{C}^{*}\right)^{m} \times\left(\mathbb{C}^{*}\right)_{z}^{m} S \mapsto \zeta P \in M,
$$

is a biholomorphism onto $\left(\mathbb{C}^{*}\right)^{m} \cdot S$. The terminology is taken from [Sjamaar 1995, Theorem 1.23]. The slice $S$ can be taken to be the image of a ball in the hermitian normal space $T_{P_{0}}\left(\left(\mathbb{C}^{*}\right)^{m} P_{0}\right)^{\perp}$ to the orbit under any local holomorphic embedding $w: T_{P_{0}}\left(\left(\mathbb{C}^{*}\right)^{m} P_{0}\right)^{\perp} \rightarrow M$ with $w\left(P_{0}\right)=P_{0}$ and $d w_{P_{0}}=$ Id. The affine coordinates $\eta^{\prime \prime}$ above define the slice $S=\eta^{-1}\left\{\left(z^{\prime}, z^{\prime \prime}\left(P_{0}\right)\right): z^{\prime} \in\left(\mathbb{C}^{*}\right)^{r}\right\}$. The local orbit-slice coordinates are then defined by

$$
P=\left(z^{\prime}, e^{i \theta^{\prime \prime}+\rho^{\prime \prime} / 2}\right) \Longleftrightarrow \eta(P)=e^{i \theta^{\prime \prime}+\rho^{\prime \prime} / 2}\left(z^{\prime}, 0\right),
$$

where $\left(z^{\prime}, 0\right) \in S$ is the point on the slice with affine holomorphic coordinates $z^{\prime}=\left(\eta^{\prime}\right)$.

As will be seen below, toric functions are smooth functions of the variables $e^{\rho_{j}}$ away from $\mathscr{D}$, and of the variables $\left|z_{j}\right|^{2}$ at points near $\mathscr{D}$. We introduce the following polar coordinates centered at a point $P \in \mathscr{D}$ :

$$
r_{j}:=\left|z_{j}\right|=e^{\rho_{j} / 2} .
$$

They are polar coordinates along the slice. The gradient vector field of $r_{j}$ is denoted $\partial / \partial r_{j}$. As with polar vector fields, it is not well-defined at $r_{j}=0$. But to prove $C^{\ell}$ estimates of functions which are smooth functions of $r_{j}^{2}$ it is sufficient to prove $C^{\ell}$ estimates with respect to the vector fields $\partial / \partial r_{j}$ or $\partial / \partial\left(r_{j}^{2}\right)$.

2B. Kähler potential in the open orbit and symplectic potential. Now consider the Kähler metrics $\omega$ in $\mathscr{H}($ see (1)). We recall that on any simply connected open set, a Kähler metric may be locally expressed as $\omega=2 i \partial \bar{\partial} \varphi$, where $\varphi$ is a locally defined function which is unique up to the addition $\varphi \mapsto \varphi+f(z)+\overline{f(z)}$ of the real part of a holomorphic or antiholomorphic function $f$. Here, $a \in \mathbb{R}$ is a real constant which depends on the choice of coordinates. Thus, a Kähler metric $\omega \in \mathscr{H}$ has a Kähler potential $\varphi$ over the open orbit $M^{o} \subset M$. In fact, there is a canonical choice of the open-orbit Kähler potential once one fixes the image $P$ of the moment map:

$$
\varphi(z)=\log \sum_{\alpha \in P}\left|z^{\alpha}\right|^{2}=\log \sum_{\alpha \in P} e^{\langle\alpha, \rho\rangle} .
$$

Invariance under the real torus action implies that $\varphi$ only depends on the $\rho$-variables, so that we may write it in the form

$$
\varphi(z)=\varphi(\rho)=F\left(e^{\rho}\right) .
$$

The notation $\varphi(z)=\varphi(\rho)$ is an abuse of notation, but is rather standard since [Donaldson 2002]. For instance, the Fubini-Study Kähler potential is $\varphi(z)=\log \left(1+|z|^{2}\right)=\log \left(1+e^{\rho}\right)=F\left(e^{\rho}\right)$. Note that the Kähler potential $\log \left(1+|z|^{2}\right)$ extends to $\mathbb{C}^{m}$ from the open orbit $\left(\mathbb{C}^{*}\right)^{m}$, although the coordinates $(\rho, \theta)$ are only valid on the open orbit. This is a typical situation. 
On the open orbit, we then have

$$
\omega_{\varphi}=\frac{i}{2} \sum_{j, k} \frac{\partial^{2} \varphi(\rho)}{\partial \rho_{k} \partial \rho_{j}} \frac{d z_{j}}{z_{j}} \wedge \frac{d \bar{z}_{k}}{\bar{z}_{k}} .
$$

Positivity of $\omega_{\varphi}$ implies that $\varphi(\rho)=F\left(e^{\rho}\right)$ is a strictly convex function of $\rho \in \mathbb{R}^{n}$. The moment map with respect to $\omega_{\varphi}$ is given on the open orbit by

$$
\mu_{\omega_{\varphi}}\left(z_{1}, \ldots, z_{m}\right)=\nabla_{\rho} \varphi(\rho)=\nabla_{\rho} F\left(e^{\rho_{1}}, \ldots, e^{\rho_{m}}\right), \quad\left(z=e^{\rho / 2+i \theta}\right) .
$$

Here, and henceforth, we subscript moments maps either by the hermitian metric $h$ or by a local Kähler potential $\varphi$. The formula (53) follows from the fact that the generators $\partial / \partial \theta_{j}$ of the $\mathbf{T}^{m}$ actions are Hamiltonian vector fields with respect to $\omega_{\varphi}$ with Hamiltonians $\partial \varphi(\rho) / \partial \rho_{j}$, since

$$
l_{\partial / \partial \theta_{j}} \omega_{\varphi}=d \frac{\partial \varphi}{\partial \rho_{j}} .
$$

The moment map is a homeomorphism from $\rho \in \mathbb{R}^{m}$ to the interior $P^{o}$ of $P$ and extends as a smooth map from $M \rightarrow \bar{P}$ with critical points on the divisor at infinity $\mathscr{D}$. Hence, the Hamiltonians (54) extend to $\mathscr{D}$.

Note that the local Kähler potential on the open orbit is not the same as the global smooth relative Kähler potential in (1) with respect to a background Kähler metric $\omega_{0}$. That is, given a reference metric $\omega_{0}$ with Kähler potential $\varphi_{0}$, it follows by the $\partial \bar{\partial}$ lemma that $\omega=\omega_{0}+d d^{c} \varphi$ with $\varphi \in C^{\infty}(M)$. As discussed in [Donaldson 2002, Proposition 3.1.7], the Kähler potential $\varphi$ on the open orbit defines a singular potential on $M$ which satisfies $d d^{c} \varphi=\omega+H$ where $H$ is a fixed current supported on $\mathscr{D}$. We generally denote Kähler potentials by $\varphi$ and in each context explain which type we mean.

By (52), a $\mathbf{T}^{m}$-invariant Kähler potential defines a real convex function on $\rho \in \mathbb{R}^{m}$. Its Legendre dual is the symplectic potential $u_{\varphi}$ : for $x \in P$ there is a unique $\rho$ such that $\mu_{\varphi}\left(e^{\rho / 2}\right)=\nabla_{\rho} \varphi=x$. Then the Legendre transform is defined to be the convex function

$$
u_{\varphi}(x)=\left\langle x, \rho_{x}\right\rangle-\varphi\left(\rho_{x}\right), \quad e^{\rho_{x} / 2}=\mu_{\varphi}^{-1}(x) \Longleftrightarrow \rho_{x}=2 \log \mu_{\varphi}^{-1}(x)
$$

on $P$. The gradient $\nabla_{x} u_{\varphi}$ is an inverse to $\mu_{\omega_{\varphi}}$ on $M_{\mathbb{R}}$ on the open orbit, or equivalently on $P$, in the sense that $\nabla u_{\varphi}\left(\mu_{\omega_{\varphi}}(z)\right)=z$ as long as $\mu_{\omega_{\varphi}}(z) \notin \partial P$.

The symplectic potential has canonical logarithmic singularities on $\partial P$. According to [Abreu 2003, Proposition 2.8] or [Donaldson 2002, Proposition 3.1.7], there is a one-to-one correspondence between $\mathbf{T}_{\mathbb{R}}^{m}$-invariant Kähler potentials $\psi$ on $M_{P}$ and symplectic potentials $u$ in the class $S$ of continuous convex functions on $\bar{P}$ such that $u-u_{0}$ is smooth on $\bar{P}$ where

$$
u_{0}(x)=\sum_{k} \ell_{k}(x) \log \ell_{k}(x)
$$

Thus, $u_{\varphi}(x)=u_{0}(x)+f_{\varphi}(x)$ where $f_{\varphi} \in C^{\infty}(\bar{P})$. We note that $u_{0}$ and $u_{\varphi}$ are convex, that $u_{0}=0$ on $\partial P$ and hence $u_{\varphi}=f_{\varphi}$ on $\partial P$. By convexity, $\max _{P} u_{0}=0$.

We denote by $G_{\varphi}=\nabla_{x}^{2} u_{\varphi}$ the Hessian of the symplectic potential. It has simple poles on $\partial P$. It follows that $\nabla_{\rho}^{2} \varphi$ has a kernel along $\mathscr{D}$. The kernel of $G_{\varphi}^{-1}(x)$ on $T_{x} \partial P$ is the linear span of the normals 
$\mu_{r}$ for $r \in \mathscr{F}(x)$. We also denote by $H_{\varphi}(\rho)=\nabla_{\rho}^{2} \varphi\left(e^{\rho}\right)$ the Hessian of the Kähler potential on the open orbit in $\rho$ coordinates. By Legendre duality,

$$
H_{\varphi}(\rho)=G_{\varphi}^{-1}(x), \mu\left(e^{\rho}\right)=x .
$$

This relation may be extended to $\mathscr{D} \rightarrow \partial P$. The kernel of the left side is the Lie algebra of the isotropy group $G_{p}$ of any point $p \in \mu^{-1}(x)$. The volume density has the form

$$
\operatorname{det}\left(G_{\varphi}^{-1}\right)=\delta_{\varphi}(x) \cdot \prod_{r=1}^{d} \ell_{r}(x),
$$
for some positive smooth function $\delta_{\varphi}$ [Abreu 2003]. We note that $\log \prod_{r=1}^{d} \ell_{r}(x)$ is known in convex
optimization as the logarithmic barrier function of $P$.

2C. Kähler potential near $\mathscr{D}$. We also need smooth local Kähler potentials in neighborhoods of points $z_{0} \in \mathscr{D}$. We note that the open orbit Kähler potential (50) is well-defined near $z=0$. Local expressions for the Kähler potential at other points of $\mathscr{D}$ essentially amount to making an affine transformation of $P$ to transform a given corner of $P$ to 0 , and in these coordinates the local Kähler potential near any point of $\mathscr{D}$ can be expressed in the form (50). For instance, on $\mathbb{C} \mathbb{P}^{1}$, a Kähler potential valid at $z=\infty$ is given in the coordinates $w=1 / z$ by $\log \left(1+|w|^{2}\right)$. It differs on the open orbit from the canonical Kähler potential $\log \left(1+|z|^{2}\right)$ by the term $\log |z|^{2}$ whose $i \partial \bar{\partial}$ is a delta function at $z=0$, supported on $\mathscr{D}$ away from the point $w=0$ that one is studying. In [Song 2005] the reader can find further explicit examples of toric Kähler potentials in affine coordinate charts. Hence, in what follows, we will always use (50) as the local expression of the Kähler potential, without explicitly writing in the affine change of variables.

We will however need to be explicit about the use of slice-orbit coordinates $z_{j}^{\prime}, \rho_{j}^{\prime \prime}$ (see (48)) in the local expressions of the Kähler potential. The coordinates near $z_{0}$ depend on $\mathscr{F}_{\epsilon}\left(z_{0}\right)$ from (37). For each $z_{0} \in \mathscr{D}$ corresponding to a codimension $r$ face of $P$, after an affine transformation changing the face to $x^{\prime}=0$, we may write the Kähler potential as the canonical one in slice-orbit coordinates, $F\left(\left|z^{\prime}\right|^{2}, e^{\rho^{\prime \prime}}\right)$ Section 2A (48). Since $0 \in P, F$ is smooth up to the boundary face $z^{\prime}=0$. The fact that $F$ is smooth up to the boundary also follows from the general fact that a smooth $\mathbf{T}^{m}$-invariant function $g \in C_{\mathbf{T}^{m}}^{\infty}(M)$ may be expressed in the form $g(z)=\hat{F}_{g}\left(\mu_{\varphi}(z)\right)$ where as $\hat{F}_{g} \in C^{\infty}\left(\mathbb{R}^{m}\right)$. This is known as the divisibility property of $\mathbf{T}^{m}$-invariant smooth functions [Lerman and Tolman 1997]. It implies that $F$ is a smooth function of the polar coordinates $r_{j}^{2}$ near points of $\mathscr{D}$ in the sense of (49).

2D. Almost analytic extensions. In analyzing the Bergman/Szegó kernel and the functions (21), we make use of the almost analytic extension $\varphi(z, w)$ to $M \times M$ of a Kähler potential for a Kähler $\omega$; for background on almost analytic extensions; see [Boutet de Monvel and Sjöstrand 1976; Melin and Sjöstrand 1975]. It is defined near the totally real antidiagonal $(z, \bar{z}) \in M \times M$ by

$$
\varphi_{\mathbb{C}}(x+h, x+k) \sim \sum_{\alpha, \beta} \frac{\partial^{\alpha+\beta} \varphi}{\partial z^{\alpha} \partial \bar{z}^{\beta}}(x) \frac{h^{\alpha}}{\alpha !} \frac{k^{\beta}}{\beta !} .
$$

When $\varphi$ is real analytic on $M$, the almost analytic extension $\varphi(z, w)$ is holomorphic in $z$ and antiholomorphic in $w$ and is the unique such function for which $\varphi(z)=\varphi(z, z)$. In the general $C^{\infty}$ case, the almost analytic extension is a smooth function with the right side of (59) as its $C^{\infty}$ Taylor expansion 
along the antidiagonal, for which $\bar{\partial} \varphi(z, w)=0$ to infinite order on the antidiagonal. It is only defined in a small neighborhood $(M \times M)_{\delta}=\{(z, w): d(z, w)<\delta\}$ of the antidiagonal in $M \times M$, where $d(z, w)$ refers to the distance between $z$ and $w$ with respect to the Kähler metric $\omega$. It is well defined up to a smooth function vanishing to infinite order on the diagonal; the latter is negligible for our purposes (cf. Proposition 1.1 of [Boutet de Monvel and Sjöstrand 1976].)

The analytic continuation $\varphi(z, w)$ of the Kähler potential was used by Calabi [1953] in the analytic case to define a Kähler distance function, known as the Calabi diastasis function:

$$
D(z, w):=\varphi(z, w)+\varphi(w, z)-(\varphi(z)+\varphi(w)) .
$$

Calabi showed that

$$
D(z, w)=d(z, w)^{2}+O\left(d(z, w)^{4}\right),\left.d d_{w}^{c} D(z, w)\right|_{z=w}=\omega .
$$

One has the same notion in the almost analytic sense.

The gradient of the almost analytic extension of the Kähler potential in the toric case defines the almost analytic extension $\mu_{\mathbb{C}}(z, w)$ of the moment map. We are mainly interested in the case where $w=e^{i \theta} z$ lies on the $\mathbf{T}^{m}$-orbit of $z$, and by (53) we have,

$$
i \mu_{\mathbb{C}}\left(z, e^{i \theta} z\right)=\nabla_{\theta} \varphi_{\mathbb{C}}\left(z, e^{i \theta} z\right)=\nabla_{\theta} F_{\mathbb{C}}\left(e^{i \theta}|z|^{2}\right),
$$

where $F$ is defined in (51). We sometimes drop the subscript in $F_{\mathbb{C}}$ and $\mu_{\mathbb{C}}$ since there is only one interpretation of their extension; but we emphasize that $\varphi\left(z, e^{i \theta} z\right)=F_{\mathbb{C}}\left(e^{i \theta}|z|^{2}\right)$ is very different from $\varphi\left(e^{i \theta} z\right)=F\left(\left|e^{i \theta} z\right|^{2}\right)=F\left(|z|^{2}\right)$. For example, the moment map of the Bargmann-Fock model $\left(\mathbb{C}^{m},|z|^{2}\right)$ is $\mu(z)=\left(\left|z_{1}\right|^{2}, \ldots,\left|z_{m}\right|^{2}\right)$, whose analytic extension is $\left(z_{1} \bar{w}_{1}, \ldots, z_{m} \bar{w}_{m}\right)$. Similarly that of the FubiniStudy metric on $\mathbb{C P}^{m}$ is (in multiindex notation)

$$
\mu_{\mathrm{FS}, \mathbb{C}}(z, w)=\frac{z \cdot \bar{w}}{1+z \cdot \bar{w}} .
$$

In Section $2 \mathrm{~F}$ we further illustrate the notation in the basic examples of Bargmann-Fock and FubiniStudy models. We also observe that (62) continues to hold for the Kähler potential $F\left(\left|z^{\prime}\right|^{2}, e^{\rho^{\prime \prime}}\right)$ in slice-orbit coordinates. That is, we have

$$
i \mu\left(z^{\prime}, e^{\rho^{\prime \prime} / 2}\right)=\left.\nabla_{\theta^{\prime}, \theta^{\prime \prime}} F_{\mathbb{C}}\left(e^{i \theta^{\prime}}\left|z^{\prime}\right|^{2}, e^{i \theta^{\prime \prime}+\rho^{\prime \prime}}\right)\right|_{\left(\theta^{\prime}, \theta^{\prime \prime}\right)=(0,0)} .
$$

The complexified moment map is a map

$$
\mu_{\mathbb{C}} \rightarrow(M \times M)_{\delta} \rightarrow \mathbb{C}^{m} .
$$

The invariance of $\mu$ under the torus action implies that $\mu_{\mathbb{C}}\left(e^{i \theta} z, e^{i \theta} w\right)=\mu_{\mathbb{C}}(z, w)$. The following proposition will clarify the discussion of critical point sets later on (see, for example, Lemma 5.2).

Proposition 2.1. For $\delta$ sufficiently small so that $\mu_{\mathbb{C}}(z, w)$ is well-defined, we have

(1) $\operatorname{Im} \mu_{\mathbb{C}}\left(z, e^{i \theta} z\right)=\frac{1}{2} \nabla_{\theta} D\left(z, e^{i \theta} z\right)$.

(2) $\mu_{\mathbb{C}}\left(z, e^{i \theta} z\right)=\mu_{\mathbb{C}}(z, z)$ with $\left(z, e^{i \theta} z\right) \in(M \times M)_{\delta}$ if and only if $e^{i \theta} z=z$. 
Proof. The proof of the identity (1) is immediate from the definitions; we only note that the diastasis function is a kind of real part, and that the imaginary part originates in the factor of $i$ in (62). One can check the factors of $i$ in the Bargmann-Fock model, where $\mu_{\mathbb{C}}\left(z, e^{i \theta} z\right)=e^{i \theta}|z|^{2}$ while $D\left(z, e^{i \theta} z\right)=$ $2(\cos \theta-1)|z|^{2}+2 i(\sin \theta)|z|^{2}$ (in vector notation).

By (61), $D(z, w)$ has a strict global minimum at $w=z$ which is nondegenerate. It is therefore isolated for each $z$. Since its Hessian at $w=z$ is the identify with respect to $\omega$, the isolating neighborhood has a uniform size as $z$ varies. Thus, there exists a $\delta>0$ so that $\mu_{\mathbb{C}}(z, w)=\mu_{\mathbb{C}}(z, z)$ in $(M \times M)_{\delta}$ if and only if $z=w$. This is true both in the real analytic case and the almost-analytic case.

2E. Hilbert spaces of holomorphic sections. On the "quantum level", a toric Kähler variety $(M, \omega)$ induces the sequence of spaces $H^{0}\left(M, L^{k}\right)$ of holomorphic sections of powers of the holomorphic toric line bundle $L$ with $c_{1}(L)=\frac{1}{2 \pi}[\omega]$. The $\left(\mathbb{C}^{*}\right)^{m}$ action lifts to $H^{0}\left(M, L^{k}\right)$ as a holomorphic representation which is unitary on $\mathbf{T}^{m}$. Corresponding to the lattice points $\alpha \in k P$, there is a natural basis $\left\{s_{\alpha}\right\}$ (denoted $\chi_{\alpha}^{P}$ in [Shiffman et al. 2004]) of $H^{0}\left(M, L^{k}\right)$ given by joint eigenfunctions of the $\left(\mathbb{C}^{*}\right)^{m}$ action. It is well-known that the joint eigenvalues are precisely the lattice points $\mathbb{Z}^{m} \cap k P$ in the $k$-th dilate of $P$. On the open orbit $s_{\alpha}(z)=\chi_{\alpha}(z) e^{k}$ where $e$ is a frame and where as above $\chi_{\alpha}(z)=z^{\alpha}=z_{1}^{\alpha_{1}} \cdots z_{m}^{\alpha_{m}}$. Hence, the $s_{\alpha}$ are referred to as monomials. For further background, we refer to [Shiffman et al. 2004]. A hermitian metric $h$ on $L$ induces the Hilbert space inner products (6) on $H^{0}\left(M, L^{k}\right)$.

As is evident from (21), we will need formulae for the monomials which are valid near $\mathscr{D}$. By (40) and (42), we have

$$
\chi_{\alpha^{j}}(z)=\eta_{j}(z) \chi_{v^{0}}(z), \quad z \in\left(\mathbb{C}^{*}\right)^{m}
$$

and by (43) we then have

$$
\left|\chi_{\alpha}(z)\right|^{2}=\left|\eta^{\tilde{\Gamma}(\alpha)}\right|^{2}
$$

As mentioned above, for simplicity of notation we suppress the transformation $\tilde{\Gamma}$ and coordinates $\eta$, and we will use the orbit-slice coordinates of (48). Thus, we denote the monomials corresponding to lattice points $\alpha$ near a face $F$ by $\left(z^{\prime}\right)^{\alpha^{\prime}} e^{\left\langle\left(i \theta^{\prime \prime}+\rho^{\prime \prime} / 2\right), \alpha^{\prime \prime}\right\rangle}$, where $\tilde{\Gamma}(\alpha)=\left(\alpha^{\prime}, \alpha^{\prime \prime}\right)$ with $\alpha^{\prime \prime}$ in the coordinate hyperplane corresponding under $\tilde{\Gamma}$ to $F$ and with $\alpha^{\prime}$ in the normal space.

2F. Examples: Bargmann-Fock and Fubini-Study models. As mentioned above the Bargmann-Fock model is the linear model. It plays a fundamental role in this article because it provides an approximation for objects on any toric variety on balls of radius $\log k / \sqrt{k}$ and also near $\mathscr{D}$. Although it and the FubiniStudy model are elementary examples, we go over them because the notation is used frequently later on.

The Bargmann-Fock models on $\mathbb{C}^{m}$ correspond to choices of a positive definite hermitian matrix $H$ on $\mathbb{C}^{m}$. A toric Bargmann-Fock model is one in which $H$ commutes with the standard $\mathbf{T}^{m}$ action, that is, is a diagonal matrix. We denote its diagonal elements by $H_{j \bar{j}}$. The Kähler metric on $\mathbb{C}^{m}$ is thus $i \partial \bar{\partial} \varphi_{B F, H}(z)$ where the global Kähler potential is

$$
\varphi_{B F, H}(z)=\sum_{j=1}^{m} H_{j j}\left|z_{j}\right|^{2}=F\left(\left|z_{1}\right|^{2}, \ldots,\left|z_{m}\right|^{2}\right), \text { with } F\left(y_{1}, \ldots, y_{m}\right)=\sum_{j} H_{j \bar{j}} y_{j} .
$$

For simplicity we often only consider the case $H=I$. Putting $\left|z_{j}\right|^{2}=e^{\rho_{j}}$ and using (53), it follows that $\mu_{B F, H}\left(z_{1}, \ldots, z_{m}\right)=\left(H_{1 \overline{1}}\left|z_{1}\right|^{2}, \ldots, H_{m \bar{m}}\left|z_{m}\right|^{2}\right): \mathbb{C}^{m} \rightarrow \mathbb{R}_{+}^{m}$ as in (38). The symplectic potential 
Legendre dual to $\varphi_{B F, H}$ is given by

$$
u_{B F, H}(x)=-\varphi_{B F, H}\left(\mu_{B F}^{-1}(x)\right)+2\left\langle\log \mu_{B F, H}^{-1}(x), x\right\rangle=-\sum_{j} x_{j}+\sum_{j=1}^{m} x_{j} \log \frac{x_{j}}{H_{j \bar{j}}} .
$$

In this case, $G_{B F, H}$ is the diagonal matrix with entries $\frac{1}{x_{j} H_{j \bar{j}}}$, so

$$
\operatorname{det} G_{B F, H}=\frac{1}{\operatorname{det} H} \prod_{j} \frac{1}{x_{j}} .
$$

The off-diagonal analytic extension of the Kähler potential in the sense of (59) is then

$$
\varphi_{B F, H}(z, \bar{w})=\sum_{j=1}^{m} H_{j j} z_{j} \bar{w}_{j}=F\left(z_{1} \bar{w}_{1}, \ldots, z_{m} \bar{w}_{m}\right),
$$

and in particular,

$$
\varphi_{B F, H}\left(z, e^{i \theta} z\right)=\sum_{j=1}^{m} H_{j j} e^{i \theta_{j}}\left|z_{j}\right|^{2}=F\left(e^{i \theta_{1}}\left|z_{1}\right|^{2}, \ldots, e^{i \theta_{m}}\left|z_{m}\right|^{2}\right) .
$$

Henceforth we often write the right side in the multiindex notation $F_{\mathbb{C}}\left(e^{i \theta}|z|^{2}\right)$. We observe, as claimed in (62), that $\left.\nabla_{\theta} F_{B F, \mathbb{C}}\left(e^{i \theta}|z|^{2}\right)\right|_{\theta=0}=i \mu_{B F}(z)$.

Quantization of the Bargmann-Fock model with $H=I$ produces the Bargmann-Fock (Hilbert) space

$$
\mathscr{H}^{2}\left(\mathbb{C}^{m},(2 \pi)^{-m} k^{m} e^{-k|z|^{2}} d z \wedge d \bar{z}\right)
$$

of entire functions which are $L^{2}$ relative to the displayed weight. It is infinite-dimensional and a basis is given by the monomials $z^{\alpha}$ where $\alpha \in \mathbb{R}_{+}^{m} \cap \mathbb{Z}^{m}$. In Section $3 \mathrm{~A}$ we compute their $L^{2}$ norms. For $H \neq I$ one uses the volume form $e^{-k\langle H z, z\rangle}(i \partial \bar{\partial}\langle H z, z\rangle)^{m} / m !=e^{-k\langle H z, z\rangle} \operatorname{det} H d z \wedge d \bar{z}$.

Toric Fubini-Study metrics provide compact models which are similar to Bargmann-Fock models. In a local analysis we always use the latter. A Fubini-Study metric on $\mathbb{C P}^{m}$ is determined by a positive hermitian form $H$ on $\mathbb{C}^{m+1}$ and a toric Fubini-Study metric is a diagonal one $\sum_{j=0}^{m} H_{j j}\left|Z_{j}\right|^{2}$. In the affine chart $Z_{0} \neq 0$, for example, a local Fubini-Study Kähler potential is

$$
\varphi_{\mathrm{FS}, H}\left(z_{1}, \ldots, z_{m}\right)=\log \left(1+\sum_{j} h_{j \bar{j}}\left|z_{j}\right|^{2}\right),
$$

where $h_{j \bar{j}}=H_{j \bar{j}} / H_{0 \overline{0}}$. This is a valid Kähler potential near $z=0$ but of course has logarithmic singularities on the hyperplane at infinity. The almost analytic extension of the Fubini-Study Kähler potential is given in the affine chart by $\log \left(1+\sum_{j} h_{j j} z_{j} \bar{w}_{j}\right)$. Thus (62) asserts that

$$
i \frac{\sum_{j} h_{j \bar{j}}\left|z_{j}\right|^{2}}{1+\sum_{j} h_{j \bar{j}}\left|z_{j}\right|^{2}}=\left.\nabla_{\theta} \log \left(1+\sum_{j} h_{j \bar{j}} e^{i \theta_{j}}\left|z_{j}\right|^{2}\right)\right|_{\theta=0} .
$$

Quantization produces the Hilbert spaces $H^{0}\left(\mathbb{C P} \mathbb{P}^{m}, \mathscr{O}(k)\right)$, where $\mathbb{O}(k) \rightarrow \mathbb{C} \mathbb{P}^{m}$ is the $k t h$ power of the hyperplane section bundle. Sections lift to homogeneous holomorphic polynomials on $\mathbb{C}^{m+1}$, and correspond to lattice points in $k \Sigma$ where $\Sigma$ is the unit simplex in $\mathbb{R}^{m}$. 
2G. Linearization of the Monge-Ampère equation. It is known that the Legendre transform linearizes the Monge-Ampère geodesic equation. Since it is important for this article, we present a simple proof that does not seem to exist in the literature.

Proposition 2.2. Let $M_{P}^{c}$ be a toric variety. Then under the Legendre transform $\varphi \mapsto u_{\varphi}$ the complex Monge-Ampère equation on $\mathcal{H}_{\mathbf{T}^{m}}$ linearizes to the equation $u^{\prime \prime}=0$. Hence the Legendre transform of a geodesic $\varphi_{t}$ has the form $u_{t}=u_{0}+t\left(u_{1}-u_{0}\right)$.

Proof. It suffices to show that the energy functional

$$
E=\int_{0}^{1} \int_{M} \dot{\varphi}_{t}^{2} d \mu_{\varphi_{t}} d t
$$

is Euclidean on paths of symplectic potentials. For each $t$ let us push forward the integral $\int_{M} \dot{\varphi}_{t}^{2} d \mu_{\varphi}$ under the moment map $\mu_{\varphi_{t}}$. The integrand is by assumption invariant under the real torus action, so the push forward is a diffeomorphism on the real points. The volume measure $d \mu_{\varphi_{t}}$ pushes forward to $d x$. The function $\partial_{t} \varphi_{t}(\rho)$ pushes forward to the function $\psi_{t}(x)=\dot{\varphi}_{t}\left(\rho_{x, t}\right)$ where $\mu_{\varphi_{t}}\left(\rho_{x, t}\right)=x$. By (55), the symplectic potential at time $t$ is

$$
u_{t}(x)=\left\langle x, \rho_{x, t}\right\rangle-\varphi_{t}\left(\rho_{x, t}\right)
$$

We note that

$$
\dot{u}_{t}=\left\langle x, \partial_{t} \rho_{x, t}\right\rangle-\dot{\varphi}_{t}\left(\rho_{x, t}\right)-\left\langle\nabla_{\rho} \varphi_{t}\left(\rho_{x, t}\right), \partial_{t} \rho_{x, t}\right\rangle .
$$

The outer terms cancel, and thus, our integral is just

$$
\int_{0}^{1} \int_{P}\left|\dot{u}_{t}\right|^{2} d x d t
$$

Clearly the Euler-Lagrange equations are linear.

\section{The functions $\mathscr{P}_{h^{k}}$ and $\mathscr{Q}_{h^{k}}$}

We now introduce the key players in the analysis, the norming constants $2_{h^{k}}(\alpha)(20)$ and the dual constants $\mathscr{P}_{h^{k}}(\alpha)$ of (22). The duality is given in the following:

Proposition 3.1.

$$
2_{h_{k}}(\alpha)=\frac{e^{k u_{\varphi}(\alpha / k)}}{\mathscr{P}_{h^{k}}(\alpha)} .
$$

Proof. By (55), it follows that

$$
\left\|s_{\alpha}\left(\mu_{h}^{-1}\left(\frac{\alpha}{k}\right)\right)\right\|_{h^{k}}^{2}=\left|\chi_{\alpha}\left(\mu_{h}^{-1}\left(\frac{\alpha}{k}\right)\right)\right|^{2} e^{-k \varphi_{h}\left(\mu_{h}^{-1}(\alpha / k)\right)}=e^{k u_{\varphi_{h}}(\alpha / k)} .
$$

Corollary 3.2.

$$
\mathscr{R}_{k}(t, \alpha)=\frac{\left(\mathscr{P}_{h_{0}^{k}}(\alpha)\right)^{1-t}\left(\mathscr{P}_{h_{1}^{k}}(\alpha)\right)^{t}}{\mathscr{P}_{h_{t}^{k}}(\alpha)}
$$

Proof. We need to show that

$$
\frac{\mathscr{2}_{h_{t}^{k}}(\alpha)}{\left(\mathscr{2}_{h_{0}^{k}}(\alpha)\right)^{1-t}\left(\mathscr{2}_{h_{1}^{k}}(\alpha)\right)^{t}}=\frac{\left(\mathscr{P}_{h_{0}^{k}}(\alpha)\right)^{1-t}\left(\mathscr{P}_{h_{1}^{k}}(\alpha)\right)^{t}}{\mathscr{P}_{h_{t}^{k}}(\alpha)}
$$


By Proposition 3.1, the left side of (71) equals

$$
\frac{\left|\chi_{\alpha}\left(\mu_{h_{t}}^{-1}\left(\frac{\alpha}{k}\right)\right)\right|^{2} e^{-k \varphi_{t}\left(\mu_{h_{t}}^{-1}(\alpha / k)\right)}}{\mathscr{P}_{h_{t}^{k}}(\alpha)}\left(\frac{\mathscr{P}_{h_{0}^{k}}(\alpha)}{\left|\chi_{\alpha}\left(\mu_{0}^{-1}\left(\frac{\alpha}{k}\right)\right)\right|^{2} e^{-k \varphi_{0}\left(\mu_{0}^{-1}(\alpha / k)\right)}}\right)^{1-t}\left(\frac{\mathscr{P}_{h_{1}^{k}}(\alpha)}{\left|\chi_{\alpha}\left(\mu_{1}^{-1}\left(\frac{\alpha}{k}\right)\right)\right|^{2} e^{-k \varphi_{1}\left(\mu_{1}^{-1}(\alpha / k)\right)}}\right)^{t} .
$$

By the equality in the proof of Proposition 3.1, the left side of (71) equals

$$
e^{k\left(u_{t}(\alpha / k)+(1-t) u_{0}(\alpha / k)+t u_{1}(\alpha / k)\right)} \times \frac{\left(\mathscr{P}_{h_{0}^{k}}(\alpha)\right)^{1-t}\left(\mathscr{P}_{h_{1}^{k}}(\alpha)\right)^{t}}{\mathscr{P}_{h_{t}^{k}}(\alpha)} .
$$

But $u_{t}(x)+(1-t) u_{0}(x)+t u_{1}(x)=0$ on a toric variety, and this gives the stated equality.

Further, we relate the full $\mathscr{P}_{h^{k}}(\alpha, z)$ to the Szegő kernel. The Szegő (or Bergman) kernels of a positive hermitian line bundle $(L, h) \rightarrow(M, \omega)$ over a Kähler manifold are the kernels of the orthogonal projections $\Pi_{h^{k}}: L^{2}\left(M, L^{k}\right) \rightarrow H^{0}\left(M, L^{k}\right)$ onto the spaces of holomorphic sections with respect to the inner product $\operatorname{Hilb}_{k}(h)(6)$. Thus, we have

$$
\Pi_{h^{k}} s(z)=\int_{M} \Pi_{h^{k}}(z, w) \cdot s(w) \frac{\omega_{h}^{m}}{m !},
$$

where the denotes the $h$-hermitian inner product at $w$. Let $e_{L}$ be a local holomorphic frame for $L \rightarrow M$ over an open set $U \subset M$ of full measure, and let $\left\{s_{j}^{k}=f_{j} e_{L}^{\otimes k}: j=1, \ldots, d_{k}\right\}$ be an orthonormal basis for $H^{0}\left(M, L^{k}\right)$ with $d_{k}=\operatorname{dim} H^{0}\left(M, L^{k}\right)$. Then the Szegó kernel can be written in the form

$$
\Pi_{h^{k}}(z, w):=F_{h^{k}}(z, w) e_{L}^{\otimes k}(z) \otimes \overline{e_{L}^{\otimes k}(w)},
$$

where

$$
F_{h^{k}}(z, w)=\sum_{j=1}^{d_{k}} f_{j}(z) \overline{f_{j}(w)}
$$

Since the Szegó kernel is a section of the bundle $\left(L^{k}\right) \otimes\left(L^{k}\right)^{*} \rightarrow M \times M$, it often simplifies the analysis to lift it to a scalar kernel $\hat{\Pi}_{h^{k}}(x, y)$ on the associated unit circle bundle $X \rightarrow M$ of $(L, h)$. Here, $X=\partial D_{h}^{*}$ is the boundary of the unit disc bundle with respect to $h^{-1}$ in the dual line bundle $L^{*}$. We use local product coordinates $x=(z, t) \in M \times S^{1}$ on $X$, where $x=e^{i t}\left\|e_{L}(z)\right\|_{h} e_{L}^{*}(z) \in X$. To avoid confusing the $S^{1}$ action on $X$ with the $\mathbf{T}^{m}$ action on $M$ we use $e^{i t}$ for the former and $e^{i \theta}$ (multiindex notation) for the latter. We note that the $\mathbf{T}^{m}$ action lifts to $X$ and combines with the $S^{1}$ action to produce a $\left(S^{1}\right)^{m+1}$ action. We refer to [Zelditch 1998; 2009; Shiffman and Zelditch 2002] for background and for more on lifting the Szegő kernel of a toric variety.

The equivariant lift of a section $s=f e_{L}^{\otimes k} \in H^{0}\left(M, L^{k}\right)$ is given explicitly by

$$
\hat{s}(z, t)=e^{i k t}\left\|e_{L}^{\otimes k}\right\|_{h^{k}} f(z)=e^{k[-(1 / 2) \varphi(z)+i t]} f(z) .
$$

The Szegó kernel thus lifts to $X \times X$ as the scalar kernel

$$
\hat{\Pi}_{k}\left(z, t ; w, t^{\prime}\right)=e^{k\left[-(1 / 2) \varphi(z)-(1 / 2) \varphi(w)+i\left(t-t^{\prime}\right)\right]} F_{k}(z, w) .
$$

Since it is $S^{1}$ - equivariant we often put $t=t^{\prime}=0$. 
Proposition 3.3.

$$
\mathscr{P}_{h^{k}}(\alpha, z)=(2 \pi)^{-m} \int_{\mathbf{T}^{m}} \hat{\Pi}_{h^{k}}\left(e^{i \theta} z, 0 ; z, 0\right) e^{-i\langle\alpha, \theta\rangle} d \theta .
$$

Proof. We recall that $\chi_{\alpha}(z)=z^{\alpha}$ is the local representative of $s_{\alpha}$ in the open orbit with respect to an invariant frame. Since $\left\{\chi_{\alpha} / \sqrt{2_{h^{k}}(\alpha)}\right\}$ is the local expression of an orthonormal basis, we have

$$
F_{h^{k}}(z, w)=\sum_{\alpha \in k P \cap \mathbb{Z}^{m}} \frac{\chi_{\alpha}(z) \overline{\chi_{\alpha}(w)}}{2_{h^{k}}(\alpha)}
$$

hence

$$
\hat{\Pi}_{h^{k}}(z, 0 ; w, 0)=\sum_{\alpha \in k P \cap \mathbb{Z}^{m}} \frac{\chi_{\alpha}(z) \overline{\chi_{\alpha}(w)} e^{-k(\varphi(z)+\varphi(w)) / 2}}{2_{h^{k}}(\alpha)} .
$$

It follows that

$$
\Pi_{h^{k}}\left(e^{i \theta} z, 0 ; z, 0\right)=\sum_{\alpha \in k P \cap \mathbb{Z}^{m}} \frac{\left|\chi_{\alpha}(z)\right|^{2} e^{-k \varphi(z)} e^{i\langle\alpha, \theta\rangle}}{2_{h^{k}}(\alpha)} .
$$

Integrating against $e^{-i\langle\alpha, \theta\rangle}$ sifts out the $\alpha$ term.

Corollary 3.4. We have

$$
\mathscr{P}_{h^{k}}(\alpha)=(2 \pi)^{-m} \int_{\mathbf{T}^{m}} \hat{\Pi}_{h^{k}}\left(e^{i \theta} \mu_{h}^{-1}\left(\frac{\alpha}{k}\right), 0 ; \mu_{h}^{-1}\left(\frac{\alpha}{k}\right), 0\right) e^{-i\langle\alpha, \theta\rangle} d \theta
$$

3A. Bargmann-Fock model. As discussed in Section $2 \mathrm{~F}$, the Hilbert space in this model has the orthogonal basis $z^{\alpha}$ with $\alpha \in \mathbb{R}_{+}^{m} \cap \mathbb{Z}^{m}$. The Bargmann-Fock norming constants when $H=I$ are given by

$$
2_{h_{B F}^{k}}(\alpha)=k^{-|\alpha|-m} \alpha !, \quad \text { where } \alpha !:=\alpha_{1} ! \ldots \alpha_{m} ! .
$$

It follows that an orthonormal basis of holomorphic monomials is given by $\left\{k^{(|\alpha|+m / 2)} z^{\alpha} / \sqrt{\alpha !}\right\}$.

We therefore have

$$
\frac{\left|s_{\alpha}(z)\right|_{h_{B F}^{k}}^{2}}{2_{h_{B F}^{k}}(\alpha)}=k^{|\alpha|+m} \frac{\left|z^{\alpha}\right|^{2}}{\alpha !} e^{-k|z|^{2}},
$$

and in particular,

$$
\mathscr{P}_{h_{B F}^{k}}(\alpha)=k^{m} e^{-|\alpha|} \frac{\alpha^{\alpha}}{\alpha !},
$$

where $\alpha^{\alpha}=1$ when $\alpha=0$. Here, we use that $u_{B F}\left(\frac{\alpha}{k}\right)=\frac{\alpha}{k} \log \frac{\alpha}{k}-\frac{\alpha}{k}$, so that

$$
e^{k u_{B F}(\alpha / k)}=e^{-|\alpha|} \frac{k^{-|\alpha|}}{\alpha^{\alpha}}
$$

and $\mathscr{2}_{h_{B F}^{k}}(\alpha)=k^{-m-|\alpha|} \alpha$ !. We observe that $\mathscr{P}_{h_{B F}^{k}}(\alpha)$ depends on $k$ only through the factor $k^{m}$.

Precisely the same formula holds if we replace $I$ by a positive diagonal $H$ with elements $H_{j \bar{j}}$. By a change of variables we obtain $2_{h_{B F, H}^{k}}(\alpha)=\prod_{j=1}^{m} H_{j \bar{j}}^{-\alpha_{j}} 2_{h_{B F}^{k}}(\alpha)$, and also by (67) we have $u_{B F, H}(x)=$ $u_{B F}(x)+\sum_{j} x_{j} \log H_{j j}$. Hence, by Proposition 3.1,

$$
\mathscr{P}_{h_{B F, H}^{k}}(\alpha)=\mathscr{P}_{h_{B F}^{k}}(\alpha) \prod_{j=1}^{m} H_{j \bar{j}}^{-\alpha_{j}} e^{\sum_{j} \alpha_{j} \log H_{j \bar{j}}}=\mathscr{P}_{h_{B F}^{k}}(\alpha) .
$$


3A1. $\mathbb{C P}^{m}$. In the Fubini-Study model, a basis of $H^{0}\left(\mathbb{C} \mathbb{P}^{m}, \mathscr{O}(k)\right)$ is given by monomials with $\alpha \in k \Sigma$ (see Section $2 \mathrm{~F}$ ), and the norming constants are given by

$$
2_{h_{\mathrm{FS}}^{k}}(\alpha)=\left(\begin{array}{l}
k \\
\alpha
\end{array}\right):=\left(\begin{array}{c}
k \\
\alpha_{1}, \ldots, \alpha_{m}
\end{array}\right)^{-1} .
$$

Recall that multinomial coefficients are defined for $\alpha_{1}+\cdots+\alpha_{m} \leq k$ by

$$
\left(\begin{array}{c}
k \\
\alpha_{1}, \ldots, \alpha_{m}
\end{array}\right)=\frac{k !}{\alpha_{1} ! \cdots \alpha_{m} !(k-|\alpha|) !},
$$

where, as above, $|\alpha|=\alpha_{1}+\cdots+\alpha_{m}$.

We further have

$$
\left|s_{\alpha}(z)\right|_{h_{\mathrm{FS}}^{k}}^{2}=\left|z^{\alpha}\right|^{2} e^{-k \log \left(1+|z|^{2}\right)}
$$

and therefore,

$$
\mathscr{P}_{h_{\mathrm{FS}}^{k}}(\alpha, z)=\left(\begin{array}{c}
k \\
\alpha_{1}, \ldots, \alpha_{m}
\end{array}\right)\left|z^{\alpha}\right|^{2} e^{-k \log \left(1+|z|^{2}\right)}
$$

and since

$$
e^{-k u_{\mathrm{FS}}(\alpha / k)}=\left|s_{\alpha}\left(\mu_{\mathrm{FS}}^{-1}\left(\frac{\alpha}{k}\right)\right)\right|_{h_{\mathrm{FS}}^{k}}^{2}=\left(\frac{\alpha}{k}\right)^{\alpha}\left(1-\frac{|\alpha|}{k}\right)^{k-|\alpha|},
$$

we have

$$
\mathscr{P}_{h_{\mathrm{FS}}^{k}}(\alpha)=\frac{k !}{\alpha_{1} ! \cdots \alpha_{m} !(k-|\alpha|) !}\left(\frac{\alpha}{k}\right)^{\alpha}\left(1-\frac{|\alpha|}{k}\right)^{k-|\alpha|}
$$

\section{The Szegó kernel of a toric variety}

We will use Proposition 3.3 to reduce the joint asymptotics of $\left.\mathscr{P}_{h^{k}} \alpha, z\right)$ in $(k, \alpha)$ to asymptotics of the Bergman-Szegó kernel off the diagonal. We now review some general facts about diagonal and offdiagonal expansions of these kernels, for which complete details can be found in [Shiffman and Zelditch 2002], and we also consider some special properties of toric Bergman-Szegó kernels which are very convenient for calculations; to some extent they derive from [Shiffman et al. 2004], but the latter only considered Szegó kernels for powers of Bergman metrics.

The Szegó kernels $\hat{\Pi}_{h^{k}}(x, y)$ are the Fourier coefficients of the total Szegó projector $\hat{\Pi}_{h}(x, y)$ : $\mathscr{L}^{2}(X) \rightarrow \mathscr{H}^{2}(X)$, where $\mathscr{H}^{2}(X)$ is the Hardy space of boundary values of holomorphic functions on $D^{*}$ (the kernel of $\bar{\partial}_{b}$ in $L^{2}(X)$ ). Thus,

$$
\hat{\Pi}_{h^{k}}(x, y)=\frac{1}{2 \pi} \int_{0}^{2 \pi} e^{-i k t} \hat{\Pi}_{h}\left(e^{i t} x, y\right) d t .
$$

The properties we need of $\hat{\Pi}_{h^{k}}(x, y)$ are based on the Boutet de Monvel-Sjöstrand construction [1976] of an oscillatory integral parametrix for the Szegó kernel:

$$
\hat{\Pi}(x, y)=S(x, y)+E(x, y),
$$

with $S(x, y)=\int_{0}^{\infty} e^{i \lambda \psi(x, y)} s(x, y, \lambda) d \lambda, \quad E(x, y) \in \mathscr{C}^{\infty}(X \times X)$. 
The phase function $\psi$ is of positive type and is given in the local coordinates above by

$$
\psi\left(z, t ; w, t^{\prime}\right)=\frac{1}{i}\left(1-e^{\varphi(z, w)-(\varphi(z)+\varphi(w)) / 2} e^{i\left(t-t^{\prime}\right)}\right) .
$$

Here, $\varphi(z, w)$ is the almost analytic extension of the local Kähler potential with respect to the frame, that is, $h=e^{-\varphi(z)}$; see (59) for the notion of almost analytic extension. The amplitude $s\left(z, t ; w, t^{\prime}, \lambda\right)$ is a semiclassical amplitude as in [Boutet de Monvel and Sjöstrand 1976, Theorem 1.5], that is, it admits an expansion $s \sim \sum_{j=0}^{\infty} \lambda^{m-j} s_{j}(x, y) \in S^{m}\left(X \times X \times \mathbb{R}^{+}\right)$.

The phase $\psi\left(z, t ; w, t^{\prime}\right)$ is the generating function for the graph of the identity map along the symplectic cone $\Sigma \subset T^{*} X$ defined by $\Sigma=\left\{\left(x, r \alpha_{x}\right): r>0\right\}$, where $\alpha_{x}$ is the Chern connection one form. Hence the singularity of $\hat{\Pi}(x, y)$ only occurs on the diagonal and the symbol $s$ is understood to be supported in a small neighborhood $(M \times M)_{\delta}$ of the antidiagonal. It will be useful to make the cutoff explicit by introducing a smooth cutoff function $\chi(d(z, w))$, where $\chi$ is a smooth even function on $\mathbb{R}$ and $d(z, w)$ denotes the distance between $z, w$ in the base Kähler metric.

As above, we denote the $k$-th Fourier coefficient of these operators relative to the $S^{1}$ action by $\hat{\Pi}_{h^{k}}=$ $S_{h^{k}}+E_{h^{k}}$. Since $E$ is smooth, we have $E_{h^{k}}(x, y)=O\left(k^{-\infty}\right)$, where $O\left(k^{-\infty}\right)$ denotes a quantity which is uniformly $O\left(k^{-n}\right)$ on $X \times X$ for all positive $n$. Hence $E_{h^{k}}(z, w)$ is negligible for all the calculations and estimates of this article, and further it is only necessary to use a finite number of terms of the symbol $s$. For simplicity of notation, we will use the entire symbol.

It follows that (with $x=(z, t), y=(w, 0)$ and with $\chi(d(z, w))$ as above ),

$$
\begin{aligned}
\hat{\Pi}_{h^{k}}(x, y) & =S_{h^{k}}(x, y)+O\left(k^{-\infty}\right) \\
& =k \int_{0}^{\infty} \int_{0}^{2 \pi} e^{i k(-t+\lambda \psi(z, t ; w, 0))} \chi(d(z, w)) s(z, t ; w, 0, k \lambda) d t d \lambda+O\left(k^{-\infty}\right) .
\end{aligned}
$$

The integral is a damped complex oscillatory integral since (61) implies that

$$
\operatorname{Im} \psi(x, y) \geq C d(x, y)^{2}, \quad(x, y \in X),
$$

for $(x, y)$ sufficiently close to the diagonal, as one sees by Taylor expanding the phase around the diagonal; see [Boutet de Monvel and Sjöstrand 1976, Corollary 1.3]. It follows from (83) and from (84) that the Szegô kernel $\Pi_{h^{k}}(z, w)$ on $M$ is "gaussian" in small balls $d(z, w) \leq \log k / \sqrt{k}$, that is,

$$
\left|\hat{\Pi}_{h^{k}}\left(z, \varphi ; w, \varphi^{\prime}\right)\right| \leq C k^{m} e^{-k d(z, w)^{2}}+O\left(k^{-\infty}\right), \quad \text { when } d(z, w) \leq \frac{\log k}{\sqrt{k}}
$$

and on the complement $d(z, w) \geq \log k / \sqrt{k}$ it is rapidly decaying. This rapid decay can be improved to long range (subgaussian) exponential decay off the diagonal given by the global Agmon estimates,

$$
\left|\hat{\Pi}_{h^{k}}\left(z, \varphi ; w, \varphi^{\prime}\right)\right| \leq C k^{m} e^{-\sqrt{k} d(z, w)} .
$$

We refer to [Christ 2003; Lindholm 2001] for background and references.

It is helpful to eliminate the integrals in (83) by complex stationary phase. Expressed in a local frame and local coordinates on $M$, the result is this:

Proposition 4.1. Let $(L, h)$ be a $C^{\infty}$ positive hermitian line bundle, and let $h=e^{-\varphi}$ in a local frame. Then in this frame, there exists a semiclassical amplitude $A_{k}(z, w) \sim k^{m} a_{0}(z, w)+k^{m-1} a_{1}(z, w)+\cdots$ 
in the parameter $k^{-1}$ such that

$$
\hat{\Pi}_{h^{k}}(z, 0 ; w, 0)=e^{k(\varphi(z, w)-(\varphi(z)+\varphi(w)) / 2)} \chi_{k}(d(z, w)) A_{k}(z, w)+O\left(k^{-\infty}\right),
$$

where, as above, $\chi_{k}(d(z, w))=\chi\left(\frac{k^{1 / 2}}{\log k} d(z, w)\right)$ is a cutoff to a $\frac{\log k}{\sqrt{k}}$-neighborhood of the diagonal.

Proof. This follows from the scaling asymptotics of [Shiffman and Zelditch 2002] or from [Berman et al. 2008, Theorem 3.1]. We refer there for a detailed proof of the scaling asymptotics and only sketch a somewhat intuitive proof.

The integral (83) is a complex oscillatory integral with a positive complex phase. With no loss of generality we may set $\varphi^{\prime}=0$. Taking the $\lambda$-derivative gives one critical point equation

$$
1-e^{\varphi(z, w)-(\varphi(z)+\varphi(w)) / 2} e^{i t}=0
$$

and the critical point equation in $t$ implies that $\lambda=1$. The $\lambda$-critical point equation can only be satisfied for complex $t$ with imaginary part equal to the negative of the Calabi diastasis function (60), that is,

$$
\operatorname{Im} t=D(z, w)
$$

and with real part equal to $-\operatorname{Im} \varphi(z, w)$. To obtain asymptotics, we therefore have to deform the integral over $S^{1}$ to the circle $|\zeta|=e^{-D(z, w)}$. Since $d(z, w) \leq C(\log k / \sqrt{k})$ by assumption, the deformed contour is a slightly rescaled circle by the amount $(\log k / \sqrt{k})$; in the complete proofs, the contour is held fixed and the integrand is rescaled as in [Shiffman and Zelditch 2002]. The contour deformation is possible modulo an error $O\left(k^{-M}\right)$ of arbitrarily rapid polynomial decay because the integrand may be replaced by the parametrix (up to any order in $\lambda$ ) which has a holomorphic dependence on the $\mathbb{C}^{*}$ action on $L^{*}$, hence in $e^{i \theta}$ to a neighborhood of $S^{1}$ in $\mathbb{C}$. This is immediately visible in the phase and with more work is visible in the amplitude (this is the only incompleteness in the proof; the statement can be derived from [Shiffman and Zelditch 2002; Christ 2003]). We need to use a cutoff to a neighborhood of the diagonal of $M \times M$, but it may be chosen to be independent of $\theta$.

By deforming the circle of integration from the unit circle to $|\zeta|=e^{D(z, w)}$ and then changing variables $t \mapsto t+i D(z, w)$ to bring it back to the unit circle, we obtain

$$
\hat{\Pi}_{h^{k}}(x, y) \sim k \int_{0}^{\infty} \int_{0}^{2 \pi} e^{i k(-t-i D(z, w)-\lambda \psi(z, t+i D(z, w) ; w, 0))} s(z, t+i D(z, w) ; w, 0, k \lambda) d t d \lambda \bmod k^{-\infty} .
$$

The new critical point equations state that $\lambda=1$ and that $e^{i \operatorname{Im} \varphi(z, w)} e^{i t}=1$. The calculation shows that $\psi=0$ on the critical set so the phase factor on the critical set equals $e^{\varphi(z, w)-(1 / 2)(\varphi(z)+\varphi(w))}$. The Hessian of the phase on the critical set is $\left(\begin{array}{ll}0 & 1 \\ 1 & i\end{array}\right)$, as in the diagonal case, and the rest of the calculation proceeds as in [Zelditch 1998]. (As mentioned above, a complete proof is contained in [Shiffman and Zelditch 2002]).

4A. Toric Bergman-Szegó kernels. In the toric case, we may simplify the expression for the Szegő kernels in Proposition 4.1 using the almost analytic extension (see Equation (59)) of the Kähler potential $\varphi(z, w)$ to $M \times M$, which has the form

$$
F_{\mathbb{C}}(z \cdot \bar{w})=\text { the almost analytic extension of } F\left(|z|^{2}\right) \text { to } M \times M .
$$


The almost analytic extension will be illustrated in some analytic examples below, where it coincides with the analytic continuation.

Thus, we have:

Proposition 4.2. For any hermitian toric positive line bundle over a toric variety, the Szegó kernel for the metrics $h_{\varphi}^{k}$ have the asymptotic expansions in a local frame on $M$,

$$
\Pi_{h^{k}}(z, w) \sim e^{k\left(F_{\mathbb{C}}(z \cdot \bar{w})-\left(F\left(|z|^{2}\right)+F\left(|w|^{2}\right)\right) / 2\right.} A_{k}(z, w) \quad \bmod k^{-\infty},
$$

where

$$
A_{k}(z, w) \sim k^{m}\left(a_{0}(z, w)+\frac{a_{1}(z, w)}{k}+\cdots\right)
$$

is a semiclassical symbol of order $m$.

As an example, the Bargmann-Fock(-Heisenberg) Szegó kernel with $k=1$ and $H=I$ is given (up to a constant $C_{m}$ depending only on the dimension) by

$$
\hat{\Pi}_{h_{B F}}(z, \theta, w, \varphi)=e^{z \cdot \bar{w}-\left(|z|^{2}+|w|^{2}\right) / 2} e^{i(\theta-\varphi)}=\sum_{\alpha \in \mathbb{Z}^{n}} \frac{z^{\alpha} \overline{w^{\alpha}}}{\alpha !} e^{-\left(|z|^{2}+|w|^{2}\right) / 2} e^{i(\theta-\varphi)} .
$$

The higher Szegő kernels are Heisenberg dilates of this kernel:

$$
\hat{\Pi}_{h_{B F}^{k}}(x, y)=\frac{1}{\pi^{m}} k^{m} e^{i k(t-s)} e^{k\left(\zeta \cdot \bar{\eta}-(1 / 2)|\zeta|^{2}-(1 / 2)|\eta|^{2}\right)},
$$

where $x=(\zeta, t), y=(\eta, s)$. In this case, the almost analytic extension is analytic and $F_{B F, \mathbb{C}}(z, w)=z \cdot \bar{w}$.

A second example is the Fubini-Study Szegó kernel on $\mathscr{O}(k)$, which lifts to $S^{2 m-1} \times S^{2 m-1}$ as

$$
\hat{\Pi}_{h_{\mathrm{FS}}^{k}}(x, y)=\sum_{J} \frac{(k+m) !}{\pi^{m} j_{0} ! \cdots j_{m} !} x^{J} \bar{y}^{J}=\frac{(k+m) !}{\pi^{m} k !}\langle x, y\rangle^{k} .
$$

Recalling that

$$
x=e^{i \theta} \frac{e(z)}{\|e(z)\|}
$$

in a local frame $e$ over an affine chart, the Szegó kernel has the local form on $\mathbb{C}^{m} \times \mathbb{C}^{m}$ of

$$
\hat{\Pi}_{h_{\mathrm{FS}}^{k}}(z, 0 ; w, 0)=\frac{(k+m) !}{\pi^{m} k !} \exp \left(k \log \frac{(1+z \cdot \bar{w})}{\left(\sqrt{1+|z|^{2}} \sqrt{1+|w|^{2}}\right)}\right) .
$$

Thus, $F_{\mathrm{FS}, \mathbb{C}}(z, w)=\log (1+z \cdot \bar{w})$.

4B. Asymptotics of derivatives of toric Bergman-Szegó kernels. One of the key ingredients in of Theorem 1.1 is the asymptotics of derivatives of the contracted Bergman-Szegó kernel

$$
\Pi_{h_{t}^{k}}(z, z)=F_{h_{t}^{k}}(z, z)\left\|e_{L}^{k}(z)\right\|_{h^{k}}^{2}=\hat{\Pi}_{h^{k}}(z, 0 ; z, 0)
$$

in $(t, z)$. (The notation is slightly ambiguous since in (73) it is used for the uncontracted kernel, but it is standard and we hope no confusion will arise since one is scalar-valued and the other is not.) These derivatives allow us to make simple comparisons to derivatives of $\varphi_{k}(t, z)$. Since we are ultimately interested in $C^{k}$ norms we need asymptotics of derivatives with respect to nonvanishing vector fields. 
We can use the vector fields $\left(\partial / \partial \rho_{j}\right)$ away from $\mathscr{D}$ and the vector fields $\left(\partial / \partial r_{j}\right)$ near $\mathscr{D}$. The calculations are very similar, but we carry them both out in some detail here. Later we will tend to suppress the calculations with $\left(\partial / \partial r_{j}\right)$ to avoid duplication; the reader can check in this section that the calculations and estimates are valid.

Only the leading coefficient and the order of asymptotics are relevant. The undifferentiated diagonal asymptotics are of the following form: for any $h \in P(M, \omega)$ we have

$$
\Pi_{h^{k}}(z, z)=\sum_{i=0}^{d_{k}}\left\|s_{i}(z)\right\|_{h_{k}}^{2}=a_{0} k^{m}+a_{1}(z) k^{m-1}+a_{2}(z) k^{m-2}+\cdots,
$$

where $a_{0}$ is constant and as above $d_{k}+1=\operatorname{dim} H^{0}\left(M, L^{k}\right)$.

We first consider derivatives with respect to $\rho$. Calculating $\rho$ derivatives of $\Pi_{h^{k}}\left(e^{\rho / 2}, e^{\rho / 2}\right)$ is equivalent to calculating $\theta$-derivatives of $\Pi_{h_{t}^{k}}\left(e^{i \theta} z, z\right)$. Using (62) we have

$$
\Pi_{h_{t}^{k}}\left(e^{i \theta} z, z\right)=\sum_{\alpha \in k P \cap \mathbb{Z}^{m}} \frac{e^{i\langle\alpha, \theta\rangle}\left|z^{\alpha}\right|^{2} e^{-k F_{t}\left(e^{i \theta}|z|^{2}\right)}}{2_{h_{t}^{k}}(\alpha)} .
$$

The results are globally valid but are not useful near $\mathscr{D}$ since on each stratum some of the vector fields generating the $\left(\mathbb{C}^{*}\right)^{m}$ action vanish.

Below, we use the tensor product notation $\left(\frac{\alpha}{k}-\mu_{h_{t}}\left(e^{\rho / 2}\right)\right)_{i j}^{\otimes 2}$ for $\left(\frac{\alpha_{i}}{k}-\mu_{h_{t}}\left(e^{\rho / 2}\right)_{i}\right)\left(\frac{\alpha_{j}}{k}-\mu_{h_{t}}\left(e^{\rho / 2}\right)_{j}\right)$.

Proposition 4.3. For $i, j=1, \ldots, m$ we have:

(1) $k^{-m} \sum_{\alpha \in k P \cap \mathbb{Z}^{m}}\left(\frac{\alpha}{k}-\mu_{h_{t}}\left(e^{\rho / 2}\right)\right) \frac{e^{\langle\alpha, \rho\rangle-k \varphi_{t}\left(e^{\rho / 2}\right)}}{2_{h_{t}^{k}}(\alpha)}=O\left(k^{-2}\right)$;

(2) $\frac{1}{\Pi_{h_{t}^{k}}(z, z)}\left(-\sum_{\alpha \in k P \cap \mathbb{Z}^{m}}\left(\frac{\partial}{\partial t} \log 2_{h_{t}^{k}}(\alpha)\right) \frac{e^{\langle\alpha, \rho\rangle-k \varphi_{t}\left(e^{\rho / 2}\right)}}{2_{h_{t}^{k}}(\alpha)}\right)-k \frac{\partial}{\partial t} \varphi_{t}=O\left(k^{-1}\right)$;

(3) $\frac{1}{\Pi_{h_{t}^{k}}(z, z)}\left(k^{2} \sum_{\alpha \in k P \cap \mathbb{Z}^{m}}\left(\frac{\alpha}{k}-\mu_{h_{t}}\left(e^{\rho / 2}\right)\right)_{i j}^{\otimes 2} \frac{e^{\langle\alpha, \rho\rangle-k \varphi_{t}\left(e^{\rho / 2}\right)}}{2_{h_{t}^{k}}(\alpha)}\right)-k \frac{\partial^{2} \varphi_{t}}{\partial \rho_{i} \partial \rho_{j}}=O\left(k^{-1}\right)$;

(4) $\frac{1}{\Pi_{h_{t}^{k}}(z, z)}\left(k \sum_{\alpha \in k P \cap \mathbb{Z}^{m}}\left(\frac{\alpha}{k}-\mu_{h_{t}}\left(e^{\rho / 2}\right)\right)_{i}\left(\frac{\partial}{\partial t} \log \mathscr{2}_{h_{t}^{k}}(\alpha)\right) \frac{e^{\langle\alpha, \rho\rangle-k \varphi_{t}\left(e^{\rho / 2}\right)}}{2_{h_{t}^{k}}(\alpha)}\right)-k \frac{\partial^{2} \varphi_{t}}{\partial \rho_{i} \partial t}=O\left(k^{-1}\right)$.

Proof. To prove (1), we differentiate and use (53)-(62) and (93) to obtain

$$
O\left(k^{m-1}\right)=\nabla_{\rho} \Pi_{h_{t}^{k}}\left(e^{\rho / 2}, e^{\rho / 2}\right)=k \sum_{\alpha \in k P \cap \mathbb{Z}^{m}}\left(\frac{\alpha}{k}-\mu_{h_{t}}\left(e^{\rho / 2}\right)\right) \frac{e^{\langle\alpha, \rho\rangle-k \varphi_{t}\left(e^{\rho / 2}\right)}}{2_{h_{t}^{k}}(\alpha)} .
$$

To prove (2) we differentiate

$$
\log \Pi_{h_{t}^{k}}\left(e^{\rho / 2}, e^{\rho / 2}\right)=\log \sum_{\alpha \in k P \cap \mathbb{Z}^{m}} \frac{e^{\langle\alpha, \rho\rangle-k \varphi_{t}\left(e^{\rho / 2}\right)}}{2_{h_{t}^{k}}^{k}(\alpha)}
$$

with respect $t$ to produce the left side. Since the leading coefficient of (93) is independent of $t$, the $t$ derivative has the order of magnitude of the right side of (2). 
To prove (3), we take a second derivative of (1) in $\rho$ (or $\theta$ ) to get

$$
\nabla_{\rho}^{2} \Pi_{h_{t}^{k}}\left(e^{\rho / 2}, e^{\rho / 2}\right)=-k \nabla \mu_{h_{t}}\left(e^{\rho / 2}\right) \Pi_{h_{t}^{k}}\left(e^{\rho / 2}, e^{\rho / 2}\right)+k^{2} \sum_{\alpha \in k P \cap \mathbb{Z}^{m}}\left(\frac{\alpha}{k}-\mu_{h_{t}}\left(e^{\rho / 2}\right)\right)^{\otimes 2} \frac{e^{\langle\alpha, \rho\rangle-k \varphi_{t}\left(e^{\rho / 2}\right)}}{2_{h_{t}^{k}}(\alpha)} .
$$

Then (3) follows from (93) and the fact that $\nabla \mu_{h_{t}}\left(e^{\rho / 2}\right)=\nabla^{2} \varphi$. Similar calculations show (4).

In our applications, we actually need asymptotics of logarithmic derivatives. They follow in a straightforward way from Proposition 4.3, using that $\Pi_{h^{k}}(z, z) \sim k^{m}$. We record the results for future reference.

Proposition 4.4. We have

$$
\begin{aligned}
& \frac{1}{k} \nabla_{\rho} \log \sum_{\alpha \in k P \cap \mathbb{Z}^{m}} \frac{\left|S_{\alpha}(z)\right|_{h_{t}^{k}}^{2}}{2_{h_{t}^{k}}(\alpha)}=\frac{\sum_{\alpha}\left(\frac{\alpha}{k}-\mu_{h_{t}}(z)\right) \frac{e^{\langle\alpha, \rho\rangle}}{2_{h_{t}^{k}}(\alpha)}}{\sum_{\alpha} \frac{e^{\langle\alpha, \rho\rangle}}{2_{h_{t}^{k}}(\alpha)}}=O\left(\frac{1}{k^{2}}\right), \\
& \frac{1}{k} \frac{\partial}{\partial t} \log \sum_{\alpha \in k P \cap \mathbb{Z}^{m}} \frac{\left|S_{\alpha}(z)\right|_{h_{t}^{k}}^{2}}{2_{h_{t}^{k}}(\alpha)}=\frac{\sum_{\alpha} \partial_{t} \log \left(\frac{1}{2_{h_{t}^{k}}(\alpha)}\right) \frac{e^{\langle\alpha, \rho\rangle}}{2_{h_{t}^{k}}(\alpha)}}{\sum_{\alpha} \frac{e^{\langle\alpha, \rho\rangle}}{2_{h_{t}^{k}}(\alpha)}}-\frac{\partial \varphi_{t}}{\partial t}=O\left(\frac{1}{k^{2}}\right) .
\end{aligned}
$$

Proposition 4.5. We have

$$
\begin{aligned}
& \text { (1) } \frac{1}{k} \nabla_{\rho}^{2} \log \sum_{\alpha \in k P \cap \mathbb{Z}^{m}} \frac{\left|S_{\alpha}(z)\right|_{h_{t}^{k}}^{2}}{2_{h_{t}^{k}}(\alpha)}=\frac{1}{k} \sum_{\alpha, \beta}(\alpha-\beta)^{\otimes 2} \frac{e^{\langle\alpha, \rho\rangle}}{2_{h_{t}^{k}}(\alpha)} \frac{e^{\langle\beta, \rho\rangle}}{2_{h_{t}^{k}}(\beta)}\left(\sum_{\alpha} \frac{e^{\langle\alpha, \rho\rangle}}{2_{h_{t}^{k}}(\alpha)}\right)^{-2}-\frac{\partial^{2} \varphi_{t}}{\partial \rho_{i} \partial \rho_{j}}=O\left(\frac{1}{k^{2}}\right) \text {, } \\
& \text { (2) } \frac{1}{k} \frac{\partial}{\partial t} \nabla_{\rho} \log \sum_{\alpha \in k P \cap \mathbb{Z}^{m}} \frac{\left|S_{\alpha}(z)\right|_{h_{t}^{k}}^{2}}{2_{h_{t}^{k}}(\alpha)}=\frac{1}{k} \frac{\sum_{\alpha, \beta}(\alpha-\beta) \partial_{t} \log \left(\frac{2_{h_{t}^{k}}(\beta)}{2_{h_{t}^{k}}(\alpha)}\right) \frac{e^{\langle\alpha, \rho\rangle}}{2_{h_{t}^{k}}(\alpha)} \frac{e^{\langle\beta, \rho\rangle}}{\mathscr{2}_{h_{t}^{k}}(\beta)}}{\left(\sum_{\alpha} \frac{e^{\langle\alpha, \rho\rangle}}{2_{h_{t}^{k}}(\alpha)}\right)^{2}}-\frac{\partial^{2} \varphi_{t}}{\partial \rho_{i} \partial t}=O\left(\frac{1}{k^{2}}\right) \text {, } \\
& \text { (3) } \frac{1}{k} \frac{\partial^{2}}{\partial t^{2}} \log \sum_{\alpha \in k P \cap \mathbb{Z}^{m}} \frac{\left|S_{\alpha}(z)\right|_{h_{t}^{k}}^{2}}{2_{h_{t}^{k}}(\alpha)} \\
& =\frac{1}{k} \frac{\sum_{\alpha, \beta}\left(\partial_{t}^{2} \log \frac{1}{2_{h_{t}^{k}(\alpha)}}+\left(\partial_{t} \log \frac{1}{2_{h_{t}^{k}}}\right)\left(\partial_{t} \log \frac{2_{h_{t}^{k}}(\beta)}{2_{h_{t}^{k}}(\alpha)}\right) \frac{e^{\langle\alpha, \rho\rangle}}{2_{h_{t}^{k}}(\alpha)}\right) \frac{e^{\langle\beta, \rho\rangle}}{2_{h_{t}^{k}}(\beta)}}{\left(\sum_{\alpha} \frac{e^{\langle\alpha, \rho\rangle}}{2_{h_{t}^{k}}(\alpha)}\right)^{2}}-\frac{\partial^{2} \varphi_{t}}{\partial t^{2}}=O\left(\frac{1}{k^{2}}\right) .
\end{aligned}
$$

Finally, we consider the analogous derivatives with respect to the radial coordinates $r_{j}$ near $\mathscr{D}$. We assume $z$ is close to the component of $\mathscr{D}$ given in local slice orbit coordinates by $z^{\prime}=0$ and let $r^{\prime}=\left(r_{j}\right)_{j=1}^{p}$ denote polar coordinates in this slice as discussed in Section 2. The Szegő kernel then has the form

$$
\Pi_{h_{t}^{k}}(z, z)=\sum_{\alpha \in k P \cap \mathbb{Z}^{m}} \frac{\prod_{j=1}^{p} r_{j}^{2 \alpha_{j}} e^{\left\langle\rho^{\prime \prime}, \alpha^{\prime \prime}\right\rangle} e^{-k F_{t}\left(r_{1}^{2}, \ldots, r_{p}^{2}, e^{\rho_{p+1}}, \ldots, e^{\rho_{m}}\right)}}{2_{h_{t}^{k}}(\alpha)}
$$


The coefficients of the expansion (93) are smooth functions of $r_{j}^{2}$ and the expansion may be differentiated any number of times.

The behavior of $\Pi_{h_{t}^{k}}(z, z)$ for $z \in \mathscr{D}$ has the new aspect that many of the terms vanish. The extreme case is where $z$ is a fixed point. We choose the slice coordinates so that it has coordinates $z=0$. We observe that only the term with $\alpha=0$ in (94) is nonzero, and the $\alpha$-th term vanishes to order $|\alpha|$.

Since

$$
\frac{\partial}{\partial r_{j}}=\frac{2}{r_{j}} \frac{\partial}{\partial \rho_{j}}
$$

where both are defined, the calculations above are only modified by the presence of new factors of $\frac{2}{r_{j}}$ in each space derivative. Since we are applying the derivative to functions of $r_{j}^{2}$, it is clear that the apparent poles will be canceled. Indeed, the $r_{j}$ derivative removes any lattice point $\alpha$ with vanishing $\alpha_{j}$ component. Comparing these derivatives with derivatives of (94) gives the following:

Proposition 4.6. For $n=1, \ldots, p$, we have

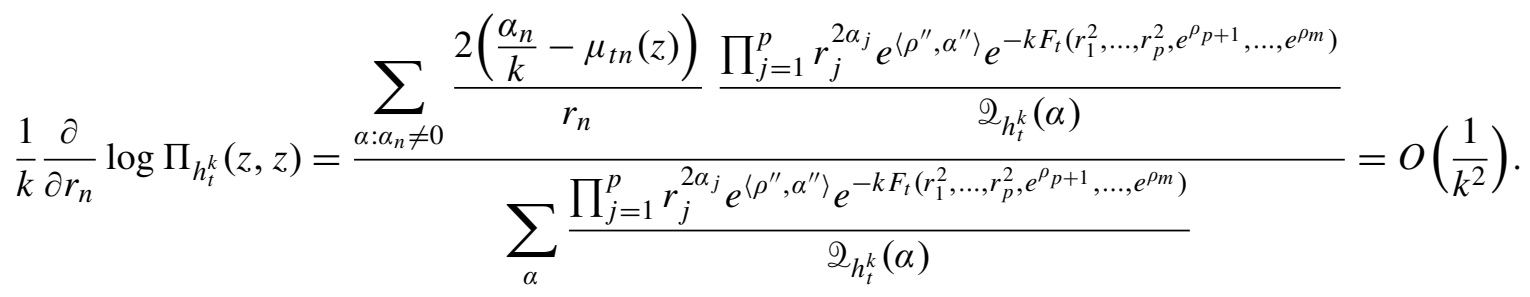

In effect, the exponent $\alpha$ is taken to $\alpha-\left(0, \ldots, 1_{n}, \ldots\right)$ in the sum or removed if $\alpha_{n}=0$, where $\left(0, \ldots, 1_{n}, \ldots\right)$ is the lattice point with only a 1 in the $n$-th coordinate. There are similar formulae for the second derivatives

$$
\frac{\partial^{2}}{\partial r_{n} \partial r_{i}}, \quad \frac{\partial^{2}}{\partial r_{n} \partial t}, \quad \frac{\partial^{2}}{\partial r_{n} \partial \rho_{i}} .
$$

The only important point to check is that the modification changing $\alpha$ to $\alpha-\left(0, \ldots, 1_{n}, \ldots\right)$ does not affect the proofs in Sections 7 and 8.

\section{Localization of sums: proof of the Localization Lemma 1.2}

The following proposition immediately implies Lemma 1.2:

Proposition 5.1. Given $(t, z)$, and for any $\delta, C>0$, there exists $C^{\prime}>0$ such that

$$
\frac{\left|s_{\alpha}(z)\right|_{h_{t}^{k}}^{2}}{\mathscr{2}_{h_{t}^{k}}(\alpha)}=\mathscr{P}_{h_{t}^{k}}(\alpha, z)=O\left(k^{-C}\right) \text { if }\left|\frac{\alpha}{k}-\mu_{h_{t}}(z)\right| \geq C^{\prime} k^{-1 / 2+\delta} .
$$

Proof. The proof is based on integration by parts. All of the essential issues occur in the Bargmann-Fock model, so we first illustrate with that case.

5A. The Bargmann-Fock case. To analyze the decay of $\mathscr{P}_{h_{B F}^{k}}(\alpha, z)$ as a function of lattice points $\alpha$, it seems simplest to use the following integral formula (suppressing the factor $k^{m}$ and normalizing the volume of $\mathbf{T}^{m}$ to equal one):

$$
k^{|\alpha|} \frac{\left|z^{\alpha}\right|^{2}}{\alpha !} e^{-k|z|^{2}}=(2 \pi)^{-m} \int_{T^{m}} e^{-k\left(\left(1-e^{i \theta}\right)|z|^{2}-i\langle\alpha / k, \theta\rangle\right)} d \theta=e^{-k|z|^{2}}(2 \pi)^{-m} \int_{T^{m}} e^{k\left(e^{i \theta}|z|^{2}-i\langle\alpha / k, \theta\rangle\right)} d \theta .
$$


Here we denote $e^{i \theta}|z|^{2}$ by $\left\langle e^{i \theta} z, z\right\rangle$ for simplicity.

The rightmost expression in (95) is $e^{-k|z|^{2}}$ times a complex oscillatory integral with phase

$$
\Phi_{z, \alpha / k}(\theta)=\left(e^{i \theta}-1\right)|z|^{2}-i\left\langle\frac{\alpha}{k}, \theta\right\rangle .
$$

We observe that (consistent with Proposition 2.1),

$$
\nabla_{\theta} \Phi_{z, \alpha / k}(\theta)=i\left(e^{i \theta}|z|^{2}-\frac{\alpha}{k}\right)=0 \Longleftrightarrow e^{i \theta}|z|^{2}=|z|^{2}=\frac{\alpha}{k} .
$$

Further, we claim that

$$
\left|\nabla_{\theta} \Phi_{z,(\alpha / k)}(\theta)\right| \geq\left.|| z\right|^{2}-\frac{\alpha}{k} \mid
$$

Indeed, the function

$$
f_{z, \alpha}(\theta):=\left.\left|e^{i \theta}\right| z\right|^{2}-\left.\frac{\alpha}{k}\right|^{2}=\sum_{j=1}^{m}\left(\cos \theta_{j}\left|z_{j}\right|^{2}-\frac{\alpha_{j}}{k}\right)^{2}+\left(\sin \theta_{j}\left|z_{j}\right|^{2}\right)^{2}
$$

on $\mathbf{T}^{m}$ has a strict global minimum at $\theta=0$ as long as $\left|z_{j}\right|^{2} \neq 0$ and $\alpha_{j} / k \neq 0$ for all $j$. We note that this discussion of global minima is possible only because the Kähler potential admits a global analytic continuation in $(z, w)$; in general, one can only analyze critical points near the diagonal.

We integrate by parts with the operator

$$
\mathscr{L}=\frac{1}{k} \frac{1}{\left|\nabla_{\theta} \Phi_{z, \alpha / k}\right|^{2}} \overline{\nabla_{\theta} \Phi_{z, \alpha / k}} \cdot \nabla_{\theta}
$$

that is, we apply its transpose

$$
\mathscr{L}^{t}=-\frac{1}{k} \frac{1}{\left|\nabla_{\theta} \Phi_{z, \alpha / k}\right|^{2}} \nabla_{\theta} \Phi_{z, \alpha / k} \cdot \nabla_{\theta}-\frac{1}{k} \nabla_{\theta} \cdot \frac{1}{\left|\nabla_{\theta} \Phi_{z, \alpha / k}\right|^{2}} \nabla_{\theta} \Phi_{z, \alpha / k}
$$

to the amplitude. The second (divergence) term is -1 times

$$
\frac{1}{k} \frac{\nabla \cdot \nabla \Phi_{z, \alpha / k}}{\left|\nabla \Phi_{z, \alpha / k}\right|^{2}}+\frac{1}{k} \frac{\left\langle\nabla^{2} \Phi_{z, \alpha / k} \cdot \nabla \Phi_{z, \alpha / k}, \nabla \Phi_{z, \alpha / k}\right\rangle}{\left|\nabla \Phi_{z, \alpha / k}\right|^{4}} .
$$

We will need to take into account the $k$-dependence of the coefficients, and therefore introduce some standard spaces of semiclassical symbols. We denote by $S_{\delta}^{n}\left(\mathbf{T}^{m}\right)$ the class of smooth functions $a_{k}(\theta)$ on $\mathbf{T}^{m} \times \mathbb{N}$ satisfying

$$
\sup _{e^{i \theta} \in \mathbf{T}^{m}}\left|D_{\theta}^{\gamma} a_{k}(\theta)\right| \leq C k^{n+|\gamma| \delta} .
$$

Here we use multiindex notation $D_{\theta}^{\gamma}=\prod_{j=1}^{m}\left(-i \partial / \partial \theta_{j}\right)^{\gamma_{j}}$. Thus, each $D_{\theta_{j}}$ derivative gives rise to an extra order of $k^{\delta}$ in estimates of $a_{k}$. We note that products of symbols satisfy

$$
S_{\delta}^{n_{1}} \times S_{\delta}^{n_{2}} \subset S_{\delta}^{n_{1}+n_{2}} .
$$

We now claim that, with $\delta$ the same as in the statement of the proposition,

$$
\frac{\nabla_{\theta} \Phi_{z, \alpha / k}}{\left|\nabla_{\theta} \Phi_{z, \alpha / k}\right|^{2}} \in S_{1 / 2-\delta}^{1 / 2-\delta}
$$

while the quantity in (99) — note in particular the prefactor $1 / k$ - lies in $S_{1 / 2-\delta}^{-2 \delta}$. 
To prove the claim, we first observe that the sup norm estimates are correct by (96) and from the fact that $\nabla \Phi_{z, \alpha / k} /\left|\nabla \Phi_{z, \alpha / k}\right|$ is a unit vector. We further consider derivatives of (101) and (96). Each $\theta$ derivative essentially introduces one more factor of $k\left|\nabla_{\theta} \Phi_{z, \alpha / k}\right|$ and hence raises the order by $k^{1 / 2-\delta}$. This continues to be true for iterated derivatives, proving the claim.

Now we observe that

$$
\mathscr{L}^{t}: S_{1 / 2-\delta}^{n} \rightarrow S_{1 / 2-\delta}^{n-2 \delta}
$$

Indeed, the first term of $\mathscr{L}^{t}$ is the composition of (i) $\nabla_{\theta}$, which raises the order by $\frac{1}{2}-\delta$, (ii) multiplication by an element of $S_{1 / 2-\delta}^{1 / 2-\delta}$, which again raises the order by $\frac{1}{2}-\delta$, and (iii) $1 / k$, which lowers the order by 1 . The second term is $1 / k$ times an element of $S_{1 / 2-\delta}^{1-2 \delta}$ and thus also lowers the order by $2 \delta$.

It follows that each partial integration by $\mathscr{L}$ introduces decay of $k^{-2 \delta}$. Hence, for any $M>0$,

$(95)=e^{-k|z|^{2}}(2 \pi)^{-m} \int_{T^{m}} e^{k\left(e^{i \theta}|z|^{2}-i\langle\alpha / k, \theta\rangle\right)}\left(\left(\mathscr{L}^{t}\right)^{M} 1\right) d \theta=O\left(k^{-2 \delta}\right)^{M} e^{-k|z|^{2}} \int_{T^{m}} e^{k \operatorname{Re}\left(e^{i \theta}|z|^{2}\right)} d \theta=O\left(k^{-2 \delta M}\right)$ in this region.

5B. General case. We now generalize this argument from the model case to the general one. With no loss of generality we may choose coordinates so that $z$ lies in a fixed compact subset of $\mathbb{C}^{m}$, where the open orbit is identified with $\left(\mathbb{C}^{*}\right)^{m}$. In the open orbit we continue to write $|z|^{2}=e^{\rho}$. The first step is to obtain a useful oscillatory integral formula for $\mathscr{P}_{h^{k}}(\alpha, z)$. By Propositions 3.3 and 4.2, we have

$$
\mathscr{P}_{h^{k}}(\alpha, z)=(2 \pi)^{-m} \int_{T^{m}} e^{k\left(F_{\mathbb{C}}\left(e^{i \theta}|z|^{2}\right)-F\left(|z|^{2}\right)\right)} \chi\left(d\left(z, e^{i \theta} z\right)\right) A_{k}\left(z, e^{i \theta} z, 0\right) e^{i\langle\alpha, \theta\rangle} d \theta+O\left(k^{-\infty}\right) .
$$

The phase is given by

$$
\Phi_{z, \alpha / k}(\theta)=F_{\mathbb{C}}\left(e^{i \theta}|z|^{2}\right)-F\left(|z|^{2}\right)-i\left\langle\frac{\alpha}{k}, \theta\right\rangle,
$$

where as above, $F_{\mathbb{C}}\left(e^{i \theta}|z|^{2}\right)$ is the almost analytic continuation of the Kähler potential $F\left(|z|^{2}\right)$ to $M \times M$. By (84) and (61), it satisfies

$$
\operatorname{Re}\left(F_{\mathbb{C}}\left(e^{i \theta}|z|^{2}\right)-F\left(|z|^{2}\right)\right) \leq-C d\left(z, e^{i \theta} z\right)^{2} \quad \text { for some } C>0 .
$$

Hence, the integrand (103) is rapidly decaying on the set of $\theta$ where $d\left(z, e^{i \theta} z\right)^{2} \geq C(\log k) / k$ (see also (86)), and we may replace $\chi\left(d\left(z, e^{i \theta} z\right)\right)$ by $\chi\left(k^{1 / 2-\delta^{\prime}} d\left(z, e^{i \theta} z\right)\right) \in S_{1 / 2-\delta^{\prime}}^{0}$, since the contribution from $1-\chi\left(k^{1 / 2-\delta^{\prime}} d\left(z, e^{i \theta} z\right)\right)$ is rapidly decaying. Here, $\delta^{\prime}$ is an arbitrarily small constant and we may choose it so that $\delta^{\prime}<\delta$ in the proposition. (We did not use such cutoffs in the Bargmann-Fock case since the real analytic potential had a global analytic extension with obvious properties, but as in Section $2 \mathrm{D}$, it is necessary for almost analytic extensions).

The set $d\left(z, e^{i \theta} z\right) \leq C\left(k^{\delta^{\prime}} / \sqrt{k}\right)$ depends strongly on the position of $z$ relative to $\mathscr{D}$, or equivalently on the position of $\mu_{h}(z)$ relative to $\partial P$. For instance, if $z$ is a fixed point then $d\left(z, e^{i \theta} z\right)=0$ for all $\theta$. However, we will not need to analyze these sets until the next section.

We now generalize the integration by parts argument. Our goal is to prove that $\mathscr{P}_{h_{t}^{k}}(\alpha, z)=O\left(k^{-C}\right)$ if $\left|\alpha / k-\mu_{h_{t}}(z)\right| \geq C k^{-1 / 2+\delta}$. Now, the gradient in $\theta$ of the phase of (103) is given by

$$
\nabla_{\theta} \Phi_{z, \alpha / k}(\theta)=\nabla_{\theta} F_{\mathbb{C}}\left(e^{i \theta}|z|^{2}\right)-i \frac{\alpha}{k}=i\left(\mu_{\mathbb{C}}\left(z, e^{i \theta} z\right)-\frac{\alpha}{k}\right)
$$


where $\mu_{\mathbb{C}}\left(z, e^{i \theta} z\right)$ is the almost analytic extension of the moment map (see Section 2D). The following lemma is obvious, but we display it to highlight the relations between the small parameters $\delta$ of the proposition and $\delta^{\prime}$ in our choice of cutoffs.

Lemma 5.2. If $\left|\frac{\alpha}{k}-\mu_{h_{t}}(z)\right| \geq C k^{-1 / 2+\delta}$ and if $d\left(z, e^{i \theta} z\right) \leq C k^{-1 / 2+\delta^{\prime}}$ with $\delta^{\prime}<\delta$, then

$$
\left|\left(\mu_{h_{t}}\left(z, e^{i \theta} z\right)-\frac{\alpha}{k}\right)\right| \geq C^{\prime} k^{-1 / 2+\delta} .
$$

Proof. By Proposition 2.1,

$$
\begin{aligned}
\left|\left(\mu_{h_{t}}\left(z, e^{i \theta} z\right)-\frac{\alpha}{k}\right)\right|^{2} & =\left|\left(\operatorname{Re} \mu_{h_{t}}\left(z, e^{i \theta} z\right)-\frac{\alpha}{k}\right)\right|^{2}+\left|\frac{1}{2} \nabla_{\theta} D\left(z, e^{i \theta} z\right)\right|^{2} \\
& \geq\left|\left(\mu_{h_{t}}(z)-\frac{\alpha}{k}\right)\right|^{2}+O\left(d\left(e^{i \theta} z, z\right)\right) .
\end{aligned}
$$

It follows that, under the assumption $\left|\frac{\alpha}{k}-\mu_{h_{t}}(z)\right| \geq C k^{-1 / 2+\delta}$ of the proposition, we may integrate by parts with the operator

$$
\mathscr{L}=\frac{1}{k}\left|\nabla_{\theta} \Phi_{z, \alpha / k}\right|^{-2} \nabla_{\theta} \Phi_{z, \alpha / k} \cdot \nabla_{\theta}
$$

The transpose $\mathscr{L}^{t}$ has the same form (98) as for the Bargmann-Fock example, the only significant change being that it is now applied to a nonconstant amplitude $A_{k}$ and to the cutoff

$$
\chi\left(k^{1 / 2-\delta^{\prime}} d\left(z, e^{i \theta} z\right)\right) \in S_{1 / 2-\delta^{\prime}}^{0}
$$

as well as to its own coefficients. Differentiations of $A_{k}$ preserve the orders of terms; the only significant change in the symbol analysis in the Bargmann-Fock case is that differentiations of $\chi\left(k^{1 / 2-\delta^{\prime}} d\left(z, e^{i \theta} z\right)\right)$ bring only improvements of order $k^{-\delta^{\prime}}$ rather than $k^{-\delta}$. However, the order still decreases by at least $2 \delta^{\prime}$ on each partial integration, and therefore repeated integration by parts again gives the estimate

$$
\left|\mathscr{P}_{h^{k}}(\alpha, z)\right|=O\left(\left(k^{-\delta^{\prime}}\right)^{M} \int_{\mathbf{T}^{m}} e^{k\left(\operatorname{Re} F\left(e^{i \theta}|z|^{2}\right)-F\left(|z|^{2}\right)\right.} d \theta\right)=O\left(\left(k^{-\delta^{\prime}}\right)^{M}\right) .
$$

Remark. It is natural to use integration by parts in this estimate since the decay in $\mu_{h_{t}}(z)-\alpha / k$ must use the imaginary part of the phase and is not a matter of being far from the center of the gaussian.

5C. Further details on the phase. For future reference (see Lemma 6.2), we Taylor expand the phase (104) in the $\theta$ variable to obtain

$$
\Phi_{z, \alpha / k}(\theta)=i\langle\mu(z)-\alpha / k, \theta\rangle+\left\langle H_{\alpha / k} \theta, \theta\right\rangle+R_{3}\left(k, e^{i \theta} \mu^{-1}\left(\frac{\alpha}{k}\right)\right),
$$


where $R_{3}=O\left(|\theta|^{3}\right)$. Here, $H_{\alpha / k}=\nabla^{2} F\left(\mu^{-1}(\alpha / k)\right)$ denotes the Hessian of $\varphi$ at $\alpha / k$ (see (57) in Section 2B). Indeed, we have

$$
\begin{aligned}
F_{\mathbb{C}}\left(e^{i \theta}|z|^{2}\right)-F\left(|z|^{2}\right) & =\int_{0}^{1} \frac{d}{d t} F_{\mathbb{C}}\left(e^{i t \theta}|z|^{2}\right) d t=\int_{0}^{1}\left\langle\nabla_{\theta} F\left(e^{i t \theta}|z|^{2}\right), i \theta\right\rangle d t \\
& \left.=\left\langle\nabla_{\rho} F\left(e^{\rho}\right)\right),(i \theta)\right\rangle+\int_{0}^{1}(t-1) \nabla_{\rho}^{2}\left(F\left(e^{i t \theta+\rho}\right)\right)(i \theta)^{2} / 2 d t \\
& =i\langle\mu(z), \theta\rangle+\nabla_{\rho}^{2}\left(F\left(e^{\rho}\right)\right)(i \theta)^{2}+R_{3}\left(k, e^{i \theta} \mu^{-1}\left(\frac{\alpha}{k}\right), \theta\right) \\
& =i\langle\mu(z), \theta\rangle+\left\langle H_{z} \theta, \theta\right\rangle+R_{3}\left(k, \theta, \mu^{-1}\left(\frac{\alpha}{k}\right)\right),
\end{aligned}
$$

in the notation (57), where $H_{z}=\nabla_{\rho}^{2} F\left(|z|^{2}\right)$ and where

$$
R_{3}(k, \theta, \rho):=\int_{0}^{1}(t-1)^{2}\left\langle\nabla_{\rho}^{3}\left(F\left(e^{i t \theta+\rho}\right)\right),(i \theta)^{3} / 3 !\right\rangle d t
$$

\section{Proof of the Regularity Lemma 1.3 and joint asymptotics of $\mathscr{P}_{h^{k}}(\alpha)$}

The first statement that $\mathscr{R}_{\infty}(t, x)$ is $C^{\infty}$ up to the boundary follows from (58),

$$
\mathscr{R}_{\infty}(t, x)=\left(\frac{\delta_{\varphi_{t}}(x) \cdot \prod_{r=1}^{d} \ell_{r}(x)}{\left(\delta_{\varphi_{0}}(x) \cdot \prod_{r=1}^{d} \ell_{r}(x)\right)^{1-t}\left(\delta_{\varphi_{1}}(x) \cdot \prod_{r=1}^{d} \ell_{r}(x)\right)^{t}}\right)^{1 / 2}=\left(\frac{\delta_{\varphi_{t}}(x)}{\delta_{\varphi_{0}}(x)^{1-t} \delta_{\varphi_{1}}(x)^{t}}\right)^{1 / 2},
$$

where the functions $\delta_{\varphi}$ are positive, bounded below by strictly positive constants, and $C^{\infty}$ up to $\partial P$.

We now consider the asymptotics of $\mathscr{R}_{k}(t, \alpha)$. We determine the asymptotics of the ratio by first determining the asymptotics of the factors of the ratio. We could use either the expression (30) in terms of norming constants $2_{h}^{k}(\alpha)$ for the dual expression in terms of $\mathscr{P}_{h^{k}}(\alpha)$ in Corollary 3.2. Each approach has its advantages and each seems of interest in the geometry of toric varieties, but for the sake of simplicity we only consider $\mathscr{P}_{h^{k}}(\alpha)$ here. In [Song and Zelditch 2007a] we take the opposite approach of focusing on the norming constants. The advantage of using $\mathscr{P}_{h^{k}}(\alpha)$ is that it may be represented by a smooth complex oscillatory integral up to the boundary, while $2_{h}^{k}(\alpha)$ are singular oscillatory integrals over $P$. A disadvantage of $\mathscr{P}_{h^{k}}(\alpha)$ is that it does not extend to a smooth function on $\bar{P}$ and has singularities on $\partial P$.

The asymptotics of $\mathscr{P}_{h^{k}}(\alpha)$ are straightforward applications of steepest descent in compact subsets of $M \backslash \mathscr{D}$ but become nonuniform at $\mathscr{D}$. To gain insight into the general problem we again consider first the Bargmann-Fock model, where by (79) we have

$$
\mathscr{P}_{h_{B F}^{k}}(\alpha)=k^{m} e^{-|\alpha|} \frac{\alpha^{\alpha}}{\alpha !}=(2 \pi)^{-m} k^{m} \int_{\mathbf{T}^{m}} e^{k\left\langle e^{i \theta}-1-i \theta, \alpha / k\right\rangle} d \theta .
$$

As observed before, the factors of $k$ cancel so "asymptotics" means asymptotics as $\alpha \rightarrow \infty$. This indicates that we do not have asymptotics when $\alpha$ ranges over a bounded set, or equivalently when $\alpha / k$ is $(C / k)$-close to a corner. On the other hand, steepest descent asymptotics applies in a coordinate $\alpha_{j}$ as long as $\alpha_{j} \rightarrow \infty$. Our aim in general is to obtain steepest descent asymptotics of $\mathscr{P}_{h^{k}}(\alpha)$ in directions far from facets and Bargmann-Fock asymptotics in directions near a facet. 
6A. Asymptotics of $\mathscr{P}_{\boldsymbol{h}^{k}}(\boldsymbol{\alpha})$. The analysis of $\mathscr{P}_{h^{k}}(\alpha)$ is closely related to the analysis of $\mathscr{P}_{h^{k}}(\alpha, z)$ in Section $5 \mathrm{~B}$, and in a sense is a continuation of it. But the arguments are now more than integrations-byparts. We obtain the asymptotics of $\mathscr{P}_{h^{k}}(\alpha)$ from the integral representation analogous to (103) (see also Proposition 4.2 and Corollary 3.4). Modulo rapidly decaying functions in $k$, we have (in the notation of Proposition 4.2):

$$
\mathscr{P}_{h^{k}}(\alpha) \sim(2 \pi)^{-m} \int_{\mathbf{T}^{m}} e^{-k\left(F_{\overparen{C}}\left(e^{i \theta} \mu_{h}^{-1}(\alpha / k)\right)-F\left(\mu_{h}^{-1}(\alpha / k)\right)\right)} A_{k}\left(e^{i \theta} \mu_{h}^{-1}(\alpha / k), \mu_{h}^{-1}(\alpha / k), 0, k\right) e^{i\langle\alpha, \theta\rangle} d \theta .
$$

This largely reduces the asymptotic calculation of $\mathscr{P}_{h^{k}}(\alpha)$ to facts about the off-diagonal asymptotics of the Szegó kernel (compare Proposition 4.2).

The integral (114) is the oscillatory integral (103) but with $z=\mu^{-1}\left(\frac{\alpha}{k}\right)$. Hence, as in (104), its phase is

$$
\Phi_{\alpha / k}(\theta)=F_{\mathbb{C}}\left(e^{i \theta} \mu^{-1}\left(\frac{\alpha}{k}\right)\right)-F\left(\mu^{-1}\left(\frac{\alpha}{k}\right)\right)-i\left\langle\frac{\alpha}{k}, \theta\right\rangle .
$$

As in (84) and (105) (but with $i$ included in as part of the phase),

$$
\operatorname{Re} \Phi_{\alpha / k}(\theta) \leq-C d\left(\mu^{-1}\left(\frac{\alpha}{k}\right), e^{i \theta} \mu^{-1}\left(\frac{\alpha}{k}\right)\right)^{2} \text { for some } C>0 .
$$

Specializing (106) to our $z=\mu^{-1}\left(\frac{\alpha}{k}\right)$, we get

$$
\nabla_{\theta} \Phi_{\alpha / k}(\theta)=\nabla_{\theta} F_{\mathbb{C}}\left(e^{i \theta} \mu^{-1}\left(\frac{\alpha}{k}\right)\right)-i \frac{\alpha}{k}=i\left(\mu_{\mathbb{C}}\left(\mu^{-1}\left(\frac{\alpha}{k}\right), e^{i \theta} \mu^{-1}\left(\frac{\alpha}{k}\right)\right)-\frac{\alpha}{k}\right) .
$$

By Proposition 2.1, the complex phase has a critical point at values of $\theta$ such that $d\left(z, e^{i \theta} z\right) \leq \delta$, and $e^{i \theta} \mu^{-1}(\alpha / k)=\mu^{-1}(\alpha / k)$. For $\alpha / k \notin \partial P$, the only critical point is therefore $\theta=0$. The phase then equals zero, and hence at the critical point the real part of the phase is at its maximum of zero.

For $\alpha / k \notin \partial P$, the critical point $\theta=0$ is nondegenerate. Specializing (109) to $z=\mu^{-1}(\alpha / k)$, we have

$$
\begin{aligned}
F_{\mathbb{C}}\left(e^{i \theta} \mu_{h}^{-1}\left(\frac{\alpha}{k}\right)\right)-F\left(\mu_{h}^{-1}\left(\frac{\alpha}{k}\right)\right) & =\int_{0}^{1} \frac{d}{d t} F_{\mathbb{C}}\left(e^{i t \theta} \mu_{h}^{-1}\left(\frac{\alpha}{k}\right)\right) d t \\
& =i\left\langle\frac{\alpha}{k}, \theta\right\rangle+i\left\langle H_{\alpha / k} \theta, \theta\right\rangle+R_{3}\left(k, \theta, \mu^{-1}\left(\frac{\alpha}{k}\right)\right),
\end{aligned}
$$

where $R_{3}$ is defined in (111). Hence,

$$
\Phi_{\alpha / k}(\theta)=\left\langle H_{\alpha / k} \theta, \theta\right\rangle+R_{3}\left(\theta, k, \mu^{-1}\left(\frac{\alpha}{k}\right)\right),
$$

and finally

$$
\mathscr{P}_{h^{k}}(\alpha) \sim(2 \pi)^{-m} \int_{\mathbf{T}^{m}} e^{-k\left\langle H_{\alpha / k} \theta, \theta\right\rangle} e^{k R_{3}\left(\theta, k, \mu^{-1}(\alpha / k)\right)} A_{k}\left(\mu_{h}^{-1}\left(\frac{\alpha}{k}\right), e^{i \theta} \mu_{h}^{-1}\left(\frac{\alpha}{k}\right), 0, k\right) d \theta .
$$

Nondegeneracy of the phase is the statement that $H_{\alpha / k}$ is a nondegenerate symmetric matrix, and this follows from strict convexity of the Kähler potential or symplectic potential, see (57). But as discussed in Section $2 \mathrm{~B}, H_{\alpha / k}$ has a kernel when $\alpha / k \in \partial P$. Hence the stationary phase expansion is nonuniform for $\alpha / k \in P$ and is not possible when $\alpha / k \in \partial P$. This explains why we need to break up the analysis into several cases, and why we cannot rely on the complex stationary phase method for all of them. 
Specializing (85) and (86), we have

$$
\left|\Pi_{h^{k}}\left(e^{i \theta} \mu_{h}^{-1}\left(\frac{\alpha}{k}\right), \mu_{h}^{-1}\left(\frac{\alpha}{k}\right)\right)\right| \leq C k^{m} e^{\left.\left.-C k d(\alpha / k), e^{i \theta} \alpha / k\right)\right)^{2}}+O\left(e^{-C \sqrt{k} d\left(z, e^{i \theta} z\right)}\right) .
$$

Hence, the integrand of (114) is negligible off the set of $\theta$ where $d\left(\mu^{-1}\left(\frac{\alpha}{k}\right), e^{i \theta} \mu^{-1}\left(\frac{\alpha}{k}\right)\right) \leq C(\log k) / \sqrt{k}$. We now observe that for $d\left(z, e^{i \theta} z\right) \leq C k^{\delta} / \sqrt{k}$,

$$
d\left(e^{i \theta} z, z\right)^{2} \sim \sum_{j}\left(1-\cos \theta_{j}\right) \ell_{j}(\mu(z)),
$$

where we sum over $j$ such that $\left|\ell_{j}(\mu(z))\right| \ll 1$ (we will make this precise in the next definition). In particular,

$$
d\left(e^{i \theta} \mu_{h}^{-1}\left(\frac{\alpha}{k}\right), \mu_{h}^{-1}\left(\frac{\alpha}{k}\right)\right)^{2} \sim \sum_{j}\left(1-\cos \theta_{j}\right) \ell_{j}\left(\frac{\alpha}{k}\right) .
$$

Indeed, both in small balls in the interior and near the boundary, the calculation is universal and hence is accurately reflected in the Bargmann-Fock model with all $H_{j}=1$, where the distance squared equals

$$
\sum_{j=1}^{m}\left|e^{i \theta_{j}} z_{j}-z_{j}\right|^{2}=2 \sum_{j=1}^{m}\left|z_{j}\right|^{2}\left(1-\cos \theta_{j}\right)=2 \sum_{j=1}^{m} \ell_{j}(\mu(z))\left(1-\cos \theta_{j}\right) .
$$

This motivates the following terminology:

Definition. Let $0<\delta_{k} \ll 1$.

- $x \in P$ is $\delta_{k}$-close to the facet $F_{j}=\left\{\ell_{j}=0\right\}$ if $\ell_{j}(x) \leq \delta_{k}$.

- $x \in P$ is $\delta_{k}$-far from the facet $F_{j}=\left\{\ell_{j}=0\right\}$ if $\ell_{j}(x) \geq \delta_{k}$.

- $x$ is a $\delta_{k}$-interior point if it is $\delta_{k}$-far from all facets.

There are $m$ possible cases according to the number of facets to which $x$ is $\delta_{k}$-close. Of course, $x$ can be $\delta_{k}$-close to at most $m$ facets, in which case it is $\delta_{k}$-close to the corner defined by the intersection of these facets. We thus define

$$
\mathscr{F}_{\delta_{k}}(x)=\left\{r:\left|\ell_{r}(x)\right|<\delta_{k}\right\}
$$

We also let

$$
\delta_{k}^{\#}(x)=\# \mathscr{F}_{\delta_{k}}(x)
$$

denote the number of $\delta_{k}$-close facets to $x$. Dual to the sets $\mathscr{F}_{\delta_{k}}$ above are the sets

$$
\mathscr{F}_{F_{i_{1}}, \ldots, F_{i r}}=\left\{x: \mathscr{F}_{\delta_{k}}(x)=\left\{i_{1}, \ldots, i_{r}\right\}\right\} .
$$

The asymptotics of $\mathscr{P}_{h^{k}}(\alpha)$ depend to the leading order on the determinant of the inverse of the Hessian of the phase of (114) (see also (103)) at $\theta=0$. This Hessian is the same as the Hessian of the Kähler potential discussed in Section 2B, and we recall that its inverse is the Hessian $G$ of the symplectic potential. Hence, the asymptotics are in terms of the determinant of $G$, which has first order poles on 
$\partial P$. This indicates that the asymptotics are not uniform up to $\partial P$. We saw this as well in the explicit example of the Bargmann-Fock case. We define

$$
\mathscr{G}_{\varphi, \delta_{k}}(x)=\left(\delta_{\varphi}(x) \cdot \prod_{j \notin \mathscr{F} \delta_{k}(x)} \ell_{j}(x)\right)^{-1},
$$

where the functions $\delta_{\varphi}$ are defined in Section 2B. When $x$ is $\delta_{k}$-far from all facets, then $\varphi_{\varphi}(x)=\operatorname{det} G_{\varphi}$; compare (58). We also define $\mathscr{P}_{h_{B F}^{k}}\left(k \ell_{j}(x)\right)$ to be the unique real analytic extension of (79) to all $x \in$ $[0, \infty)$. We then consider Bargmann-Fock type functions of type (79) adapted to the corners of our polytope $P$ :

$$
\mathscr{P}_{P, k, \delta_{k}}(x)=\prod_{j \in \mathscr{F}_{\delta_{k}}(x)} \mathscr{P}_{h_{B F}^{k}}\left(k \ell_{j}\left(\frac{\alpha}{k}\right)\right)
$$

and

$$
\tilde{\mathscr{P}}_{P, k}(x)=\prod_{j \in \mathscr{F}_{\delta_{k}}(x)} k^{-1}\left(2 \pi \ell_{j}(x)\right)^{1 / 2} \mathscr{P}_{h_{B F}^{k}}\left(k \ell_{j}(x)\right) .
$$

When we straighten out the corners by affine maps to be standard octants and separate variables $x=\left(x^{\prime}, x^{\prime \prime}\right)$ into directions near and far from $\partial P$, then $\mathscr{P}_{P, k, \delta_{k}}(x)$ is by definition a function of the near variables $x^{\prime}$ and $\mathscr{G}_{\varphi, \delta_{k}}(x)$ is by definition a function of the far variables $x^{\prime \prime}$.

The main result of this section is this:

$$
\text { Proposition 6.1. } \quad \mathscr{P}_{h^{k}}(\alpha)=C_{m} k^{m / 2} \sqrt{\operatorname{det} G_{\varphi}\left(\frac{\alpha}{k}\right)} \tilde{\mathscr{P}}_{P, k}\left(\frac{\alpha}{k}\right)\left(1+R_{k}\left(\frac{\alpha}{k}, h\right)\right) \text {, }
$$

where $R_{k}=O\left(k^{-1 / 3}\right)$ and $C_{m}$ is a positive constant depending only on $m$. The expansion is uniform in the metric $h$ and may be differentiated in the metric parameter $h$ twice with a remainder of the same order.

Equivalently, with $\delta_{k}^{\#}$ defined in (126) and by letting $\delta_{k}=k^{-2 / 3}$,

$$
\mathscr{P}_{h^{k}}(\alpha)=C_{m} k^{\left(m-\delta_{k}^{\#}(\alpha / k)\right) / 2} \sqrt{\mathscr{G}_{\varphi, \delta_{k}}\left(\frac{\alpha}{k}\right)} \mathscr{P}_{P, k, \delta_{k}}\left(\frac{\alpha}{k}\right)\left(1+R_{k}\left(\frac{\alpha}{k}, h\right)\right),
$$

where again $R_{k}=O\left(k^{-1 / 3}\right)$.

The factor $k^{\left(m-\delta_{k}^{\#}(\alpha / k)\right) / 2}$ is due to the fact that we apply complex stationary phase in $m-\delta_{k}^{\#}\left(\frac{\alpha}{k}\right)$ variables to a complex oscillatory integral with symbol of order $k^{\left(m-\delta_{k}^{\#}(\alpha / k)\right)}$.

As a check, let us consider the $m$-dimensional Bargmann-Fock case where $\delta_{k}^{\#}(\alpha / k)=r$, and with no loss of generality we will assume that the first $r$ facets are the close ones. The factor $k^{m}$ in the symbol of the Szegó kernel is then split into $k^{r}$ (absorbed in $\mathscr{P}_{P, k, \delta_{k}}$ ) and $k^{m-r}$ in the far factor. As discussed in Section 3A, the far factor should have the form

$$
k^{m-r} \prod_{j=r+1}^{m} e^{-\alpha_{j}} \frac{\alpha_{j}^{\alpha_{j}}}{\alpha_{j} !} \sim k^{m-r} \prod_{j=r+1}^{m} \alpha_{j}^{-1 / 2} .
$$

The asymptotic factor in Proposition 6.1,

$$
k^{\left(m-\delta_{k}^{\#}(\alpha / k)\right) / 2}\left(\prod_{j=r+1}^{m} \frac{k}{\alpha_{j}}\right)^{1 / 2}
$$


matches this expression. Here, and throughout the proof, we always straighten out the corner to a standard octant when doing calculations in coordinates.

Secondly, as a check on the remainder, we note that it arises from two sources. As will be seen in the proof, in far directions the stationary phase remainder has the form

$$
O\left(\frac{1}{k d((\alpha / k), \partial P)}\right)
$$

while in the near directions it has the form $O\left(k(d(\alpha / k, \partial P))^{2}\right)$. When $d(\alpha / k, \partial P) \sim k^{-2 / 3}$ the remainders match.

We break up the proof into cases according to the distance of $\alpha / k$ to the various facets as $k \rightarrow \infty$. Since we are studying joint asymptotics in $(\alpha, k), \alpha$ may change with $k$.

\section{B. Interior asymptotics.}

$\alpha / \boldsymbol{k}$ is $\delta$-far from all facets. We first consider the case where $\alpha / k$ is $\delta$-far from all facets as an introduction to the problems we face. In this case, we obtain asymptotics of the integral (114) by a complex stationary phase argument. But it is not quite standard even in this interior case. In the next section, we go on to consider the same expansion when $\delta$ depends on $k$.

Lemma 6.2. Assume that there exists $\delta>0$ such that $\ell_{j}(\alpha / k) \geq \delta$ for all $j$, that is, that $\alpha / k$ is $\delta$-far from all facets. Then there exist bounded smooth functions $A_{-j}(x)$ on $\bar{P}$ such that

$$
\mathscr{P}_{h^{k}}(\alpha) \sim C_{m} k^{m / 2} \sqrt{\operatorname{det} G_{\varphi}\left(\frac{\alpha}{k}\right)}\left(1+\frac{A_{-1}(\alpha / k)}{k}+\frac{A_{-2}(\alpha / k)}{k^{2}}+\cdots+O_{\delta}\left(k^{-M}\right)\right) .
$$

Here, $G_{\varphi}=\nabla^{2} u$ (see Section $\left.2 \mathrm{~B}\right)$ and $G_{\varphi}(\alpha / k)$ is its value at $\alpha / k$; its norm is $O\left(\delta^{-1}\right)$ and its determinant is $O\left(\delta^{-m}\right)$.

Before going into the proof, we note that the only assumption on the limit points of $\alpha / k$ is that they are $\delta$-far from facets. The lattice points $\alpha$ are implicitly allowed to vary with $k$. Asymptotics of the left side clearly depend on the asymptotics of the points $\alpha / k$, and the lemma states how they do so.

Proof. We now apply the complex stationary phase method, or more precisely its proof. The usual complex stationary phase theorem applies to exponents $k \Phi(\theta)$, where $\Phi(\theta)$ is a positive phase function with a nondegenerate critical point at $\theta=0$. In our case, the phase is also $k$-dependent since it depends on $\alpha / k$ and the asymptotics of (120) therefore depend on the asymptotics of $\alpha / k$ in the domain $d(\alpha / k, \partial P) \geq \delta$. Our stated asymptotics also depend on the behavior of $\alpha / k$ in the same way.

Although the exact statement of complex stationary phase [Hörmander 1990, Theorem 7.7.5] does not apply, the proof applies without difficulty in this region. Namely, we introduce a cutoff $\chi_{\delta}(\theta)=$ $\chi\left(\delta^{-1} \theta\right) \in C^{\infty}\left(\mathbf{T}^{m}\right)$ which is equal to 1 in a $\delta$-neighborhood of $\theta=0$ and which vanishes outside a $2 \delta$ neighborhood of $\theta=0$. We decompose the integral into its $\chi_{\delta}$ and $1-\chi_{\delta}$ parts. A standard integration by parts argument, essentially the same as in the Localization Lemma 1.2 shows that the $1-\chi_{\delta}$ term is $=O\left(\delta^{-M} k^{M}\right)$ for all $M>0$. In the $\chi_{\delta}$ part the integral may be viewed as an integral over $\mathbb{R}^{m}$ and we 
may apply the Plancherel theorem as in the standard stationary phase argument to obtain

$\mathscr{P}_{h^{k}}(\alpha) \sim \frac{C_{m}}{\sqrt{\operatorname{det}\left(k H_{\alpha / k}\right)}} \int_{\mathbb{R}^{m}} e^{-\left\langle\left(k H_{\alpha / k}\right)^{-1} \xi, \xi\right\rangle} \mathscr{F}_{\theta \rightarrow \xi}\left(e^{k R_{3}\left(\theta, k, \mu^{-1}(\alpha / k)\right)} A_{k}\left(\mu_{h}^{-1}\left(\frac{\alpha}{k}\right), e^{i \theta} \mu_{h}^{-1}\left(\frac{\alpha}{k}\right), 0\right)\right)(\xi) d \xi$,

where $\mathscr{F}_{\theta \rightarrow \xi}$ is the Fourier transform.

The stationary phase expansion [Hörmander 1990, Theorem 7.7.5] is asymptotic to

$$
\left.\left(\frac{2 \pi}{k}\right)^{m / 2} \frac{e^{(i \pi / 4) \operatorname{sgn} H_{\alpha / 4}}}{\sqrt{\left|\operatorname{det} H_{\alpha / k}\right|}} \sum_{j}^{\infty} k^{-j \mathscr{P}_{\alpha / k, j} A_{k}}\left(\mu_{h}^{-1}\left(\frac{\alpha}{k}\right), e^{i \theta} \mu_{h}^{-1}\left(\frac{\alpha}{k}\right), 0\right)\right|_{\theta=0},
$$

where

$$
\mathscr{P}_{\alpha / k, j} A_{k}(0)=\left.\sum_{\nu=\mu=j} \sum_{2 v \geq 3 \mu} \frac{i^{-j} 2^{-v}}{\mu ! \nu !}\left\langle H_{\alpha / k}^{-1} D_{\theta}, D_{\theta}\right\rangle^{\nu}\left(A_{k} R_{3}^{\mu}\right)\right|_{\theta=0} .
$$

The only change in the standard argument is that we have a family of quadratic forms $H_{\frac{\alpha}{k}}$ depending on parameters $(\alpha, k)$ rather than a fixed one. But the standard proof is valid for this modification. As in the standard proof, we expand the exponential in (133) and evaluate the terms and the remainder of the exponential factor just as in [Hörmander 1990, Theorem 7.7.5], to obtain (134), which becomes

$$
\begin{aligned}
\left.\left(\operatorname{det}\left(k^{-1} G_{\varphi}\left(\frac{\alpha}{k}\right)\right)\right)\right)\left.^{1 / 2} \sum_{j=0}^{M} k^{-j}\left(\left\langle G_{\varphi}\left(\frac{\alpha}{k}\right) D_{\theta}, D_{\theta}\right\rangle\right)^{j} \chi_{\delta} e^{k R_{3}\left(k, \theta, \mu^{-1}(\alpha / k)\right)} A_{k}\left(\mu_{h}^{-1}\left(\frac{\alpha}{k}\right), e^{i \theta} \mu_{h}^{-1}\left(\frac{\alpha}{k}\right), 0, k\right)\right|_{\theta=0} \\
\quad+O\left(k^{-M} \sup _{\theta \in \operatorname{Supp} \chi_{\delta}}\left|\left\langle G_{\varphi}\left(\frac{\alpha}{k}\right) D_{\theta}, D_{\theta}\right\rangle^{M} \chi_{\delta} e^{k R_{3}\left(k, \theta, \mu^{-1}(\alpha / k)\right)} A_{k}\left(\mu_{h}^{-1}\left(\frac{\alpha}{k}\right), e^{i \theta} \mu_{h}^{-1}\left(\frac{\alpha}{k}\right), 0, k\right)\right|\right) .
\end{aligned}
$$

Here, $G_{x}$ is the Hessian of the symplectic potential, that is, the inverse of $H_{\mu^{-1}(x)}$. (See Section 2B.) We recall that $G_{x}$ has poles $x_{j}^{-1}$ of order one when $x \in \partial P$. When $d(\alpha / k, \partial P) \geq \delta$, its norm is therefore $O\left(\delta^{-1}\right)$ and its determinant is $O\left(\delta^{-m}\right)$. Since $R_{3}$ vanishes to order 3 at the critical point, the terms of the expansion can be arranged into terms of descending order as in the standard proof. If we recall that the leading term of $S$ is $k^{m}$, we obtain the statement of Proposition 6.1 in the $\delta$-interior case.

$\alpha / \boldsymbol{k}$ is $\delta_{k}$-far from facets with $\boldsymbol{k} \delta_{\boldsymbol{k}} \rightarrow \infty$. We continue to study the complex oscillatory integral (114) but now allow $\alpha / k$ to become $\delta_{k}$-close to some facet, and obtain a stationary phase expansion (with very possibly a slow decrease in the steps) under the condition that $k \delta_{k} \rightarrow \infty$. This should be feasible since the phase $k \Phi_{\alpha / k}$ is still rapidly oscillating in this region, albeit at different rates in different directions according to the proximity of $\alpha / k$ to a particular facet. The principal complication is as follows:

- The Hessian $G_{\varphi}(\alpha / k)$ now has components which blow up like $\delta_{k}^{-1}$ near the close facets. In the stationary phase expansion, we get factors of

$$
k^{-j}\left\langle G_{\varphi}\left(\frac{\alpha}{k}\right) D_{\theta}, D_{\theta}\right\rangle^{j} A_{k}\left(\mu_{h}^{-1}\left(\frac{\alpha}{k}\right), e^{i \theta} \mu_{h}^{-1}\left(\frac{\alpha}{k}\right), 0, k\right) R_{3}\left(k, \theta, \mu_{h}^{-1}\left(\frac{\alpha}{k}\right)\right)^{\mu}
$$

both in the expansion and remainder. We must verify that these terms still are of descending order. As a guide, we note that by (95), the Bargmann-Fock phase with $\mu_{h}(z)=\alpha / k$ is given by

$$
\Phi_{B F, \alpha / k}(\theta)=\left\langle\frac{\alpha}{k}, e^{i \theta}-i \theta\right\rangle=\left\langle\cos \theta+i(\sin \theta-\theta), \frac{\alpha}{k}\right\rangle,
$$


while the amplitude is constant. In this case, the phase factors into single-variable factors and one can employ the complex stationary phase method separately to each. In the general case, we will roughly split the variables $\theta$ into two groups $\left(\theta^{\prime}, \theta^{\prime \prime}\right)$, depending on $\alpha / k$, so that the $\theta^{\prime}$ variables are paired with the small components of $\alpha / k$ while the $\theta^{\prime \prime}$ variables are paired with its large components. The complex stationary phase method applies equally to either $d \theta^{\prime}$ or $d \theta^{\prime \prime}$ integral, but the orders of the terms are determined by the proximity of $\alpha / k$ to the facets.

Lemma 6.3. Let $\left\{\delta_{k}\right\}$ be a sequence such that $k \delta_{k} \rightarrow \infty$. Assume that $\ell_{j}(\alpha / k) \geq \delta_{k}$ for all $j$, that is, that $\alpha / k$ is $\delta_{k}$ far from all facets. Then in the notation of Lemma 6.2, we have

$$
\mathscr{P}_{h^{k}}(\alpha) \sim C_{m} k^{m / 2} \sqrt{\operatorname{det} G_{\varphi}\left(\frac{\alpha}{k}\right)}\left(1+\frac{A_{-1}(\alpha / k)}{k}+\frac{A_{-2}(\alpha / k)}{k^{2}}+\cdots+\frac{A_{-M}(\alpha / k)}{k^{2}}+O\left(k \delta_{k}\right)^{-M}\right),
$$

where now

$$
A_{-j}\left(\frac{\alpha}{k}\right) \leq D \delta_{k}^{-1}=C d\left(\frac{\alpha}{k}, \partial P\right)^{-j} .
$$

Remark. One may regard this as an expansion in the semiclassical parameter $\left(k \delta_{k}\right)^{-1}=(k d(\alpha / k, \partial P))^{-1}$. Proof. We need to prove that the expansion (136) may be rearranged into terms of decreasing order and that the remainder can be made to have an arbitrarily small order $k^{-M}$ by taking sufficiently many terms.

To analyze the expansion (136), we begin with a decomposition of the inverse Hessian $G_{\alpha / k}$, that is, the Hessian of the symplectic potential, which has the form $u_{0}+g$, where $g \in C^{\infty}(\bar{P})$ and where $u_{0}$ is the canonical symplectic potential (56). We continue to fix a small $\delta>0$ as in the previous section, and consider the facets to which $\alpha / k$ is $\delta$-close. We use the affine transformation to map these $\delta$-close facets to the hyperplanes $x_{j}^{\prime}=0$. In these coordinates, we may write the symplectic potential as

$$
u_{\varphi}(x)=\sum_{j \in \mathscr{F}_{\delta_{k}}} x_{j}^{\prime} \log x_{j}^{\prime}+g(x),
$$

where the Hessian of $g$ is bounded with bounded derivatives near $\alpha / k$. The Hessian $G_{\alpha / k}$ then decomposes into the sum

$$
G_{\varphi}(x)=\sum_{j \in \mathscr{F}_{\delta_{k}}(\alpha / k)} \frac{1}{x_{j}^{\prime}} \delta_{j j}+\nabla^{2} g:=G_{\varphi}^{s}(x)+\nabla^{2} g,
$$

where $\nabla^{2} g$ is smooth up to the boundary in a neighborhood of $\mathscr{F}_{\delta_{k}}(\alpha / k)$. The notation $G_{\varphi}(x)^{s}$ refers to the "singular part" of $G_{x}$. The choice of $\delta$ is not important; we are allowing $\alpha / k$ to become $\delta_{k}$ close to some facets, and for any choice of $\delta$, the sum will include such facets.

The decomposition (138) of the inverse Hessian induces a block decomposition of the Hessian operator $\left\langle G_{\alpha / k} D_{\theta}, D_{\theta}\right\rangle$. The change of variables to $x$ above induces an affine change of the $\theta$ variables, as follows. We are using the coordinates $\left(x^{\prime}, x^{\prime \prime}\right)$ on $P$ with $x^{\prime}$ denoting the linear coordinates in the directions of the normals to the facets $\mathscr{F}_{\delta_{k}}(\alpha / k)$. The normals corresponding to $\mathscr{F}_{\delta_{k}}(\alpha / k)$ generate the isotropy algebra of the subtorus $\left(\mathbf{T}^{m}\right)^{\prime}$ fixing the near facets. We have $\mathbf{T}^{m}=\left(\mathbf{T}^{m}\right)^{\prime} \times\left(\mathbf{T}^{m}\right)^{\prime \prime}$, and denote the corresponding coordinates by $\left(\theta^{\prime}, \theta^{\prime \prime}\right)$.

The Hessian operator in these coordinates has the form

$$
\left\langle G_{\varphi}\left(\frac{\alpha}{k}\right) D_{\theta}, D_{\theta}\right\rangle=\sum_{j \in \mathscr{F}_{\delta_{k}}(\alpha / k)} \frac{k}{\alpha_{j}^{\prime}} D_{\theta_{j}^{\prime} \theta_{j}^{\prime}}^{2}+\left\langle G_{\varphi}\left(\frac{\alpha}{k}\right)^{\prime \prime} D_{\theta}, D_{\theta}\right\rangle,
$$


where the second term has bounded coefficients. Evidently, the change to the interior stationary phase expansion is entirely due to the singular part of the Hessian operator

$$
\left\langle G_{\varphi}^{s}\left(\frac{\alpha}{k}\right) D_{\theta}, D_{\theta}\right\rangle:=\sum_{j \in \mathscr{F}_{\delta_{k}}(\alpha / k)} \frac{k}{\alpha_{j}^{\prime}} D_{\theta_{j}^{\prime} \theta_{j}^{\prime}}^{2}
$$

We now consider the order of magnitude of the terms in the $j$-th term (135), which has the form

$$
\left.k^{-v}\left\langle G_{\varphi}\left(\frac{\alpha}{k}\right) D_{\theta}, D_{\theta}\right\rangle^{\nu} A_{k}\left(\mu_{h}^{-1}\left(\frac{\alpha}{k}\right), e^{i \theta} \mu_{h}^{-1}\left(\frac{\alpha}{k}\right), 0, k\right) R_{3}\left(k, \theta, \mu_{h}^{-1}\left(\frac{\alpha}{k}\right)\right)^{\mu}\right|_{\theta=0},
$$

with $v-\mu=j$ and $2 v \geq 3 \mu$. The latter constraint is evident from the fact that $R_{3}$ vanishes to order 3 .

Using (139), $\left\langle G_{\varphi}(\alpha / k) D_{\theta}, D_{\theta}\right\rangle^{\nu}$ becomes a sum of terms of which the most singular is

$$
\left\langle G_{\varphi}^{s}\left(\frac{\alpha}{k}\right) D_{\theta}, D_{\theta}\right\rangle^{\nu}:=\left(\sum_{j \in \mathscr{F}_{\delta_{k}}(\alpha / k)} \frac{k}{\alpha_{j}^{\prime}} D_{\theta_{j}^{\prime} \theta_{j}^{\prime}}^{2}\right)^{v} .
$$

We will only discuss the terms generated by this operator; the discussion is similar but simpler for the other terms. In the extreme case of $\left\langle G_{\alpha / k}^{\prime \prime} D_{\theta}, D_{\theta}\right\rangle^{\nu}$, the discussion is essentially the same as in the previous section; in particular, (141) has order $k^{-j}$.

The problem with each application of $\left\langle G_{\varphi}^{s}(\alpha / k) D_{\theta}, D_{\theta}\right\rangle$ is that it raises the order by the maximum of $k / \alpha_{j}^{\prime}$, which may be as large as $k \delta_{k}$. Although we have an overall $k^{-j}$ and constraints $v-\mu=j$, $2 v \geq 3 \mu$, it is not hard to check that these are not sufficient to produce negative exponents of $k$.

The key fact which saves the situation is that the phase $\Phi_{\alpha / k}$ and amplitude $S$ depend on $\theta$ as functions of $e^{i \theta}\left|\mu^{-1}(\alpha / k)\right|^{2}$. Although $R_{3}$ has a more complicated $\theta$-dependence, its third and higher derivatives are the same as those of $\Phi_{\alpha / k}$, and it is obvious that only these contribute to (141). Hence derivatives in $\theta$ bring in factors of $\left|\mu^{-1}(\alpha / k)\right|^{2}$ by the chain rule. Due to the behavior of the moment map near a facet, these chain rule factors cancel a square root of the blowing up factor in $G_{\varphi}(\alpha / k)$. This turns out to be sufficient for a descending series due to the power $k^{-j}$ and constraint $2 v \geq 3 \mu$.

Before giving all the details, let us consider what should be the "worst" terms of (141), that is, the ones with the least decay in $k$. Each factor of $R_{3}$ comes with a factor of $k$, so one would expect terms with large $\mu$ to be worst. The worst term will be one with a maximum $\mu$ and where a maximum number of applications on operator $\left\langle G_{\alpha / k}^{s} D_{\theta}, D_{\theta}\right\rangle^{\nu}$ is applied to the chain-rule factors $e^{i \theta}\left(\left|\mu^{-1}(\alpha / k)\right|^{2}\right)_{j}$ (the $j$-th component of this vector), obtained from an application of some $D_{\theta_{j}^{\prime}}$ to $S$ or to $R_{3}$. If instead we differentiate $S$ or $R_{3}$ again, we pull out another chain rule factor, which cancels more of the bad coefficient $k / \alpha_{j}^{\prime}$.

We now give the rigorous argument. The terms of (141) have the form

$$
k^{-v+\mu} G_{\varphi}\left(\frac{\alpha}{k}\right)^{i_{1} j_{1}} \cdots G_{\varphi}\left(\frac{\alpha}{k}\right)^{i_{\nu} j_{v}} D^{\beta_{1}} R_{3} \cdots D^{\beta_{\mu}} R_{3} D^{\beta_{\mu+1}} S
$$

where $|\beta|=2 v$ and where $D^{\beta_{q}}$ denote universal constant multiples of the multinomial differential operators $\partial^{\beta_{q}} /\left(\partial \theta^{n_{1}} \ldots \partial \theta^{n_{\beta_{q}}}\right)$, where the union of the indices agrees with $\left\{i_{1}, j_{1}, \ldots, i_{v}, j_{v}\right\}$. We need each $\left|\beta_{q}\right| \geq 3$ for $q \leq \mu$ to remove the zero of $R_{3}$. If we only consider the most singular term, then we need 
$i_{q}=j_{q} \in \mathscr{F}_{\delta_{k}}(\alpha / k)$. In this case our term becomes

$$
k^{-v+\mu}\left(\prod_{\substack{1 \leq j \leq v \\ q_{j} \in \mathscr{F}_{\delta}(\alpha / k)}} \frac{k}{\alpha_{q_{j}}^{\prime}}\right) D^{\beta_{1}} R_{3} \cdots D^{\beta_{\mu}} R_{3} D^{\beta_{\mu+1}} S
$$

For each factor $k / \alpha_{q_{j}}^{\prime}$, there exist two factors of the associated differential operator $\partial / \partial \theta_{q_{j}}$. When one is applied to either $R_{3}$ or $S$ it pulls out a chain rule factor $\left.e^{i \theta_{q_{j}}} \mid \mu^{-1}(\alpha / k)\right)\left._{j}\right|^{2}$. If the second derivative is applied to this factor, it will not introduce any new factors of $\left.\mid \mu^{-1}(\alpha / k)\right)\left._{j}\right|^{2}$. We now estimate (143) by

$$
|(143)| \leq k^{-v+\mu}\left(\prod_{\substack{1 \leq j \leq v \\ q_{j} \in \mathscr{F}_{\delta}(\alpha / k)}} \frac{k}{\alpha_{q_{j}}^{\prime}}\right) \prod_{j=1}^{\mu}\left|\mu^{-1}\left(\frac{\alpha}{k}\right)_{q_{j}}\right|^{2} .
$$

Now $\mu^{-1}(x)=\nabla u_{\varphi}(x)$ in $\rho$ coordinates. So the square of the $q_{j}$-th component of $\mu^{-1}(\alpha / k)$ equals $\log \left(\alpha_{q_{j}} / k\right)$ plus a bounded remainder in $\rho$ coordinates; here as above we are using the $x_{j}$ coordinates adapted to $\alpha / k$. It follows that in the $z$ coordinates adapted to the facets of $\mathscr{D}$ corresponding to the hyperplanes $x_{j}^{\prime}=0$, with $\left|z_{j}\right|^{2}=e^{\rho_{j}}$, we have $\left|\mu^{-1}(\alpha / k)_{q_{j}}\right|^{2} \leq C \alpha_{j} / k$. The constant $C$ comes from the smooth part of the symplectic potential and has a uniform bound. As a check, we note that for the approximating Bargmann-Fock model we have $\left|z_{j}\right|^{2}=\alpha_{j} / k$. It follows from (144) and $k / \alpha_{j}^{\prime} \leq C d(\alpha / k, \partial P)^{-1}$ that

$$
|(143)| \leq C k^{-v+\mu}\left(\prod_{\substack{1 \leq j \leq v \\ q_{j} \in \mathscr{F}_{\delta}(\alpha / k)}} \frac{k}{\alpha_{q_{j}}^{\prime}}\right) \prod_{j=1}^{\mu} \frac{k}{\alpha_{q_{j}}^{\prime}} \leq C k^{-v+\mu} d\left(\frac{\alpha}{k}, \partial P\right)^{-v+\mu}=C\left(k d\left(\frac{\alpha}{k}, \partial P\right)\right)^{-j} .
$$

Effectively, the "semiclassical parameter" has changed from $k^{-1}$ to $k^{-1} d(\alpha / k, \partial P)^{-1}$, a natural parameter in boundary problems. As long as $k d(\alpha / k, \partial P) \rightarrow \infty$ at some fixed rate, we obtain a descending expansion.

6C. Boundary zones: corner zone. Having dealt with the case where $\left|\alpha_{j} / k\right| \geq \delta_{k}$, we now turn to the complementary cases where $d(\mu(z), \partial P) \leq \delta_{k}$, that is, at least for one $j,\left|\alpha_{j} / k\right| \leq \delta_{k}$ or equivalently, $\alpha / k$ is $\delta_{k}$-close to at least one facet. The choice of the scale $\delta_{k}$ is so that it is small enough to justify the Bargmann-Fock approximation in the "near" variables.

In this section, we consider the extreme corner case where $\mu(z)$ lies in a $\delta_{k}$-corner, that is, where there exists a vertex $v \in \partial P$ so that $d(\mu(z), v) \leq \delta_{k}$. Putting $v=0$, the assumption becomes that $|\mu(z)| \leq C \delta_{k}$. Our main object is to determine the scale $\delta_{k}$ so that the Bargmann-Fock approximation is valid. That is, for $z=\mu^{-1}(\alpha / k)$ we should have, in the multiindex notation of Section 2F (see (113)),

$$
\mathscr{P}_{h^{k}}(\alpha) \sim \mathscr{P}_{h_{B F}^{k}}(\alpha)=k^{m}(2 \pi)^{-m} \int_{\mathbf{T}^{m}} \exp \left(-k \sum_{j=1}^{m} H_{j \bar{j}}\left\langle e^{i \theta_{j}}-1+i \theta_{j}, \frac{\alpha_{j}}{k}\right\rangle\right) d \theta .
$$

Lemma 6.4. If $\mu(z)$ lies in a $\delta_{k}$-corner, then

$$
\mathscr{P}_{h^{k}}(\alpha)=C_{m} \mathscr{P}_{h_{B F}^{k}}(\alpha)\left(1+O\left(\delta_{k}\right)+O\left(k \delta_{k}^{2}\right)\right)=C_{m} \mathscr{P}_{h_{B F}^{k}}(\alpha)\left(1+O\left(k \delta_{k}^{2}\right)\right) .
$$


Proof. We may assume that $v=0$ and that the corner is a standard octant. The phase is

$$
k\left(F_{\mathbb{C}}\left(|z|^{2} e^{i \theta}\right)-F\left(|z|^{2}\right)-\left\langle\frac{\alpha}{k}, \theta\right\rangle\right) .
$$

We Taylor expand $F(w)$ at $w=0$ :

$$
F_{\mathbb{C}}\left(e^{i \theta}|z|^{2}\right)=F(0)+F^{\prime}(0) e^{i \theta}|z|^{2}+O\left(|z|^{4}\right),
$$

so that

$$
\left.F_{\mathbb{C}}\left(|z|^{2} e^{i \theta}\right)-F\left(|z|^{2}\right)=F^{\prime}(0)|z|^{2}\left(e^{i \theta}-1\right)\right)+O\left(|z|^{4}\right) .
$$

Since $|z|^{2}=O\left(\delta_{k}\right)$, we see that $k$ times the quartic remainder is $O\left(k \delta_{k}^{2}\right)=o(1)$ as long as $\delta_{k}=o(1 / \sqrt{k})$. Hence this part of the exponential is a symbol of order zero and may be absorbed into the amplitude. Further we note that $F^{\prime}(0)|z|^{2}=\mu(z)+O\left(|z|^{4}\right)$ and therefore we have

$$
\left.k\left(F_{\mathbb{C}}\left(|z|^{2} e^{i \theta}\right)-F\left(|z|^{2}\right)-i\left\langle\frac{\alpha}{k}, \theta\right\rangle\right)=k \mu(z)((1-\cos \theta)+i(\sin \theta-\theta))+O\left(|z|^{4}\right)\right) .
$$

It follows that when $\mu(z)=\alpha / k=O\left(\delta_{k}\right)$, the phase equals

$$
\alpha((1-\cos \theta)+i(\sin \theta-\theta))+O\left(k \delta_{k}^{2}\right) .
$$

Absorbing the $e^{O\left(k \delta_{k}^{2}\right)}=1+O\left(k \delta_{k}^{2}\right)$ term into the amplitude produces an oscillatory integral with the same phase function as for the Bargmann-Fock kernel.

Now let us consider the amplitude of the integral. We continue with the notation of Proposition 4.2. The amplitude has a semiclassical expansion $A_{k}(z, w) \sim k^{m} a_{0}(z, w)+k^{m-1} a_{1}(z, w)+\cdots$. Further, the $\mathbf{T}^{m}$-invariance implies that $A_{k}\left(e^{i \theta} z, e^{i \theta} w\right)=A_{k}(z, w)$. The leading order amplitude equals 1 when $z=w$ and thus

$$
a_{0}\left(z, e^{i \theta} w\right)=1+C e^{i \theta}|z|^{2}+O\left(|z|^{4}\right),
$$

hence the full symbol satisfies

$$
A_{k}\left(z, e^{i \theta} z\right)=k^{m}\left(1+C e^{i \theta}|z|^{2}+\cdots\right)+O\left(\delta_{k}^{2}\right) .
$$

When $\mu(z)=\alpha / k=O\left(\delta_{k}\right)$ we thus have

$$
A_{k}\left(z, e^{i \theta} z\right)=k^{m}\left(1+C e^{i \theta} \frac{\alpha}{k}+O\left(\delta_{k}^{2}\right)\right) .
$$

Therefore, $\mathscr{P}_{h^{k}}(\alpha)=\mathscr{P}_{h_{B F}^{k}}(\alpha)\left(1+O\left(\delta_{k}\right)+O\left(k \delta_{k}^{2}\right)\right)$ in the corner region.

6D. Boundary zones: mixed boundary zone. Now consider the general case where $d(\mu(z), \partial P) \leq \delta_{k}$, but $\mu(z)$ is not necessarily in a corner. Thus, at least one component $\alpha_{j} / k=O\left(\delta_{k}\right)$ but not all components need to satisfy this condition. We refer to this case as mixed since some components are small and some are not.

The basic idea to handle this case is to split the components into near and far parts, to use Taylor expansions and Bargmann-Fock approximations in the near components, and to use complex stationary phase in the far components. By Section 6B, complex stationary phase works for any sequence $\delta_{k}$ satisfying $k \delta_{k} \rightarrow \infty$, and by Section 6C the Taylor-Bargmann-Fock approximation works whenever $\delta_{k}=o(1 / \sqrt{k})$, so we have some flexibility in choosing $\delta_{k}$. 
Remark. In fact, we see that both the complex stationary phase and the Bargmann-Fock approximations are valid for $k$ satisfying (for instance)

$$
\frac{C \log k}{k} \leq \delta_{k} \leq C^{\prime} \frac{1}{\sqrt{k} \log k},
$$

although the remainder estimates will not be equally sharp by both methods. In fact, the stationary phase remainder is of order $\left(k \delta_{k}\right)^{-1}$ while the Bargmann-Fock remainder is of order $k \delta_{k}^{2}$; the two remainders agree when $\delta_{k}=k^{-2 / 3}$ and for small $\delta_{k}$ the Bargmann-Fock remainder is smaller.

We first choose linear coordinates so that $\mu(z)=\alpha / k$ is $\delta_{k}^{\prime}$ close to the first $r$ facets and $\delta_{k}^{\prime}$ far from the $p:=m-r$ remaining facets, and by an affine map we position the first $r$ facets as the first $r$ coordinate hyperplanes at $x=0$, and the remaining facets as the remaining coordinate hyperplanes. We use coordinates $\left(x^{\prime}, x^{\prime \prime}\right)$ relative to this splitting. We also write the $z$ variables as $\left(z^{\prime}, z^{\prime \prime}\right)$ in the corresponding slice-orbit coordinates and $\left(\theta^{\prime}, \theta^{\prime \prime}\right)$ as the associated coordinates on $\mathbf{T}^{m}$.

We now introduce two small scales, a smaller one $\delta_{k}^{\prime}$ to define the nearest facets, and a larger one $\delta_{k}^{\prime \prime}$. The Bargmann-Fock approximation will be used in the $x^{\prime}$ variables which are $\delta_{k}^{\prime}$ close to a facet. It is sometimes advantageous to use the Bargmann-Fock approximation in the $x^{\prime \prime}$ variables which are $\delta_{k}^{\prime \prime}$ small, but the complex phase method is also applicable. In the following, we continue to use the notation above Proposition 6.1.

Lemma 6.5. Assume $\mu(z)$ lies in the mixed boundary zone $\left\{\left|x^{\prime}\right| \leq \delta_{k}^{\prime},\left|x^{\prime \prime}\right| \leq \delta_{k}^{\prime \prime}\right\}$. If

$$
\eta_{k}=k^{-1}\left(\delta_{k}^{\prime \prime}\right)^{-1}+k\left(\delta_{k}^{\prime}\right)^{2}+k\left(\delta_{k}^{\prime}\right)^{2} \delta_{k}^{\prime \prime}+\delta_{k}^{\prime} \rightarrow 0,
$$

then $\mathscr{P}_{h^{k}}(\alpha)$ has an asymptotic expansion

$$
\mathscr{P}_{h^{k}}(\alpha)=C_{m} k^{m-p / 2} \sqrt{\mathscr{G}_{\varphi, \delta_{k}}\left(\frac{\alpha}{k}\right)} \mathscr{P}_{P, k, \delta_{k}^{\prime}}(\alpha)\left(1+O\left(\eta_{k}\right)\right) .
$$

Our strategy for obtaining asymptotics of $\mathscr{P}_{h^{k}}(\alpha)$ in this case is as follows:

- We employ steepest descent in the $p$ directions which are $\delta_{k}^{\prime \prime}$-far from all facets, that is, in the $x^{\prime \prime}$ variables. This removes the $x^{\prime \prime}$ variables and produces an expansion analogous to that of Lemma 6.2.

- In the remaining $x^{\prime}$ variables, we Taylor expand the phase and amplitude in the directions $\delta_{k}$-close to $\partial P$ as in Section $6 \mathrm{C}$.

- We thus obtain universal asymptotics to leading order depending only on the number of facets to which $\alpha / k$ is $\delta_{k}$-close.

Proof. We are still working on the oscillatory integral with phase (114), but we now treat it as an iterated complex oscillatory integral in the variables $\left(\theta^{\prime}, \theta^{\prime \prime}\right)$ defined above. We first consider the $d \theta^{\prime \prime}$ integral,

$I_{k}\left(\theta^{\prime}, \frac{\alpha}{k}\right):=(2 \pi)^{-p} \int_{\mathbf{T}^{p}} e^{k\left(F_{\complement}\left(e^{i \theta} \mu_{h}^{-1}(\alpha / k)\right)-F\left(\mu_{h}^{-1}(\alpha / k)\right)\right)} A_{k}\left(e^{i \theta} \mu_{h}^{-1}\left(\frac{\alpha}{k}\right), \mu_{h}^{-1}\left(\frac{\alpha}{k}\right), 0, k\right) e^{-i\langle\alpha, \theta\rangle} d \theta^{\prime \prime}$,

where $p$ is the number of $\theta^{\prime \prime}$ variables. We also let $r=m-p$ be the number of $\theta^{\prime}$ variables. We now verify that we may apply the complex stationary phase method to the $d \theta^{\prime \prime}$ integral for fixed $\theta^{\prime}$. Throughout this section, we put $z=\mu^{-1}(\alpha / k)$ and often write $\left|z^{\prime}\right|^{2},\left|z^{\prime \prime}\right|^{2}$ for the modulus square of the associated complex coordinate components of this point in the open orbit. 
First we simplify the complex phase. As in Section $6 \mathrm{C}$, we Taylor expand $F_{\mathbb{C}}\left(e^{i \theta^{\prime}}\left|z^{\prime}\right|^{2}, e^{i \theta^{\prime \prime}}\left|z^{\prime \prime}\right|^{2}\right)$ in the $z^{\prime}$ variable (and only in the $z^{\prime}$ variable) to obtain

$$
F_{\mathbb{C}}\left(e^{i \theta^{\prime}}\left|z^{\prime}\right|^{2}, e^{i \theta^{\prime \prime}}\left|z^{\prime \prime}\right|^{2}\right)=F_{\mathbb{C}}\left(0, e^{i \theta^{\prime \prime}}\left|z^{\prime \prime}\right|^{2}\right)+F_{1}^{\prime}\left(0, e^{i \theta^{\prime \prime}}\left|z^{\prime \prime}\right|^{2}\right) e^{i \theta^{\prime}}\left|z^{\prime}\right|^{2}+O\left(\left|z^{\prime}\right|^{4}\right),
$$

where $F_{1}$ is the $z^{\prime}$-derivative of $F$. The phase is then

$$
\begin{array}{r}
k\left(F_{\mathbb{C}}\left(e^{i \theta^{\prime}}\left|z^{\prime}\right|^{2}, e^{i \theta^{\prime \prime}}\left|z^{\prime \prime}\right|^{2}\right)-F\left(\left|z^{\prime}\right|^{2},\left|z^{\prime \prime}\right|^{2}\right)-i\left\langle\frac{\alpha^{\prime}}{k}, \theta^{\prime}\right\rangle-i\left\langle\frac{\alpha^{\prime \prime}}{k}, \theta^{\prime \prime}\right\rangle\right) \\
=k\left(F_{\mathbb{C}}\left(0, e^{i \theta^{\prime \prime}}\left|z^{\prime \prime}\right|^{2}\right)-F\left(0,\left|z^{\prime \prime}\right|^{2}\right)\right)+k\left(F_{1}^{\prime}\left(0, e^{i \theta^{\prime \prime}}\left|z^{\prime \prime}\right|^{2}\right) e^{i \theta^{\prime}}\left|z^{\prime}\right|^{2}-F_{1}^{\prime}\left(0,\left|z^{\prime \prime}\right|^{2}\right)\left|z^{\prime}\right|^{2}\right) \\
-k\left(i\left\langle\frac{\alpha^{\prime}}{k}, \theta^{\prime}\right\rangle+i\left\langle\frac{\alpha^{\prime \prime}}{k}, \theta^{\prime \prime}\right\rangle\right)+O\left(k\left|z^{\prime}\right|^{4}\right) .
\end{array}
$$

We now absorb the exponentials of the terms $k O\left(\left|z^{\prime}\right|^{4}\right), k i\left\langle\alpha^{\prime} / k, \theta^{\prime}\right\rangle$ of the phase (149) into the amplitude, that is, we take the new amplitude $A_{k}^{\prime \prime}$ to be the old one $A_{k}$ multiplied by this factor. The term $k O\left(\left|z^{\prime}\right|^{4}\right)$ is $o(1)$, while $k i\left\langle\alpha^{\prime} / k, \theta^{\prime}\right\rangle$ is constant in $\theta^{\prime \prime}$, so their exponentials are symbols in $\theta^{\prime \prime}$ and may be absorbed into the amplitude. Moreover, the term $-F_{1}^{\prime}\left(0,\left|z^{\prime \prime}\right|^{2}\right)\left|z^{\prime}\right|^{2}$ is independent of $\theta^{\prime \prime}$ so its exponential may also be absorbed into the amplitude.

The phase function for the $d \theta^{\prime \prime}$ integral thus simplifies to

$$
k\left(F_{\mathbb{C}}\left(0, e^{i \theta^{\prime \prime}}\left|z^{\prime \prime}\right|^{2}\right)-F\left(0,\left|z^{\prime \prime}\right|^{2}\right)\right)+k\left(F_{1}^{\prime}\left(0, e^{i \theta^{\prime \prime}}\left|z^{\prime \prime}\right|^{2}\right) e^{i \theta^{\prime}}\left|z^{\prime}\right|^{2}\right)-k i\left\langle\frac{\alpha^{\prime \prime}}{k}, \theta^{\prime \prime}\right\rangle .
$$

Due to the presence of $\left|z^{\prime}\right|^{2}$, the terms $k\left(F_{1}^{\prime}\left(0, e^{i \theta^{\prime \prime}}\left|z^{\prime \prime}\right|^{2}\right) e^{i \theta^{\prime}}\left|z^{\prime}\right|^{2}-F_{1}^{\prime}\left(0,\left|z_{2}\right|^{2}\right)\left|z^{\prime}\right|^{2}\right)$ are $O\left(k \delta^{\prime}\right)$, hence of much lower order than the remaining terms. To simplify the phase further, we now argue that their exponentials can also be absorbed into the amplitude, albeit as exponentially growing rather than polynomially growing factors in $k$. Since $F_{1}^{\prime}\left(0,\left|z_{2}\right|^{2}\right)\left|z^{\prime}\right|^{2}$ is independent of $\theta^{\prime \prime}$, it can be factored out of the $\theta^{\prime \prime}$ integral, so the key factor is

$$
E_{k}\left(\theta^{\prime \prime}\right):=e^{k\left(F_{1}^{\prime}\left(0, e^{i \theta^{\prime \prime}}\left|z^{\prime \prime}\right|^{2}\right) e^{i \theta^{\prime}}\left|z^{\prime}\right|^{2}\right)}
$$

where in the notation for $E_{k}$ we omit its dependence on the parameters $\left|z^{\prime \prime}\right|^{2},\left|z^{\prime}\right|^{2}, \theta^{\prime}$. Thus we would like to show that complex stationary phase method applies to the complex oscillatory integral with phase

$$
\Phi^{\prime \prime}\left(\theta^{\prime \prime}\right):=F_{\mathbb{C}}\left(0, e^{i \theta^{\prime \prime}}\left|z^{\prime \prime}\right|^{2}\right)-F\left(0,\left|z^{\prime \prime}\right|^{2}\right)-i\left\langle\frac{\alpha^{\prime \prime}}{k}, \theta^{\prime \prime}\right\rangle
$$

and with the amplitude $A_{k}^{\prime \prime}\left(\theta^{\prime \prime}\right)$ given by the original amplitude $A_{k}$ multiplied by

$$
\exp k\left(F_{1}^{\prime}\left(0, e^{i \theta^{\prime \prime}}\left|z^{\prime \prime}\right|^{2}\right) e^{i \theta^{\prime}}\left|z^{\prime}\right|^{2}-F_{1}^{\prime}\left(0,\left|z^{\prime \prime}\right|^{2}\right)\left|z^{\prime}\right|^{2}+i\left\langle\frac{\alpha^{\prime \prime}}{k}, \theta^{\prime \prime}\right\rangle+O\left(\left|z^{\prime}\right|^{4}\right)\right.
$$

The amplitude is of exponential growth but its growth is of strictly lower exponential growth than the phase factor.

The next (not very important) observation is that by (116), the real part of complex phase damps the integral so that the integrand is negligible on the complement of the set

$$
\left|\theta^{\prime \prime}\right| \leq C \frac{\delta^{\prime}}{d^{\prime \prime}(\mu(z), \partial P)}
$$


modulo rapidly decaying errors. This follows by splitting up the sum in (122)-(123) into the close facets to $z$ and the far facets. The integrand is negligible unless $|\operatorname{Re} \Phi| \leq C(\log k) / k$; hence it is negligible unless

$$
\begin{aligned}
d\left(e^{i \theta} z, z\right)^{2} & \sim \sum_{j \in \mathscr{F}_{\delta_{k}}(\mu(z))}\left(1-\cos \theta_{j}^{\prime \prime}\right) \ell_{j}^{\prime \prime}(\mu(z))+O\left(\left|z^{\prime}\right|^{2}\right) \\
& \sim \sum_{j \in \mathscr{F}_{\delta_{k}}(\mu(z))}\left(\theta_{j}^{\prime \prime}\right)^{2} \ell_{j}^{\prime \prime}(\mu(z))+O\left(\delta_{k}^{\prime}\right) \\
& \leq C \frac{\log k}{k} \Longleftrightarrow \theta_{j}^{2} \leq \frac{O\left(\delta_{k}^{\prime}\right)+O\left(\frac{\log k}{k}\right)}{d^{\prime \prime}(\mu(z), \partial P)}, \quad \text { for all } j \in \mathscr{F}_{\delta_{k}}(\mu(z)) .
\end{aligned}
$$

Under the assumption that $d^{\prime \prime}(\mu(z), \partial P) \geq \delta_{k}^{\prime \prime}$, the integrand is rapidly decaying unless $\theta_{j}^{2} \leq C \delta_{k}^{\prime} / \delta_{k}^{\prime \prime}$. We could introduce a cutoff of the form

$$
\chi\left(\sqrt{\frac{\delta_{k}^{\prime \prime}}{\delta_{k}^{\prime}}} \theta\right)
$$

but for our purposes, it suffices to use a smooth cutoff $\chi_{\delta}\left(\theta^{\prime \prime}\right)$ around $\theta^{\prime \prime}=0$ with a fixed small $\delta$ so that we may use local $\theta^{\prime \prime}$ coordinates. We then break up the integral using $1=\chi_{\delta}+\left(1-\chi_{\delta}\right)$. The $\left(1-\chi_{\delta}\right)$ term is rapidly decaying and may be neglected.

We observe that $\nabla_{\theta^{\prime \prime}} F_{\mathbb{C}}\left(0, e^{i \theta^{\prime \prime}}\left|z^{\prime \prime}\right|^{2}\right)=i \mu_{\mathbb{C}}^{\prime \prime}\left(\left|z^{\prime \prime}\right|, e^{i \theta^{\prime \prime}}\left|z^{\prime \prime}\right|\right)$ is the complexified moment map for the subtoric variety $z^{\prime}=0$, and we can use Proposition 2.1 to see that its only critical point in the domain of integration is at $\theta^{\prime \prime}=0$. We denote the Hessian of the phase (152) at $\theta^{\prime \prime}=0$ by

$$
H_{\left|z^{\prime \prime}\right|^{2}}^{\prime \prime}=\left.\nabla_{\theta^{\prime \prime}}^{2} \Phi^{\prime \prime}\left(\theta^{\prime \prime}\right)\right|_{\theta^{\prime \prime}=0}=\left.\nabla_{\theta^{\prime \prime}}^{2} F_{\mathbb{C}}\left(0, e^{i \theta^{\prime \prime}}\left|z^{\prime \prime}\right|^{2}\right)\right|_{\theta^{\prime \prime}=0},
$$

and observe that it equals $i D \mu_{\mathbb{C}}^{\prime \prime}\left(\left|z^{\prime \prime}\right|, e^{i \theta^{\prime \prime}}\left|z^{\prime \prime}\right|\right)$, the derivative of the moment map from the subtoric variety to its polytope. By the same calculation that led to (138), the $\theta^{\prime \prime}-\theta^{\prime \prime}$ block of the inverse Hessian operator has the form

$$
G_{\varphi}^{\prime \prime}\left(x^{\prime \prime}\right)=\sum_{j=1}^{p} \frac{1}{x_{j}^{\prime \prime}} \delta_{j j}+\nabla^{2} g:=\left(G_{\varphi}^{\prime \prime}\right)^{s}\left(x^{\prime \prime}\right)+\nabla^{2} g
$$

where $\left|x^{\prime \prime}\right| \geq \delta_{k}^{\prime \prime}$.

We now must verify that the complex stationary phase expansion

$$
\left.\left(\operatorname{det} k^{-1} G_{\varphi}^{\prime \prime}\left(\left|z^{\prime \prime}\right|^{2}\right)\right)^{1 / 2} \sum_{j=1}^{M} k^{-j}\left(\left\langle G_{\varphi}^{\prime \prime}\left(\left|z^{\prime \prime}\right|^{2}\right) D_{\theta^{\prime \prime}}, D_{\theta^{\prime \prime}}\right\rangle\right)^{j} \chi_{\delta} A_{k}^{\prime \prime}\left(\theta^{\prime \prime}\right)\right|_{\theta^{\prime \prime}=0}
$$

is a descending expansion in well-defined steps and that the remainder

$$
k^{-M} \sup _{\theta^{\prime \prime} \in \operatorname{Supp} \chi_{\delta}}\left|\left\langle G_{\varphi}^{\prime \prime}\left(\left|z^{\prime \prime}\right|^{2}\right) D_{\theta^{\prime \prime}}, D_{\theta^{\prime \prime}}\right\rangle^{M} A_{k}^{\prime \prime}\left(\theta^{\prime \prime}\right)^{M} \chi_{\delta} A_{k}^{\prime \prime}\left(\theta^{\prime \prime}\right)\right|
$$

is of arbitrarily small order as $M$ increases. 
We first note that the Hessian operator $k^{-1}\left\langle G_{\varphi}^{\prime \prime}\left(\left|z^{\prime \prime}\right|^{2}\right) D_{\theta^{\prime \prime}}, D_{\theta^{\prime \prime}}\right\rangle$ brings in a net order of $k^{-1}\left(\delta_{k}^{\prime \prime}\right)^{-1}$, since the coefficients $1 / x^{\prime \prime}$ in the singular part are bounded by $\left(\delta_{k}^{\prime \prime}\right)^{-1}$. The maximal order terms arise from applying the Hessian operator to the factor $E_{k}$. Each derivative can bring down a factor of

$$
\left.k F_{1}^{\prime}\left(0, e^{i \theta^{\prime \prime}}\left|z^{\prime \prime}\right|^{2}\right) e^{i \theta^{\prime}}\left|z^{\prime}\right|^{2}\right)=O\left(k \delta_{k}^{\prime} \delta_{k}^{\prime \prime}\right)
$$

Since there are two $\theta^{\prime \prime}$ derivatives for each $k^{-1}\left(\delta_{k}^{\prime \prime}\right)^{-1}$ the maximum order in $k$ from a single factor of $k^{-1}\left\langle G_{\varphi}^{\prime \prime}\left(\left|z^{\prime \prime}\right|^{2}\right) D_{\theta^{\prime \prime}}, D_{\theta^{\prime \prime}}\right\rangle$ applied to $A_{k}^{\prime \prime}$ is of order

$$
\eta_{k}=k^{-1}\left(\delta_{k}^{\prime \prime}\right)^{-1}\left(\left(k \delta_{k}^{\prime}\right)^{2}\left(\delta_{k}^{\prime \prime}\right)^{2}+k \delta_{k}^{\prime} \delta_{k}^{\prime \prime}\right)=k\left(\delta_{k}^{\prime}\right)^{2} \delta_{k}^{\prime \prime}+\delta_{k}^{\prime} .
$$

In particular this is the order of magnitude of the subdominant term. Therefore, to obtain a descending expansion in steps of at least $k^{-\epsilon_{0}}$, we obtain the following necessary and sufficient condition on $\left(\delta_{k}^{\prime}, \delta_{k}^{\prime \prime}\right)$ :

$$
\eta_{k} \leq C k^{-\epsilon_{0}} .
$$

Under this condition, the series and remainder will go down in steps of $k^{-\epsilon_{0}}$.

With these choices of $\left(\delta_{k}^{\prime}, \delta_{k}^{\prime \prime}\right)$, the complex stationary phase expansion gives an asymptotic expansion in powers of $k^{-\epsilon_{0}}$. Recalling that the unique critical point occurs at $\theta^{\prime \prime}=0$, the remaining $d \theta^{\prime}$ integral is given by the dimensional constant $C_{m}(2 \pi)^{-r}$ times

$$
\mathscr{P}_{h^{k}}(\alpha) \sim\left(\operatorname{det}\left(k^{-1} G_{\varphi}^{\prime \prime}\left(\left|z^{\prime \prime}\right|^{2}\right)\right)^{1 / 2} \int_{\mathbf{T}^{r}} e^{i k\left\langle\alpha^{\prime} / k, \theta^{\prime}\right\rangle} \sum_{j=1}^{M} k^{-j}\left(\left\langle G_{\varphi}^{\prime \prime}\left(\left|z^{\prime \prime}\right|^{2}\right) D_{\theta^{\prime \prime}}, D_{\theta^{\prime \prime}}\right\rangle\right)^{j} \chi_{\delta} A_{k}^{\prime \prime}\left(\theta^{\prime}, 0\right) d \theta^{\prime},\right.
$$

plus the integral of the remainder (158), which is uniform in $\theta^{\prime}$ and integrates to a remainder of the same order. Here we wrote the amplitude as $A_{k}^{\prime \prime}\left(\theta^{\prime}, \theta^{\prime \prime}\right)$ and set $\theta^{\prime \prime}=0$ after the differentiations.

The differentiations leave the factor $E_{k}$ (151) while bringing down polynomials in the derivatives of its phase. The same is true of the factor $e^{k O\left(\left\|z^{\prime}\right\|^{4}\right)}$ that we absorbed into the amplitude. We now collect these factors and note that the exponent is simply the original phase (149) evaluated at $\theta^{\prime \prime}=0$ :

$$
\Phi^{\prime}\left(\theta^{\prime} ;\left|z^{\prime}\right|^{2},\left|z^{\prime \prime}\right|^{2}\right):=F_{\mathbb{C}}\left(e^{i \theta^{\prime}}\left|z^{\prime}\right|^{2},\left|z^{\prime \prime}\right|^{2}\right)-F\left(\left|z^{\prime}\right|^{2},\left|z^{\prime \prime}\right|^{2}\right)-i\left\langle\frac{\alpha^{\prime}}{k}, \theta^{\prime}\right\rangle .
$$

We also collect the derivatives of this phase and the other factors of $A_{k}$ and find that

$$
\sum_{j=1}^{M} k^{-j}\left(\left\langle G_{\varphi}^{\prime \prime}\left(\left|z^{\prime \prime}\right|^{2}\right) D_{\theta^{\prime \prime}}, D_{\theta^{\prime \prime}}\right\rangle\right)^{j} \chi_{\delta} A_{k}^{\prime \prime}\left(\theta^{\prime}, 0\right)=e^{k \Phi^{\prime}\left(\theta^{\prime} ;\left|z^{\prime}\right|^{2},\left|z^{\prime \prime}\right|^{2}\right)} \tilde{A}_{k}\left(\theta^{\prime}\right)
$$

where $\tilde{A}_{k}\left(\theta^{\prime}\right)$ is a classical symbol in $k$ whose order is the order $m$ of the original symbol $A_{k}$. The integral (160) then takes the form

$$
\mathscr{P}_{h^{k}}(\alpha) \sim C_{m}\left(\operatorname{det}\left(k^{-1} G_{\varphi}^{\prime \prime}\left(\left|z^{\prime \prime}\right|^{2}\right)\right)\right)^{1 / 2} \int_{\mathbf{T}^{r}} e^{k \Phi^{\prime}\left(\theta^{\prime} ;\left|z^{\prime}\right|^{2},\left|z^{\prime \prime}\right|^{2}\right)} \tilde{A}_{k}\left(\theta^{\prime}\right) d \theta^{\prime}
$$

This is a corner type integral as studied in Section $6 \mathrm{C}$, with $\left|z^{\prime \prime}\right|^{2}$ as an additional parameter. The asymptotics of (163) are given by Lemma 6.4. It is only necessary to keep track of the powers of $\left|z^{\prime}\right|^{2},\left|z^{\prime \prime}\right|^{2}$ and of the parameter $k^{-1}\left(\delta_{k}^{\prime \prime}\right)^{-1}\left(k \delta_{k}^{\prime}\right)^{2}$ in the analysis of $\tilde{A}_{k}$. 
To do so, we first observe that

$$
\nabla_{\theta^{\prime}} F_{\mathbb{C}}\left(e^{i \theta^{\prime}}\left|z^{\prime}\right|^{2},\left|z^{\prime \prime}\right|^{2}\right)=i \mu_{\mathbb{C}}^{\prime}\left(\left(z^{\prime}, z^{\prime \prime}\right),\left(e^{i \theta^{\prime}} z^{\prime}, z^{\prime \prime}\right)\right),
$$

that is, it is the ' component of the complexified moment map. By definition of $\left(z^{\prime}, z^{\prime \prime}\right)$ it equals $\alpha^{\prime} / k$ when $\theta^{\prime}=0$. It follows that $F_{1}^{\prime}\left(0,\left|z^{\prime \prime}\right|^{2}\right)\left|z^{\prime}\right|^{2}=\alpha^{\prime} / k$, and the almost analytic extension satisfies

$$
F_{1}^{\prime}\left(0,\left|z^{\prime \prime}\right|^{2}\right) e^{i \theta^{\prime}}\left|z^{\prime}\right|^{2}=e^{i \theta^{\prime}} \frac{\alpha^{\prime}}{k}
$$

where (as previously) the multiplication is componentwise. If we then Taylor expand the phase, we obtain

$$
\Phi^{\prime}\left(\theta^{\prime} ;\left|z^{\prime}\right|^{2},\left|z^{\prime \prime}\right|^{2}\right)=F_{1}^{\prime}\left(0,\left|z^{\prime \prime}\right|^{2}\right)\left|z^{\prime}\right|^{2}\left(1-e^{i \theta^{\prime}}\right)+O\left(\left|z^{\prime}\right|^{4}\right)=\frac{\alpha^{\prime}}{k}\left(1-e^{i \theta^{\prime}}\right)+O\left(\left|z^{\prime}\right|^{4}\right) .
$$

If we absorb the $e^{k O\left(|z|^{4}\right)}$ factor into the amplitude, the integral has now been converted to the form (146) with a more complicated amplitude.

We next observe that

$$
\tilde{A}_{k}=k^{m}\left(1+O\left(\left|z^{\prime}\right|^{2}\right)\right) \text {. }
$$

Hence, the assumption $\left|z^{\prime}\right|^{2}=O\left(\delta_{k}^{\prime}\right)$ implies that to leading order

$$
\begin{aligned}
\mathscr{P}_{h^{k}}(\alpha) & \sim \sqrt{\operatorname{det} k^{-1} G_{\varphi}^{\prime \prime}\left(\left|z^{\prime \prime}\right|^{2}\right)} k^{m} \int_{T^{r}} e^{-k\left(\left(e^{i \theta^{\prime}}-1-i \theta\right)\right) \alpha^{\prime} / k} d \theta^{\prime}\left(1+O\left(\delta_{k}^{\prime}\right)\right) \\
& =k^{m-p / 2} \sqrt{\operatorname{det} G_{\varphi}^{\prime \prime}\left(\left|z^{\prime \prime}\right|^{2}\right)} \mathscr{P}_{h_{B F}^{k}}\left(\alpha^{\prime}\right)\left(1+O\left(\delta_{k}^{\prime}\right)\right) .
\end{aligned}
$$

This completes the proof of the lemma.

\section{E. Completion of proof of Proposition 6.1.}

6E1. Asymptotic expansion for $\mathscr{P}_{h^{k}}(\alpha)$. The error terms for the asymptotics of $\mathscr{P}_{h^{k}}(\alpha)$ are $k^{-1}\left(\delta_{k}^{\prime \prime}\right)^{-1}$ in the corner zone, $k\left(\delta_{k}^{\prime}\right)^{2}$ in the interior zone and $\eta_{k}=k^{-1}\left(\delta_{k}^{\prime \prime}\right)^{-1}+k\left(\delta_{k}^{\prime}\right)^{2}+k\left(\delta_{k}^{\prime}\right)^{2} \delta_{k}^{\prime \prime}+\delta_{k}^{\prime}$ in the mixed zone. To minimize these terms, we let

$$
k^{-1}\left(\delta_{k}^{\prime \prime}\right)^{-1}=k\left(\delta_{k}^{\prime}\right)^{2} \quad \text { and } \quad 0<\delta_{k}^{\prime} \leq \delta_{k}^{\prime \prime} .
$$

By elementary calculation, the optimal choice for $\delta_{k}^{\prime}$ and $\delta_{k}^{\prime \prime}$ is given by

$$
\delta_{k}^{\prime}=\delta_{k}^{\prime \prime}=k^{-2 / 3} \quad \text { and } \quad k^{-1}\left(\delta_{k}^{\prime \prime}\right)^{-1}=k\left(\delta_{k}^{\prime}\right)^{2}
$$

and

$$
k^{-1}\left(\delta_{k}^{\prime \prime}\right)^{-1}=k\left(\delta_{k}^{\prime}\right)^{2}=k^{-1 / 3}, \quad \eta_{k} \sim O\left(k^{-1 / 3}\right) .
$$

We let $\delta_{k}=k^{-2 / 3}$ and break up the estimate into four cases.

(1) $\left|x^{\prime}\right|,\left|x^{\prime \prime}\right| \leq \delta_{k}$ : this is the corner case handled in Lemma 6.4 if $k\left(\delta_{k}\right)^{2} \rightarrow 0$.

$$
\mathscr{P}_{h^{k}}(\alpha)=C_{m} \mathscr{P}_{h_{B F}^{k}}(\alpha)\left(1+O\left(k^{-1 / 3}\right)\right) .
$$


(2) $\left|x^{\prime}\right|,\left|x^{\prime \prime}\right| \geq \delta_{k}$. By Lemma 6.3 , the stationary phase is valid and

$$
\mathscr{P}_{h^{k}}(\alpha) \sim C_{m} k^{m / 2} \sqrt{\operatorname{det} G_{\varphi}\left(\frac{\alpha}{k}\right)}\left(1+O\left(k^{-1 / 3}\right)\right) .
$$

(3) $\left|x^{\prime}\right| \leq \delta_{k}$ and $\left|x^{\prime \prime}\right| \geq \delta_{k}$. By Lemma 6.5,

$$
\mathscr{P}_{h^{k}}(\alpha)=C_{m} k^{m-(p / 2)} \sqrt{\operatorname{det} G_{\varphi}^{\prime \prime}\left(\frac{\alpha}{k}\right)} \mathscr{P}_{P, k, \delta_{k}^{\prime}}\left(\alpha^{\prime}\right)\left(1+O\left(k^{-1 / 3}\right)\right) .
$$

(4) $\left|x^{\prime \prime}\right| \leq \delta_{k}$ and $\left|x^{\prime}\right| \geq \delta_{k}$. This case is the same as case (3) by switching $x^{\prime}$ and $x^{\prime \prime}$.

Combining the formulas above, the asymptotics for $\mathscr{P}_{h^{k}}(\alpha)$ is given by (132)

$$
\mathscr{P}_{h^{k}}(\alpha)=C_{m} k^{1 / 2\left(m-\delta_{k}^{\#}(\alpha / k)\right)} \sqrt{\mathscr{G}_{\varphi, \delta_{k}}\left(\frac{\alpha}{k}\right)} \mathscr{P}_{P, k, \delta_{k}}\left(\frac{\alpha}{k}\right)\left(1+R_{k}\left(\frac{\alpha}{k}, h\right)\right),
$$

where $R_{k}(\alpha / k, h)=O\left(k^{-1 / 3}\right)$.

On the other hand, (131) is derived by the calculation

$$
\begin{aligned}
k^{1 / 2\left(m-\delta_{k}^{*}\right)} \sqrt{\mathscr{G}_{\varphi, \delta_{k}}\left(\frac{\alpha}{k}\right)} \mathscr{P}_{P, k, \delta_{k}}\left(\frac{\alpha}{k}\right) & \\
= & k^{(m / 2)} \sqrt{\operatorname{det} G_{\varphi}\left(\frac{\alpha}{k}\right)} \tilde{\mathscr{P}}_{P, k} \prod_{j \notin \mathscr{F}_{\delta_{k}}(x)}\left(2 \pi k \ell_{j}\left(\frac{\alpha}{k}\right)\right)^{-1 / 2} e^{\left|k \ell_{j}(\alpha / k)\right|} \frac{k \ell_{j}\left(\frac{\alpha}{k}\right)}{k \ell_{j}\left(\frac{\alpha}{k}\right)^{k \ell_{j}(\alpha / k)}} \\
= & k^{m / 2} \sqrt{\operatorname{det} G_{\varphi}\left(\frac{\alpha}{k}\right)} \tilde{\mathscr{P}}_{P, k}\left(1+O\left(k^{-1 / 3}\right)\right),
\end{aligned}
$$

where the last equality follows from the Stirling approximation.

6E2. Derivatives with respect to metric parameters. Now suppose that $h=h_{t}$ is a smooth one-parameter family of metrics. We would like to obtain asymptotics $(\partial / \partial t)^{j} \mathscr{P}_{h_{t}^{k}}(\alpha)$ for $j=1,2$.

Proposition 6.6. For $j=1,2$, there exist amplitudes $S_{j}$ of order zero such that

$$
\left(\frac{\partial}{\partial t}\right)^{j} \mathscr{P}_{h_{t}^{k}}(\alpha)=C_{m} k^{\left(m-\delta_{k}^{\#}(\alpha / k)\right) / 2} \sqrt{\mathscr{G}_{\varphi_{t}, \delta_{k}}\left(\frac{\alpha}{k}\right)} \mathscr{P}_{P, k, \delta_{k}}\left(\frac{\alpha}{k}\right)\left(S_{j}(t, \alpha, k)+R_{k}\left(\frac{\alpha}{k}, h_{t}\right)\right),
$$

where $R_{k}=O\left(k^{-1 / 3}\right)$. The expansion is uniform in $h$ and may be differentiated in $h$ twice with a remainder of the same order.

Proof. Such time derivatives may also be represented in the form (114)

$$
\left(\frac{\partial}{\partial t}\right)^{j} \mathscr{P}_{h_{t}^{k}}(\alpha)=(2 \pi)^{-m} \int_{\mathbf{T}^{m}} e^{-k\left(F_{t}\left(e^{i \theta} \mu_{h_{t}}^{-1}(\alpha / k)\right)-F_{t}\left(\mu_{h_{t}}^{-1}(\alpha / k)\right)\right)} A_{k, j}(k, t, \alpha, \theta) e^{i\langle\alpha, \theta\rangle} d \theta,
$$

with a new amplitude $A_{k, j}$ that is obtained by a combination of differentiations of the original amplitude in $t$ and of multiplications by $t$ derivatives of the phase. It is easy to see that $t$ derivatives of the amplitude do not change the estimates above since they do not change the order in growth in $k$ of the amplitude. However, $t$ derivatives of the phase bring down factors $k(\partial / \partial t)^{j}\left(F_{t}\left(e^{i \theta} \mu_{h_{t}}^{-1}(\alpha / k)\right)-F_{t}\left(\mu_{h_{t}}^{-1}(\alpha / k)\right)\right.$. The 
second derivative can bring down two factors with $j=1$ or one factor with $j=2$. We now verify that, despite the extra factor of $k$, the new oscillatory integral still satisfies the same estimates as before.

The key point is that, by the calculation (118), the phase $F_{t}\left(e^{i \theta} \mu_{h_{t}}^{-1}(\alpha / k)\right)-F_{t}\left(\mu_{h_{t}}^{-1}(\alpha / k)\right)-i\langle\alpha / k, \theta\rangle$ for any metric $h$ vanishes to order 2 at the critical point $\theta=0$; the first derivative vanishes because $\left.\nabla_{\theta} F_{t}\left(e^{i \theta} z\right)\right|_{\theta=0}=i \mu_{h_{t}}(z)$. Hence, the $t$ derivative of the $h_{t}$-dependent Taylor expansion (118) for a one-parameter family $h_{t}$ of metrics also vanishes to order 2 , that is,

$$
\left(\frac{\partial}{\partial t}\right)^{j}\left(F_{t}\left(e^{i \theta} \mu_{h_{t}}^{-1}\left(\frac{\alpha}{k}\right)\right)-F_{t}\left(\mu_{h_{t}}^{-1}\left(\frac{\alpha}{k}\right)\right)\right)=O\left(|\theta|^{2}\right) .
$$

Thus, for each new power of $k$ one obtains by differentiating the phase factor in $t$ one obtains a factor which vanishes to order 2 at $\theta=0$. As a check, we note that in the Bargmann-Fock model, the phase has the form $\sum_{j}\left(e^{i \theta_{j}}-1-i \theta_{j}\right) \alpha_{j} / k$.

We start with the first derivative, repeating the asymptotic analysis but with the new amplitude $S_{1}$. In the "interior region" the stationary phase calculation in Lemma 6.2 proceeds as before, but the leading term (now of one higher order than before) vanishes since it contains the value of (170) at the critical point as a factor. Therefore the asymptotics start at the same order as before but with the value of the second $\theta$-derivative of the amplitude at $\theta=0$.

In the corner and mixed boundary zones we obtain an integral of the same type as the ones studied in Lemma 6.4 and Lemma 6.5, respectively, but again with an amplitude of one higher order given by the $t$-derivative of the phase. The only change in the calculation is in the Taylor expansion of the amplitude in (167) in the $z^{\prime}$ variable, which now has the form

$$
\tilde{A}_{k, 1}=k\left(\frac{\partial}{\partial t}\right)\left(F_{t}\left(e^{i \theta} \mu_{h_{t}}^{-1}\left(\frac{\alpha}{k}\right)\right)-F_{t}\left(\mu_{h_{t}}^{-1}\left(\frac{\alpha}{k}\right)\right)\right)+O\left(\left|z^{\prime}\right|^{2}\right),
$$

so that the final integral now has the form

$$
(2 \pi)^{-m} k^{m} \int_{T^{r}} e^{-k\left(e^{i \theta^{\prime}}-1-i \theta^{\prime}\right) \alpha^{\prime} / k}\left(k\left(\frac{\partial}{\partial t}\right)\left(F_{t}\left(e^{i \theta} \mu_{h_{t}}^{-1}\left(\frac{\alpha}{k}\right)\right)-F_{t}\left(\mu_{h_{t}}^{-1}\left(\frac{\alpha}{k}\right)\right)\right)\right)_{\theta^{\prime \prime}=0} d \theta^{\prime} .
$$

As noted in (170)

$$
\begin{aligned}
k\left(\frac{\partial}{\partial t}\right)\left(F_{t}\left(e^{i \theta} \mu_{h_{t}}^{-1}\left(\frac{\alpha}{k}\right)\right)-F_{t}\left(\mu_{h_{t}}^{-1}\left(\frac{\alpha}{k}\right)\right)\right) & =k\left(\frac{\partial}{\partial t}\right)\left(F_{t}\left(e^{i \theta} \mu_{h_{t}}^{-1}\left(\frac{\alpha}{k}\right)\right)-F_{t}\left(\mu_{h_{t}}^{-1}\left(\frac{\alpha}{k}\right)\right)-i\left\langle\frac{\alpha}{k}, \theta\right\rangle\right) \\
& =k \frac{\partial}{\partial t} \int_{0}^{1}(1-s) \frac{\partial^{2}}{\partial s^{2}}\left(F_{t}\left(e^{i s \theta} \mu_{h_{t}}^{-1}\left(\frac{\alpha}{k}\right)\right)\right) d s \\
& =O\left(k|\theta|^{2} \frac{\alpha}{k}\right) .
\end{aligned}
$$

Since the stationary phase method applies as long as $|\alpha| \rightarrow \infty$ we may assume that $|\alpha| \leq C$ and we see that the factor is then bounded. Here, we have suppressed the subscript $\mathbb{C}$ for the almost-analytic extension to simplify the writing.

As an independent check, we use integration by parts in $\theta^{\prime}$. We use a cutoff function $\chi$ supported near $\theta^{\prime}=0$ to decompose the integral into a term supported near $\theta^{\prime}=0$ and one supported away from $\theta^{\prime}=0$. 
We use the integration by parts operator

$$
\mathscr{L}=\frac{1}{\left(\left(e^{i \theta^{\prime}}-1\right) \alpha^{\prime}\right)^{2}}\left(e^{i \theta^{\prime}}-1\right) \alpha^{\prime} \cdot \nabla_{\theta},
$$

where we note that the factors of $k$ cancel. The operator is well defined for $\theta^{\prime} \neq 0$ and repeated partial integration gives decay in $\alpha^{\prime}$ in case $\left|\alpha^{\prime}\right| \rightarrow \infty$. On the support of $\chi$ the denominator is not well defined but the vanishing of the phase to order two shows that $\mathscr{L}^{t}\left(S_{1}\right)$ is bounded.

Now we consider second time derivatives. The second $\partial / \partial t$ could be applied to the phase factor $e^{k \Phi_{t}}$ again or it could be applied again to (171), and then we have

$$
\begin{aligned}
\tilde{A}_{k, 2}=k\left(\frac{\partial}{\partial t}\right)\left(F_{t}\left(e^{i \theta} \mu_{h_{t}}^{-1}\left(\frac{\alpha}{k}\right)\right)-\right. & \left.F_{t}\left(\mu_{h_{t}}^{-1}\left(\frac{\alpha}{k}\right)\right)\right)^{2} \\
& +k\left(\frac{\partial^{2}}{\partial t^{2}}\right)\left(F_{t}\left(e^{i \theta} \mu_{h_{t}}^{-1}\left(\frac{\alpha}{k}\right)\right)-F_{t}\left(\mu_{h_{t}}^{-1}\left(\frac{\alpha}{k}\right)\right)\right)^{2}+O\left(\left|z^{\prime}\right|^{2}\right) .
\end{aligned}
$$

The first term contains the factor $k^{2}$ and after cancellation it induces a term of order $\left|\alpha^{\prime}\right|^{2}$. In addition this term vanishes to order four at $\theta=0$. Hence the stationary phase calculation in the case of the first derivative equally shows that the first two terms vanish and thus the factors of $k^{2}$ are canceled. In the regime where stationary phase is not applicable, $\left|\alpha^{\prime}\right|^{2}$ may be assumed bounded, and additionally one can integrate by parts twice. Thus again this term is bounded.

6F. Completion of the proof of Lemma 1.3. So far we have only considered the asymptotics of $\mathscr{P}_{h^{k}}(t, z)$. We now take the ratios to complete the proof of Lemma 1.3.

Lemma 6.7. With $\delta_{\varphi}$ defined by (58), we have

$$
\mathscr{R}_{k}(t, \alpha)=\left(\frac{\operatorname{det} \nabla^{2} u_{t}\left(\frac{\alpha}{k}\right)}{\left(\operatorname{det} \nabla^{2} u_{0}\left(\frac{\alpha}{k}\right)\right)^{1-t}\left(\operatorname{det} \nabla^{2} u_{1}\left(\frac{\alpha}{k}\right)\right)^{t}}\right)^{1 / 2}\left(1+O\left(k^{-1 / 3}\right)\right) .
$$

The asymptotic may be differentiated twice with the same order of remainder.

Proof. Combining Corollary 3.2 and Proposition 6.1, we have

$$
\mathscr{R}_{k}(t, \alpha)=\frac{\sqrt{\operatorname{det} G_{\varphi_{t}}\left(\frac{\alpha}{k}\right)} \tilde{\mathscr{P}}_{P, k}\left(\frac{\alpha}{k}\right)}{\left(\sqrt{\operatorname{det} G_{\varphi_{0}}\left(\frac{\alpha}{k}\right)} \tilde{\mathscr{P}}_{P, k}\left(\frac{\alpha}{k}\right)\right)^{1-t}\left(\sqrt{\operatorname{det} G_{\varphi_{1}}\left(\frac{\alpha}{k}\right)} \tilde{\mathscr{P}}_{P, k}\left(\frac{\alpha}{k}\right)\right)^{t}}\left(1+O\left(k^{-1 / 3}\right)\right) .
$$

We observe that the factors of $\tilde{\mathscr{P}}_{P, k}$ cancel out, leaving

$$
\mathscr{R}_{k}(t, \alpha)=\frac{\sqrt{\operatorname{det} G_{\varphi_{t}}\left(\frac{\alpha}{k}\right)}}{\left(\sqrt{\operatorname{det} G_{\varphi_{0}}\left(\frac{\alpha}{k}\right)}\right)^{1-t}\left(\sqrt{\operatorname{det} G_{\varphi_{1}}\left(\frac{\alpha}{k}\right)}\right)^{t}}\left(1+O\left(k^{-1 / 3}\right)\right) .
$$

By Proposition 6.6, the asymptotic in (173) may be differentiated twice with the same order of remainder, completing the proof. 
Remark. By (58), we also have

$$
\mathscr{R}_{k}(t, \alpha)=\left(\frac{\delta_{\varphi_{0}}^{1-t} \delta_{\varphi_{1}}^{t}}{\delta_{\varphi_{t}}}\right)^{-1 / 2}\left(1+O\left(k^{-1 / 3}\right)\right) .
$$

Indeed, the factors of $\ell_{j}(\alpha / k)$ are independent of the metrics and cancel out. Also $\left(\delta_{\varphi_{0}}^{1-t} \delta_{\varphi_{1}}^{t} / \delta_{\varphi_{t}}\right)^{-1 / 2}$ is smooth on $P$.

The following simpler estimate on logarithmic derivatives is sufficient for much of the proof of the main results:

Lemma 6.8. Both $\partial_{t} \log \mathscr{R}_{k}(t, \alpha)$ and $\partial_{t}^{2} \log \mathscr{R}_{k}(t, \alpha)$ are uniformly bounded.

Proof. We first note that

$$
\partial_{t} \log \mathscr{R}_{k}(t, \alpha)=\log \mathscr{P}_{h_{1}^{k}}(\alpha)-\log \mathscr{P}_{h_{0}^{k}}(\alpha)-\partial_{t} \log \mathscr{P}_{h_{t}^{k}}(\alpha)
$$

By Proposition 6.1,

$$
\log \mathscr{P}_{h^{k}}(\alpha)=\frac{1}{2} \log \operatorname{det}\left(k^{-1} G_{\varphi}\left(\frac{\alpha}{k}\right)\right)+\log \tilde{\mathscr{P}}_{P, k}\left(\frac{\alpha}{k}\right)+\log C_{m}+O\left(k^{-1 / 3}\right) .
$$

As in Lemma 6.7, the Bargmann-Fock terms cancel between the $h_{0}$ and $h_{1}$ terms, while the metric factors simplify asymptotically to $\frac{1}{2} \log \left(\delta_{\varphi_{1}} \delta_{\varphi_{0}}\right)$, and this is clearly bounded. To complete the proof that $\partial_{t} \log \mathscr{R}_{k}(t, \alpha)$ is uniformly bounded, we need the final ratio to be bounded. By Proposition 6.6, we see that in the "interior" region both numerator and denominator have asymptotics which differ only in the value of a zeroth order amplitude at $\theta=0$ and that it equals 1 in the case of the denominator. Hence, the ratio is bounded in the interior. Towards the boundary, the denominator is comparable with the Bargmann-Fock model and is bounded below by one. The numerator is also bounded by Proposition 6.6, and therefore the ratio is everywhere bounded.

Now we consider the case of $\partial_{t}^{2} \log \mathscr{R}_{k}(t, \alpha)$, which simplifies to

$$
\partial_{t}^{2} \log \mathscr{R}_{k}(t, \alpha)=-\frac{\partial_{t}^{2} \mathscr{P}_{h_{t}^{k}}(\alpha)}{\mathscr{P}_{h_{t}^{k}}(\alpha)}+\left(\frac{\partial_{t} \mathscr{P}_{h_{t}^{k}}(\alpha)}{\mathscr{P}_{h_{t}^{k}}(\alpha)}\right)^{2} .
$$

As we have just argued, the second factor is bounded. The same argument applies to the first term by Proposition 6.6.

\section{7. $C^{0}$ and $C^{1}$-convergence}

We begin with the rather simple proof of $C^{0}$-convergence with remainder bounds.

7A. Co-convergence.

Proposition 7.1. $\frac{1}{k} \log Z_{k}(t, z) e^{-k \varphi_{t}(z)}=O\left(\frac{\log k}{k}\right)$ uniformly for $(t, z) \in[0,1] \times M$.

We will derive the proposition from the following result, which in turn is an immediate consequence of Lemma 6.7:

Lemma 7.2 (upper/lower bound lemma). There exist $C, c>0$ such that

$$
c \leq \mathscr{R}_{k}(t, \alpha) \leq C .
$$


Proof of Proposition 7.1. By the upper/lower bound lemma, there exist constants $c, C>0$ such that

$$
c \Pi_{h_{t}^{k}}(z, z) \leq \sum_{\alpha \in k P \cap \mathbb{Z}^{m}} \mathscr{R}_{k}(t, \alpha) \frac{\left|S_{\alpha}(z)\right|_{h_{t}^{k}}^{2}}{2_{h_{t}^{k}}(\alpha)} \leq C \Pi_{h_{t}^{k}}(z, z) .
$$

Hence,

$$
\frac{1}{k} \log \Pi_{h_{t}^{k}}(z, z) \leq \frac{1}{k} \log \sum_{\alpha \in k P \cap \mathbb{Z}^{m}} \mathscr{T}_{k}(t, \alpha) \frac{\left|S_{\alpha}(z)\right|_{h_{t}^{k}}^{2}}{2_{h_{t}^{k}}(\alpha)} \leq \frac{1}{k} \log \Pi_{h_{t}^{k}}(z, z)+O\left(\frac{1}{k}\right)=O\left(\frac{\log k}{k}\right),
$$

where the last estimate follows from (93).

7B. $C^{\mathbf{1}}$-convergence. We now discuss first derivatives in $(t, z)$. In the $z$ variable the vector fields $\partial / \partial \rho_{j}$ vanish on $\mathscr{D}$, so we can only use them to estimate $C^{1}$ norms in directions $\delta_{k}$ far from the boundary. In directions close to the boundary we may choose coordinates so that derivatives in $z^{\prime}$ near $z^{\prime}=0$ define the $C^{1}$ norm.

The estimates in the $\rho$ and $z^{\prime}$ derivatives are similar. We carry out the calculations in detail in the $\rho$ variables and then indicate how to carry out the analogous estimates in the $z$ variable.

We also consider the $t$ derivative. The key distinction between $t$ and $z$ derivatives is the following:

- $z$ or $\rho$ derivatives bring down derivatives of the phase, which have the form $k\left(\mu_{h_{t}}(z)-\alpha / k\right)$. The factor of $k$ raises the order of asymptotics while the factor $\left(\mu_{h_{t}}(z)-\alpha / k\right)$ lowers it by the Localization Lemma.

- $t$ derivatives do not apply to the phase and only differentiate $\mathscr{R}_{k}(t, \alpha)$ and $\mathscr{2}_{h_{t}^{k}}(\alpha)$.

Proposition 7.3. Uniformly for $(t, z) \in[0,1] \times M$, we have:

(1) $\frac{1}{k}\left|\frac{\partial}{\partial \rho_{i}} \log \sum_{\alpha \in k P \cap \mathbb{Z}^{m}} \mathscr{R}_{k}(t, \alpha) \frac{\left|S_{\alpha}(z)\right|_{h_{t}^{k}}^{2}}{2_{h_{t}^{k}}(\alpha)}\right|=O\left(k^{-1 / 2+\delta}\right)$.

(2) The same estimate is valid for the derivative $\partial / \partial r_{n}$ in directions near $\mathscr{D}$, as in Proposition 4.6.

(3) $\frac{1}{k}\left|\frac{\partial}{\partial t} \log \sum_{\alpha \in k P \cap \mathbb{Z}^{m}} \mathscr{R}_{k}(t, \alpha) \frac{\left|S_{\alpha}(z)\right|_{h_{t}^{k}}^{2}}{2_{h_{t}^{k}}(\alpha)}\right|=O\left(k^{-1 / 3}\right)$.

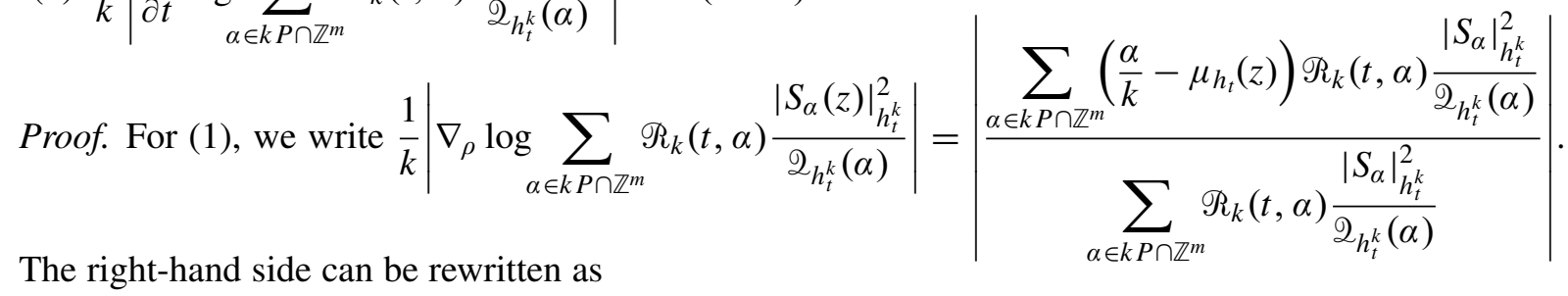

$$
\left|\frac{\sum_{\substack{\alpha \in k P \cap \mathbb{Z}^{m} \\\left|\alpha / k-\mu_{h_{t}}(z)\right| \leq k^{-1 / 2+\delta}}}\left(\frac{\alpha}{k}-\mu_{h_{t}}(z)\right) \mathscr{R}_{k}(t, \alpha) \frac{\left|S_{\alpha}\right|_{h_{t}^{k}}^{2}}{\mathscr{Q}_{h_{t}^{k}}(\alpha)}}{\sum_{\alpha \in k P \cap \mathbb{Z}^{m}} \mathscr{R}_{k}(t, \alpha) \frac{\left|S_{\alpha}\right|_{h_{t}^{k}}^{2}}{\mathscr{2}_{h_{t}^{k}}(\alpha)}}\right|+O\left(k^{-M}\right),
$$


which in turn is bounded above by

$$
C k^{-1 / 2+\delta}\left|\frac{\sum_{\substack{\alpha \in k P \cap \mathbb{Z}^{m} \\\left|\alpha / k-\mu_{h_{t}}(z)\right| \leq k^{-1 / 2+\delta}}}\left|S_{\alpha}\right|_{h_{t}^{k}}^{2} / \mathscr{Q}_{h_{t}^{k}}(\alpha)}{\sum_{\alpha \in k P \cap \mathbb{Z}^{m}}\left|S_{\alpha}\right|_{h_{t}^{k}}^{2} / \mathscr{2}_{h_{t}^{k}}(\alpha)}\right|+O\left(k^{-M}\right) \leq C k^{-1 / 2+\delta},
$$

proving (1). Here we have applied the Localization Lemma 1.2 and Lemma 7.2 to $\mathscr{R}_{k}$.

Regarding the derivatives $\partial / \partial r_{n}$ in (2), the only change to the argument is in summing only $\alpha$ with $\alpha_{n} \neq 0$ and then changing $\alpha$ to $\alpha-\left(0, \ldots, 1_{n}, \ldots, 0\right)$ as explained in Proposition 4.6. Clearly the localization and the estimates only change by $1 / k$.

We now consider the $\partial_{t}$ derivative. By Proposition 4.4, we have

$\frac{1}{k} \frac{\partial}{\partial t} \log \sum_{\alpha \in k P \cap \mathbb{Z}^{m}} \mathscr{R}_{k}(t, \alpha) \frac{\left|S_{\alpha}(z)\right|_{h_{t}^{k}}^{2}}{2_{h_{t}^{k}}(\alpha)}$

$$
\begin{aligned}
& =\frac{1}{k} \frac{\sum_{\alpha} \mathscr{R}_{k}(t, \alpha) \partial_{t} \log \left(\frac{\mathscr{R}_{k}(t, \alpha)}{\mathscr{2}_{h_{t}^{k}}(\alpha)}\right) \frac{e^{\langle\alpha, \rho\rangle}}{2_{t}^{k}(\alpha)}}{\sum_{\alpha} \mathscr{R}_{k}(t, \alpha) \frac{e^{\langle\alpha, \rho\rangle}}{\mathscr{2}_{h_{t}^{k}}(\alpha)}}-\frac{\partial}{\partial t} \varphi_{t} \\
& =\frac{1}{k} \frac{\sum_{\alpha} \mathscr{R}_{k}(t, \alpha) \partial_{t} \log \left(\frac{\mathscr{R}_{k}(t, \alpha)}{\mathscr{2}_{h_{t}^{k}}(\alpha)}\right) \frac{e^{\langle\alpha, \rho\rangle}}{\mathscr{2}_{h_{t}^{k}}(\alpha)}}{\sum_{\alpha} \mathscr{R}_{k}(t, \alpha) \frac{e^{\langle\alpha, \rho\rangle}}{\mathscr{2}_{h_{t}^{k}}(\alpha)}}-\frac{1}{k} \frac{\sum_{\alpha} \partial_{t} \log \left(\frac{1}{\mathscr{Q}_{h_{t}^{k}}(\alpha)}\right) \frac{e^{\langle\alpha, \rho\rangle}}{\mathscr{2}_{h_{t}^{k}}(\alpha)}}{\sum_{\alpha} \frac{e^{\langle\alpha, \rho\rangle}}{\mathscr{2}_{h_{t}^{k}}(\alpha)}}+O\left(k^{-1}\right) \\
& =\frac{1}{k} \frac{\sum_{\alpha} \mathscr{R}_{k}(t, \alpha) \partial_{t} \log \mathscr{R}_{k}(t, \alpha) \frac{e^{\langle\alpha, \rho\rangle}}{\mathscr{2}_{h_{t}^{k}}(\alpha)}}{\sum_{\alpha} \mathscr{R}_{k}(t, \alpha) \frac{e^{\langle\alpha, \rho\rangle}}{2_{h_{t}^{k}}(\alpha)}} \\
& +\frac{1}{k}\left(\frac{\sum_{\alpha} \partial_{t} \log \mathscr{2}_{h_{t}^{k}}(\alpha) \frac{e^{\langle\alpha, \rho\rangle}}{2_{h_{t}^{k}}(\alpha)}}{\sum_{\alpha} \frac{e^{\langle\alpha, \rho\rangle}}{2_{h_{t}^{k}}(\alpha)}}-\frac{\sum_{\alpha} \mathscr{R}_{k}(t, \alpha) \partial_{t} \log \mathscr{2}_{h_{t}^{k}}(\alpha) \frac{e^{\langle\alpha, \rho\rangle}}{\mathscr{Q}_{h_{t}^{k}}(\alpha)}}{\sum_{\alpha} \mathscr{R}_{k}(t, \alpha) \frac{e^{\langle\alpha, \rho\rangle}}{\mathscr{2}_{h_{t}^{k}}(\alpha)}}\right)+O\left(k^{-1}\right) .
\end{aligned}
$$

Notice that $\mathscr{2}_{h_{t}^{k}}=\mathscr{R}_{k}(t, \alpha)\left(\mathscr{2}_{h_{0}^{k}}(\alpha)\right)^{1-t}\left(\mathscr{2}_{h_{1}^{k}}(\alpha)\right)^{t}$, so

$$
\partial_{t} \log \mathscr{2}_{k}(t, \alpha)=\partial_{t} \log \mathscr{R}_{k}(t, \alpha)+\log \frac{\mathscr{2}_{h_{1}^{k}}(t, \alpha)}{\mathscr{2}_{h_{0}^{k}}(t, \alpha)} .
$$

It follows easily from the fact proved in Lemma 1.3 (or more precisely the simpler Lemma 6.8) that $\mathscr{R}_{k}(t, \alpha)=O(1)$ and $\partial_{t} \log \mathscr{R}_{k}(t, \alpha)=O(1)$. Also the rightmost term in (180) is $O(k)$ uniformly in $\alpha$. Replacing $\mathscr{R}_{k}$ by $\mathscr{R}_{\infty}$ plus an error of order $k^{-1 / 3}$, we obtain, as needed,

$$
\frac{1}{k} \frac{\partial}{\partial t} \log \sum_{\alpha \in k P \cap \mathbb{Z}^{m}} \mathscr{R}_{k}(t, \alpha) \frac{\left|S_{\alpha}(z)\right|_{h_{t}^{k}}^{2}}{2_{h_{t}^{k}}(\alpha)}=O\left(k^{-1 / 3}\right) .
$$




\section{8. $C^{2}$-convergence}

We now consider second derivatives in $\rho, t$. Again we must separately consider derivatives in the interior and near the boundary. The following proposition completes the proof of Theorem 1.1.

Proposition 8.1. Uniformly for $(t, z) \in[0,1] \times M$, we have, for any $\delta>0$,

(1) $\frac{1}{k}\left|\frac{\partial^{2}}{\partial \rho_{i} \partial \rho_{j}} \log \sum_{\alpha \in k P \cap \mathbb{Z}^{m}} \mathscr{R}_{k}(t, \alpha) \frac{\left|S_{\alpha}(z)\right|_{h_{t}^{k}}^{2}}{2_{h_{t}^{k}}(\alpha)}\right|=O\left(k^{-(1 / 3)+2 \delta}\right)$;

(2) $\frac{1}{k}\left|\frac{\partial^{2}}{\partial t \partial \rho_{j}} \log \sum_{\alpha \in k P \cap \mathbb{Z}^{m}} \mathscr{R}_{k}(t, \alpha) \frac{\left|S_{\alpha}(z)\right|_{h_{t}^{k}}^{2}}{2 h_{t}^{k}(\alpha)}\right|=O\left(k^{-(1 / 3)+2 \delta}\right)$;

(3) $\frac{1}{k}\left|\frac{\partial^{2}}{\partial t^{2}} \log \sum_{\alpha \in k P \cap \mathbb{Z}^{m}} \mathscr{R}_{k}(t, \alpha) \frac{\left|S_{\alpha}(z)\right|_{h_{t}^{k}}^{2}}{2_{h_{t}^{k}}(\alpha)}\right|=O\left(k^{-(1 / 3)+2 \delta}\right)$.

(4) The same estimates are valid for the derivative $\partial / \partial r_{n}$ in directions near $\mathscr{D}$ as in Proposition 4.6.

We break up the proof into the four cases. To simplify the exposition, we introduce some new notation for localizing sums over lattice points. By the Localization Lemma 1.2, sums over lattice points can be localized to a ball of radius $O\left(k^{-1 / 2+\delta}\right)$ around $\mu_{h_{t}}(z)$. We emphasize that although there are three metrics at play, it is the metric $h_{t}$ along the Monge-Ampère geodesic that is used to localize the sum. We introduce a notation for localized sums over pairs of lattice points: let

$$
\sum_{\alpha, \beta} F(\alpha, \beta):=\sum_{\substack{\left|\alpha / k-\mu_{h_{t}}(z)\right| \leq k^{-1 / 2+\delta} \\\left|\beta / k-\mu_{h_{t}}(z)\right| \leq k^{-1 / 2+\delta}}} F(\alpha, \beta) .
$$

Notation. Throughout the calculations in Sections 6.5 and 8B, $(\alpha-\beta)$ stands for $(\alpha-\beta)_{i}$ and $(\alpha-\beta)^{2}$ stands for $(\alpha-\beta)_{i}(\alpha-\beta)_{j}$.

8A. Second space derivatives in the interior. In this section we prove case (1). We have

$$
\begin{aligned}
& \frac{1}{k}\left|\frac{\partial^{2}}{\partial \rho_{i} \partial \rho_{j}} \log \sum_{\alpha \in k P \cap \mathbb{Z}^{m}} \mathscr{R}_{k}(t, \alpha) \frac{\left|S_{\alpha}(z)\right|_{h_{t}^{k}}^{2}}{\mathscr{2}_{h_{t}^{k}}(\alpha)}\right| \\
& =\frac{1}{k}\left|\frac{\frac{1}{2} \sum_{\alpha, \beta}(\alpha-\beta)^{2} \mathscr{R}_{k}(t, \alpha) \mathscr{R}_{k}(t, \beta) \frac{e^{\langle\alpha, \rho\rangle}}{2_{h_{t}^{k}}(\alpha)} \frac{e^{\langle\beta, \rho\rangle}}{2_{h_{t}^{k}}(\beta)}}{\left(\sum_{\alpha} \mathscr{R}_{k}(t, \alpha) \frac{e^{\langle\alpha, \rho\rangle}}{2_{h_{t}^{k}}(\alpha)}\right)^{2}}-k \frac{\partial^{2}}{\partial \rho_{i} \partial \rho_{j}} \varphi_{t}\right| \\
& \equiv \frac{1}{k}\left|\frac{\frac{1}{2} \sum_{\alpha, \beta}(\alpha-\beta)^{2} \mathscr{R}_{k}(t, \alpha) \mathscr{R}_{k}(t, \beta) \frac{e^{\langle\alpha, \rho\rangle}}{2_{h_{t}^{k}}(\alpha)} \frac{e^{\langle\beta, \rho\rangle}}{2_{h_{t}^{k}}(\beta)}}{\left(\sum_{\alpha} \mathscr{R}_{k}(t, \alpha) \frac{e^{\langle\alpha, \rho\rangle}}{\mathscr{2}_{h_{t}^{k}}(\alpha)}\right)^{2}}-\frac{\frac{1}{2} \sum_{\alpha, \beta}(\alpha-\beta)^{2} \frac{e^{\langle\alpha, \rho\rangle}}{2_{h_{t}^{k}}(\alpha)} \frac{e^{\langle\beta, \rho\rangle}}{2_{h_{t}^{k}}(\beta)}}{\left(\sum_{\alpha} \frac{e^{\langle\alpha, \rho\rangle}}{2_{h_{t}^{k}}(\alpha)}\right)^{2}}\right|,
\end{aligned}
$$


modulo $O(1 / k)$ by Proposition 4.3. We also completed the square and used that the sum over $\alpha$ is a probability measure to replace $\alpha^{2}-\alpha \beta$ by $\frac{1}{2}(\alpha-\beta)^{2}$ using the symmetry in $\alpha$ and $\beta$ in the sum. We also use Proposition 4.5 to write $\partial^{2} \varphi_{t} / \partial \rho_{i} \partial \rho_{j}$ as a sum over lattice points.

By the Localization Lemma 1.2, each sum over lattice points can be localized to a ball of radius $O\left(k^{-1 / 2+\delta}\right)$ around $\mu_{h_{t}}(z)$. Then, by Lemma 1.3 each occurrence of $\mathscr{R}_{k}(t, \alpha)$ or $\mathscr{R}_{k}(t, \beta)$ may be replaced by $\mathscr{R}_{\infty}(t, \alpha / k)$ plus an error of order $k^{-1 / 3}$. Since

$$
\frac{1}{k}(\alpha-\beta)^{2}=O\left(k^{2 \delta}\right),
$$

the total error is of order $k^{2 \delta-1 / 3}$. Since $\delta$ is arbitrarily small, this term is decaying. Further, after replacing $\mathscr{R}_{k}(t, \beta)$ by $\mathscr{R}_{\infty}(t, \alpha / k)$ we may then replace $\alpha / k$ and $\beta / k$ by $\mu_{h_{t}}(z)$ at the expense of another error of order $k^{-1 / 2+\delta}$. By modifying (182) accordingly, we have

$$
\begin{aligned}
& \frac{1}{k}\left|\frac{\partial^{2}}{\partial \rho_{i} \partial \rho_{j}} \log \sum_{\alpha \in k P \cap \mathbb{Z}^{m}} \mathscr{R}_{k}(t, \alpha) \frac{\left|S_{\alpha}(z)\right|_{h_{t}^{k}}^{2}}{2_{h_{t}^{k}}(\alpha)}\right|+O\left(k^{-(1 / 3)+2 \delta}\right) \\
& \quad \equiv \frac{1}{k}\left|\frac{\frac{1}{2} \sum_{\alpha, \beta}(\alpha-\beta)^{2} \mathscr{R}_{\infty}\left(t, \mu_{h_{t}}\left(e^{\rho / 2}\right)\right)^{2} \frac{e^{\langle\alpha, \rho\rangle}}{2_{h_{t}^{k}}(\alpha)} \frac{e^{\langle\beta, \rho\rangle}}{2_{h_{t}^{k}}(\beta)}}{\left(\sum_{\alpha} \mathscr{R}_{\infty}\left(t, \mu_{h_{t}}\left(e^{\rho / 2}\right)\right) \frac{e^{\langle\alpha, \rho\rangle}}{2_{h_{t}^{k}}(\alpha)}\right)^{2}}-\frac{\frac{1}{2} \sum_{\alpha, \beta}(\alpha-\beta)^{2} \frac{e^{\langle\alpha, \rho\rangle}}{2_{h_{t}^{k}}(\alpha)} \frac{e^{\langle\beta, \rho\rangle}}{2_{h_{t}^{k}}(\beta)}}{\left(\sum_{\alpha} \frac{e^{\langle\alpha, \rho\rangle}}{2_{h_{t}^{k}}(\alpha)}\right)^{2}}\right| \equiv 0,
\end{aligned}
$$

where $\equiv$ means that the lines agree modulo errors of order $O\left(k^{-(1 / 3)+2 \delta}\right)$. In the last estimate, we use that $\mathscr{R}_{\infty}\left(t, \mu_{h_{t}}\left(e^{\rho / 2}\right)\right)^{2}$ cancels out in the first term. This completes the proof in the spatial interior case.

The modifications when $z$ is close to $\partial P$ are just as in the case of the first derivatives.

8B. Mixed space-time derivatives. The mixed space-time derivative is given by

$$
\begin{aligned}
\frac{1}{k}\left|\frac{\partial^{2}}{\partial \rho_{i} \partial t} \log \sum_{\alpha \in k P \cap \mathbb{Z}^{m}} \mathscr{R}_{k}(t, \alpha) \frac{\left|S_{\alpha}(z)\right|_{h_{t}^{k}}^{2}}{\mathscr{Q}_{h_{t}^{k}}(\alpha)}\right| \\
=\frac{1}{k}\left|\frac{1}{2} \frac{\sum_{\alpha, \beta}(\alpha-\beta) \mathscr{R}_{k}(t, \beta) \mathscr{R}_{k}(t, \alpha) \partial_{t} \log \left(\frac{\mathscr{R}_{k}(t, \alpha) \mathscr{2}_{h_{t}^{k}}(\beta)}{\mathscr{R}_{k}(t, \beta) \mathscr{2}_{h_{t}^{k}}(\alpha)}\right) \frac{e^{\langle\alpha, \rho\rangle}}{\mathscr{2}_{h_{t}^{k}}(\alpha)} \frac{e^{\langle\beta, \rho\rangle}}{\mathscr{2}_{h_{t}^{k}}(\beta)}}{\left(\sum_{\alpha} \mathscr{R}_{k}(t, \alpha) \frac{e^{\langle\alpha, \rho\rangle}}{\mathscr{2}_{h_{t}^{k}}(\alpha)}\right)^{2}}-k \frac{\partial^{2}}{\partial \rho_{i} \partial t} \varphi_{t}\right| .
\end{aligned}
$$

It suffices to prove that

$$
\frac{1}{k}\left|\frac{\sum_{\alpha, \beta}(\alpha-\beta) \partial_{t} \log \left(\mathscr{R}_{k}(t, \alpha)\right) \frac{e^{\langle\alpha, \rho\rangle}}{2_{h_{t}^{k}}(\alpha)} \frac{e^{\langle\beta, \rho\rangle}}{2_{h_{t}^{k}}(\beta)}}{\left(\sum_{\alpha} \frac{e^{\langle\alpha, \rho\rangle}}{2_{h_{t}^{k}}(\alpha)}\right)^{2}}\right|=O\left(k^{-1 / 2+\delta}\right)
$$


and

$$
\frac{1}{k}\left|\frac{1}{2} \frac{\sum_{\alpha, \beta}(\alpha-\beta) \mathscr{R}_{k}(t, \beta) \mathscr{R}_{k}(t, \alpha) \partial_{t} \log \left(\frac{2_{h_{t}^{k}}(\beta)}{2_{h_{t}^{k}}(\alpha)}\right) \frac{e^{\langle\alpha, \rho\rangle}}{2_{h_{t}^{k}}(\alpha)} \frac{e^{\langle\beta, \rho\rangle}}{2_{h_{t}^{k}}(\beta)}}{\left(\sum_{\alpha} \mathscr{R}_{k}(t, \alpha) \frac{e^{\langle\alpha, \rho\rangle}}{2_{h_{t}^{k}}(\alpha)}\right)^{2}}-k \frac{\partial^{2}}{\partial \rho_{i} \partial t} \varphi_{t}\right|=O\left(k^{-(1 / 3)+2 \delta}\right) .
$$

The first estimate follows by the Localization Lemma 1.2 and from Lemma 6.8, i.e., $\partial_{t} \log \mathscr{R}_{k}(t, \alpha)=$ $O(1)$. The second estimate is very similar to that in Section 8A, specifically in (183), so we do not write it out in full. In outline, we first apply the Localization Lemma and replace each $\mathscr{R}_{k}(t, \alpha)$ by $\mathscr{R}_{\infty}\left(\mu_{h_{t}}(z)\right)$ with $z=e^{\rho / 2}$. The errors in making these replacements are of order $k^{-1 / 3+\delta}$ because

$$
\partial_{t} \log \frac{2_{h_{t}^{k}}(\beta)}{2_{h_{t}^{k}}(\alpha)}=O\left(k\left|u_{t}(\alpha)-u_{t}(\beta)\right|\right)=O\left(k^{1 / 2+\delta}\right),
$$

and because $\alpha-\beta=O\left(k^{1 / 2+\delta}\right)$ in the localized sum. We then express $\partial^{2} \varphi_{t} / \partial \rho_{i} \partial t$ in terms of the Szegó kernel, that is, as a sum over lattice points, using Proposition 4.5, and cancel the $\partial^{2} \varphi_{t} / \partial \rho_{i} \partial t$ term. The sum of the remainders is then of order $k^{-1 / 3+\delta}$, completing the proof in this mixed case.

8C. Second time derivatives. The proof in this case follows the same pattern, although the estimates are somewhat more involved. The main steps are to localize the sums over lattice points, to replace each $\mathscr{R}_{k}$ by $\mathscr{R}_{\infty}$, then to cancel out $\mathscr{R}_{\infty}$ after all replacements, and to see that the resulting lattice point sum cancels $\partial^{2} \varphi_{t} / \partial \rho_{i} \partial t$. The complications are only due to the number of estimates that are required to justify the replacements.

The second time derivative equals

$$
\begin{aligned}
& \frac{1}{k} \frac{\partial^{2}}{\partial t^{2}} \log \sum_{\alpha \in k P \cap \mathbb{Z}^{m}} \mathscr{R}_{k}(t, \alpha) \frac{\left|S_{\alpha}(z)\right|_{h_{t}^{k}}^{2}}{2_{h_{t}^{k}}(\alpha)} \\
& =\frac{1}{k} \frac{\sum_{\alpha, \beta} \mathscr{R}_{k}(t, \beta) \mathscr{R}_{k}(t, \alpha)\left(\partial_{t} \log \left(\frac{\mathscr{R}_{k}(t, \alpha)}{\mathscr{2}_{h_{t}^{k}}(\alpha)}\left(\frac{\mathscr{R}_{k}(t, \beta)}{2_{h_{t}^{k}}(\beta)}\right)^{-1}\right)\right)^{2} \frac{e^{\langle\alpha, \rho\rangle}}{2_{h_{t}^{k}}(\alpha)} \frac{e^{\langle\beta, \rho\rangle}}{\mathscr{2}_{h_{t}^{k}}(\beta)}}{\left(\sum_{\alpha} \mathscr{R}_{k}(t, \alpha) \frac{e^{\langle\alpha, \rho\rangle}}{2_{h_{t}^{k}}(\alpha)}\right)^{2}} \\
& +\frac{1}{k}\left(\frac{\sum_{\alpha, \beta} \mathscr{R}_{k}(t, \beta) \mathscr{R}_{k}(t, \alpha) \partial_{t}^{2} \log \left(\frac{\mathscr{R}_{k}(t, \alpha)}{2_{h_{t}^{k}}(\alpha)}\right) \frac{e^{\langle\alpha, \rho\rangle}}{2_{h_{t}^{k}}(\alpha)} \frac{e^{\langle\beta, \rho\rangle}}{2_{h_{t}^{k}}(\beta)}}{\left(\sum_{\alpha} \mathscr{R}_{k}(t, \alpha) \frac{e^{\langle\alpha, \rho\rangle}}{2_{h_{t}^{k}}(\alpha)}\right)^{2}}-k \frac{\partial^{2}}{\partial t^{2}} \varphi_{t}\right) .
\end{aligned}
$$

On the middle line of (184), the square term in the numerator is a simplification of

$$
\left(\partial_{t} \log \left(\frac{\mathscr{R}_{k}(t, \alpha)}{\mathscr{2}_{h_{t}^{k}}(\alpha)}\left(\frac{\mathscr{R}_{k}(t, \beta)}{\mathscr{2}_{h_{t}^{k}}(\beta)}\right)^{-1}\right)\right)\left(\partial_{t} \log \frac{\mathscr{R}_{k}(t, \alpha)}{\mathscr{2}_{h_{t}^{k}}(\alpha)}\right),
$$


using the fact that the expression is antisymmetric in $(\alpha, \beta)$ and that we are summing over $\alpha, \beta-$ similar to what we did in (182). To simplify the notation, we introduce the abbreviations $\mathscr{R}(\alpha)=\mathscr{R}_{k}(t, \alpha), \mathscr{T}(\alpha)=\frac{1}{2_{h_{t}^{k}}(\alpha)}, f^{\prime}=\frac{\partial f}{\partial t}$,
and we write $(184)=N / D$, where the numerator has the schematic form

$N=\sum_{\alpha, \beta}\left(\left(\frac{\mathscr{R}^{\prime}}{\mathscr{R}}(\alpha)+\frac{\mathscr{T}^{\prime}}{\mathscr{T}}(\alpha)\right)^{\prime}+\frac{1}{2}\left(\frac{\mathscr{R}^{\prime}}{\mathscr{R}}(\alpha)+\frac{\mathscr{T}^{\prime}}{\mathscr{T}}(\alpha)-\left(\frac{\mathscr{R}^{\prime}}{\mathscr{R}}(\beta)+\frac{\mathscr{T}^{\prime}}{\mathscr{T}}(\beta)\right)\right)^{2}\right) \mathscr{R}(\alpha) \mathscr{T}(\alpha) \mathscr{R}(\beta) \mathscr{T}(\beta) e^{\langle\alpha, \rho\rangle} e^{\langle\beta, \rho\rangle}$,

and where the denominator is $D=\left(\sum_{\alpha} \mathscr{R}(\alpha) \mathscr{T}(\alpha)\right)^{2}$. We omit the factors $\frac{e^{\langle\alpha, \rho\rangle}}{2_{h_{t}^{k}}(\alpha)} \frac{e^{\langle\beta, \rho\rangle}}{2_{h_{t}^{k}}(\beta)}$ from the
notation since they are always present.

We now compare $N$ and $D$ to the corresponding expressions in the second time derivative of the Szegó kernel in Proposition 4.5. In the latter case, $\mathscr{R} \equiv 1$ so any terms with $t$-derivatives of $\mathscr{R}$ above do not occur in the third comparison expression of Proposition 4.5. Terms with no $t$ derivatives of $\mathscr{R}$ will be precisely as in the comparison except that $\mathscr{R}$ is replaced by 1 . So we consider a subsum of $N$ :

$$
N_{1}=\sum_{\alpha, \beta}\left(\left(\frac{\mathscr{T}^{\prime}}{\mathscr{T}}(\alpha)\right)^{\prime}+\frac{1}{2}\left(\frac{\mathscr{T}^{\prime}}{\mathscr{T}}(\alpha)-\frac{\mathscr{T}^{\prime}}{\mathscr{T}}(\beta)\right)^{2}\right) \mathscr{R}(\alpha) \mathscr{T}(\alpha) \mathscr{R}(\beta) \mathscr{T}(\beta) .
$$

If we now replace all occurrences of $\mathscr{R}_{k}(t, \alpha)$ by $\mathscr{R}_{\infty}\left(\mu_{h_{t}}(z)\right)$ in both numerator and denominator we get the Szegô kernel expression (the third comparison expression of Proposition 4.5) of order $1 / k^{2}$. (This is verified in more detail at the end of the proof.) So we are left with estimating two remainder terms: first, the difference $N_{1}-\tilde{N}_{1}$, where $\tilde{N}_{1}$ is a sum of terms in which we replace at least one $\mathscr{R}(\alpha)$ by $\mathscr{R}_{\infty}\left(\mu_{h_{t}}(z)\right)$ (or with $\beta$ ). Second, we must estimate $N-N_{1}$.

We first consider $N_{1}-\tilde{N}_{1}$. It arises by substituting at least one $\mathscr{R}(\alpha)-\mathscr{R}_{\infty}\left(\mu_{h_{t}}(z)\right)=O\left(k^{-1 / 3}\right)$ for one of the $\mathscr{R}(\alpha)$ 's in $N_{1}$. We apply the Localization Lemma 1.2 to replace $N_{1}$ (and $D$ ) by sums over $\alpha / k, \beta / k \in B\left(\mu_{h_{t}}(z), k^{-1 / 2+\delta}\right)$. We thus need to estimate the following expression, when at least one $\mathscr{R}(\alpha)$ is replaced by $\mathscr{R}(\alpha)-\mathscr{R}_{\infty}\left(\mu_{h_{t}}(z)\right)$ :

$$
\begin{aligned}
& \frac{1}{k} \frac{\sum_{\alpha, \beta} \mathscr{R}_{k}(t, \beta) \mathscr{R}_{k}(t, \alpha)\left(\partial_{t} \log \frac{\mathscr{2}_{h_{t}^{k}}(\beta)}{2_{h_{t}^{k}}(\alpha)}\right)\left(-\partial_{t} \log \mathscr{2}_{h_{t}^{k}}(\alpha)\right) \frac{e^{\langle\alpha, \rho\rangle}}{2_{h_{t}^{k}}(\alpha)} \frac{e^{\langle\beta, \rho\rangle}}{\mathscr{2}_{h_{t}^{k}}(\beta)}}{\left(\sum_{\alpha \in B\left(\mu_{h_{t}}(z), k^{-1 / 2+\delta}\right)} \mathscr{R}_{k}(t, \alpha) \frac{e^{\langle\alpha, \rho\rangle}}{\mathscr{2}_{h_{t}^{k}}(\alpha)}\right)^{2}} \\
& +\frac{1}{k}\left(\frac{\sum_{\alpha, \beta} \mathscr{R}_{k}(t, \beta) \mathscr{R}_{k}(t, \alpha) \partial_{t}^{2} \log \left(\frac{1}{2_{h_{t}^{k}}(\alpha)}\right) \frac{e^{\langle\alpha, \rho\rangle}}{2_{h_{t}^{k}}(\alpha)} \frac{e^{\langle\beta, \rho\rangle}}{2_{h_{t}^{k}}(\beta)}}{\left(\sum_{\alpha \in B\left(\mu_{h_{t}}(z), k^{-1 / 2+\delta}\right)} \mathscr{R}_{k}(t, \alpha) \frac{e^{\langle\alpha, \rho\rangle}}{\left(\mathscr{2}_{h_{t}^{k}}(\alpha)\right)}\right)^{2}}-k \frac{\partial^{2}}{\partial t^{2}} \varphi_{t}\right) .
\end{aligned}
$$

Due to the factor $1 / k$ outside the sum, it suffices to prove that

$$
\left(\left(\frac{\mathscr{T}^{\prime}}{\mathscr{T}}(\alpha)\right)^{\prime}+\frac{1}{2}\left(\frac{\mathscr{T}^{\prime}}{\mathscr{T}}(\alpha)-\frac{\mathscr{T}^{\prime}}{\mathscr{T}}(\beta)\right)^{2}\right)=O\left(k^{1+2 \delta}\right) .
$$


By Proposition 3.1, we have

$$
\frac{\mathscr{T}^{\prime}}{\mathscr{T}}=-\frac{\mathscr{P}^{\prime}}{\mathscr{P}}+k u_{t}^{\prime}\left(\frac{\alpha}{k}\right)
$$

Since $u_{t}=(1-t) u_{0}+t u_{1}$, we have

$$
\frac{\mathscr{T}^{\prime}}{\mathscr{T}}(\alpha)=-\frac{\mathscr{P}^{\prime}}{\mathscr{P}}+k\left(u_{1}-u_{0}\right)\left(\frac{\alpha}{k}\right)=-\frac{\mathscr{P}^{\prime}}{\mathscr{P}}+k\left(f_{1}-f_{0}\right)\left(\frac{\alpha}{k}\right),
$$

where we recall from Section $2 \mathrm{~B}$ that $u_{\varphi}=u_{0}+f_{\varphi}$ with $f_{\varphi}$ smooth up to the boundary of $P$. It follows that

$$
\begin{aligned}
\frac{\mathscr{T}^{\prime}}{\mathscr{T}}(\alpha)-\frac{\mathscr{T}^{\prime}}{\mathscr{T}}(\beta) & =-\frac{\mathscr{P}^{\prime}}{\mathscr{P}}(\alpha)+\frac{\mathscr{P}^{\prime}}{\mathscr{P}}(\beta)+k\left(f_{1}-f_{0}\right)\left(\frac{\alpha}{k}\right)-k\left(f_{1}-f_{0}\right)\left(\frac{\beta}{k}\right), \\
\left(\frac{\mathscr{T}^{\prime}}{\mathscr{T}}(\alpha)\right)^{\prime} & =-\left(\frac{\mathscr{P}^{\prime}}{\mathscr{P}}\right)^{\prime}=O(1),
\end{aligned}
$$

with

$$
k\left(f_{1}-f_{0}\right)\left(\frac{\alpha}{k}\right)-k\left(f_{1}-f_{0}\right)\left(\frac{\beta}{k}\right)=k O\left(\left|\frac{\alpha}{k}-\frac{\beta}{k}\right|\right)=O\left(k^{1 / 2+\delta}\right) .
$$

Further, by Lemma 6.7 (using Proposition 6.6), the factors of

$$
\frac{\left(\frac{\partial}{\partial t}\right) \mathscr{P}_{h_{t}^{k}}(\alpha)}{\mathscr{P}_{h_{t}^{k}}(\alpha)}=\frac{\left(S_{1}(t, \alpha, k)+R_{k}\left(\frac{\alpha}{k}, h\right)\right)}{S_{0}(t, \alpha, k)}=O(1)
$$

and similarly $\left(\mathscr{P}^{\prime} / \mathscr{P}\right)^{\prime}=O(1)$. Since (187) is squared, it has terms as large as $O\left(k^{1+2 \delta}\right)$. Taking into account the overall factor of $\frac{1}{k}$ and the presence of at least one factor of size $k^{-1 / 3}$ coming from the replacement of at least one $\mathscr{R}_{k}(t, \alpha)$ by $\mathscr{R}_{\infty}\left(\mu_{h_{t}}(z)\right)$, we see that $N_{1}-\tilde{N}_{1}$ has order $k^{-(1 / 3)+2 \delta}$ and again this decays for sufficiently small $\delta$.

Now we estimate $N-N_{1}$, which consists of terms with at least one $t$-derivative of $\mathscr{R}$. By Lemma 6.7, the terms with no $t$ derivatives on $\mathscr{T}$ give the terms

$$
\begin{array}{r}
\frac{\sum_{\alpha, \beta} \mathscr{R}_{k}(t, \beta) \mathscr{R}_{k}(t, \alpha)}{}\left(\partial_{t} \log \frac{\mathscr{R}_{k}(t, \alpha)}{\mathscr{R}_{k}(t, \beta)}\right)\left(\partial_{t} \log \mathscr{R}_{k}(t, \alpha)\right) \frac{e^{\langle\alpha, \rho\rangle}}{\mathscr{Q}_{h_{t}^{k}}(\alpha)} \frac{e^{\langle\beta, \rho\rangle}}{\mathscr{2}_{h_{t}^{k}}(\beta)} \\
\left(\sum_{\alpha} \mathscr{R}_{k}(t, \alpha) \frac{e^{\langle\alpha, \rho\rangle}}{\left(\mathscr{Q}_{h_{t}^{k}}(\alpha)\right)}\right)^{2} \\
+\frac{1}{k} \frac{\sum_{\alpha, \beta} \mathscr{R}_{k}(t, \beta) \mathscr{R}_{k}(t, \alpha) \partial_{t}^{2} \log \left(\mathscr{R}_{k}(t, \alpha)\right) \frac{e^{\langle\alpha, \rho\rangle}}{2_{h_{t}^{k}}(\alpha)} \frac{e^{\langle\beta, \rho\rangle}}{\mathscr{2}_{h_{t}^{k}}(\beta)}}{\left(\sum_{\alpha} \mathscr{R}_{k}(t, \alpha) \frac{e^{\langle\alpha, \rho\rangle}}{\left(\mathscr{Q}_{h_{t}^{k}}(\alpha)\right)}\right)^{2}}=O\left(k^{-1}\right),
\end{array}
$$

by Lemma 1.3 .

This leaves us with the terms

$$
\left(\frac{\mathscr{R}^{\prime}}{\mathscr{R}}(\alpha)-\frac{\mathscr{R}^{\prime}}{\mathscr{R}}(\beta)\right)\left(\frac{\mathscr{T}^{\prime}}{\mathscr{T}}(\alpha)-\frac{\mathscr{T}^{\prime}}{\mathscr{T}}(\beta)\right) .
$$


Again by Lemma 6.8, the first term is $O(1)$ while the second factor is (187) and has size $k k^{-1 / 2+\delta}$. Here, we again use Propositions 3.1 and 6.6. Due to the overall factor of $1 / k$ this term has size $k^{-1 / 2+\delta}$.

Therefore, as stated above, up to errors of order $k^{-(1 / 3)+\delta},(184)$ is simplified to $-\partial^{2} \varphi_{t} / \partial t^{2}$ plus

$$
\frac{1}{k}\left(\frac{\sum_{\alpha, \beta} \mathscr{R}_{\infty}\left(\mu_{h_{t}}\left(e^{\rho / 2}\right)\right) \mathscr{R}_{\infty}\left(\mu_{h_{t}}\left(e^{\rho / 2}\right)\right)\left(\partial_{t}^{2} \log \frac{1}{2_{h_{t}^{k}}(\alpha)}+\left(\partial_{t} \log \frac{1}{\mathscr{Q}_{h_{t}^{k}}}\right)\right)\left(\partial_{t} \log \frac{\mathscr{2}_{h_{t}^{k}}(\beta)}{\mathscr{2}_{h_{t}^{k}}(\alpha)}\right) \frac{e^{\langle\alpha, \rho\rangle}}{\mathscr{2}_{h_{t}^{k}}(\alpha)} \frac{e^{\langle\beta, \rho\rangle}}{\mathscr{2}_{h_{t}^{k}}(\beta)}}{\left(\sum_{\alpha \in B\left(\mu_{h_{t}}(z), k^{-1 / 2+\delta}\right)} \mathscr{R}_{\infty}\left(\mu_{h_{t}}\left(e^{\rho / 2}\right)\right) \frac{e^{\langle\alpha, \rho\rangle}}{\left(\mathscr{Q}_{h_{t}^{k}}(\alpha)\right)}\right)^{2}}\right) .
$$

As before, we cancel the factors of $\mathscr{R}_{\infty}\left(\mu_{h_{t}}\left(e^{\rho / 2}\right)\right)$. The resulting difference then cancels to order $k^{-1 / 2+\delta}$ by Proposition 4.5(3).

This completes the proof of the second time derivative estimate, and hence of the main theorem.

\section{Acknowledgments}

The authors thank D. H. Phong and J. Sturm for their support of this project. We also thank them and Y. A. Rubinstein for many detailed corrections. The second author's collaboration with Rubinstein, subsequent to the initial version of this article, has led to a deepened understanding of the global approximation problem, which is reflected in the revised version of the introduction.

\section{References}

[Abreu 2003] M. Abreu, "Kähler geometry of toric manifolds in symplectic coordinates", pp. 1-24 in Symplectic and contact topology: interactions and perspectives (Toronto and Montreal, 2001), edited by Y. Eliashberg et al., Fields Inst. Commun. 35, Amer. Math. Soc., Providence, RI, 2003. MR 2004d:53102

[Arezzo and Tian 2003] C. Arezzo and G. Tian, "Infinite geodesic rays in the space of Kähler potentials", Ann. Sc. Norm. Super. Pisa Cl. Sci. (5) 2:4 (2003), 617-630. MR 2005c:32027

[Berman et al. 2008] R. Berman, B. Berndtsson, and J. Sjöstrand, "A direct approach to Bergman kernel asymptotics for positive line bundles", Ark. Mat. 46:2 (2008), 197-217. MR 2009k:58050 Zbl 1161.32001

[Berndtsson 2009] B. Berndtsson, "Positivity of direct image bundles and convexity on the space of Kähler metrics", J. Differential Geom. 81:3 (2009), 457-482. MR 2010e:32020

[Boutet de Monvel and Sjöstrand 1976] L. Boutet de Monvel and J. Sjöstrand, Sur la singularité des noyaux de Bergman et de Szegó, Astérisque 34-35, Soc. Math. France, Paris, 1976. MR 58 \#28684

[Calabi 1953] E. Calabi, "Isometric imbedding of complex manifolds", Ann. of Math. 58 (1953), 1-23. MR 15,160c Zbl 0051. 13103

[Catlin 1999] D. Catlin, "The Bergman kernel and a theorem of Tian”, pp. 1-23 in Analysis and geometry in several complex variables: Proceedings of the 40th Taniguchi symposium (Katata, 1997), edited by G. Komatsu and M. Kuranishi, Birkhäuser, Boston, 1999. MR 2000e:32001 Zbl 0941.32002

[Chen 2000] X. Chen, “The space of Kähler metrics”, J. Differential Geom. 56:2 (2000), 189-234. MR 2003b:32031

[Chen and Tian 2008] X. X. Chen and G. Tian, "Geometry of Kähler metrics and foliations by holomorphic discs", Publ. Math. Inst. Hautes Études Sci. 107 (2008), 1-107. MR 2009g:32048

[Christ 2003] M. Christ, "Slow off-diagonal decay for Szegö kernels associated to smooth Hermitian line bundles", pp. 77-89 in Harmonic analysis at Mount Holyoke (South Hadley, MA, 2001), edited by W. Beckner et al., Contemp. Math. 320, Amer. Math. Soc., Providence, RI, 2003. MR 2005b:32038 
[Donaldson 1999] S. K. Donaldson, "Symmetric spaces, Kähler geometry and Hamiltonian dynamics”, pp. 13-33 in Northern California Symplectic Geometry Seminar, edited by Y. Eliashberg et al., Amer. Math. Soc. Transl. Ser. 2 196, Amer. Math. Soc., Providence, RI, 1999. MR 2002b:58008

[Donaldson 2001] S. K. Donaldson, "Scalar curvature and projective embeddings, I", J. Differential Geom. 59:3 (2001), 479522. MR 2003j:32030 Zbl 1052.32017

[Donaldson 2002] S. K. Donaldson, "Scalar curvature and stability of toric varieties", J. Differential Geom. 62:2 (2002), 289349. MR 2005c:32028 Zbl 1074.53059

[Donaldson 2005] S. K. Donaldson, "Some numerical results in complex differential geometry", preprint, 2005. arXiv 0512625

[Donaldson 2008] S. K. Donaldson, "Kähler geometry on toric manifolds, and some other manifolds with large symmetry", pp. 29-75 in Handbook of geometric analysis, vol. 1, edited by L. Ji et al., Adv. Lect. Math. (ALM) 7, Int. Press, Somerville, MA, 2008. MR 2483362

[Feng 2009] R. Feng, "Bergman metrics and geodesics in the space of Kähler metrics on principally polarized Abelian varieties”, preprint, 2009. arXiv 0910.2311

[Guan 1999] D. Guan, "On modified Mabuchi functional and Mabuchi moduli space of Kähler metrics on toric bundles”, Math. Res. Lett. 6:5-6 (1999), 547-555. MR 2001b:32042

[Guillemin and Sternberg 1982] V. Guillemin and S. Sternberg, "Convexity properties of the moment mapping", Invent. Math. 67:3 (1982), 491-513. MR 83m:58037 Zbl 0503.58017

[Hörmander 1990] L. Hörmander, The analysis of linear partial differential operators, I: Distribution theory and Fourier analysis, 2nd ed., Grundlehren der Math. Wiss. 256, Springer, Berlin, 1990. MR 91m:35001a Zbl 0712.35001

[Lerman and Tolman 1997] E. Lerman and S. Tolman, "Hamiltonian torus actions on symplectic orbifolds and toric varieties", Trans. Amer. Math. Soc. 349:10 (1997), 4201-4230. MR 98a:57043 Zbl 0897.58016

[Lindholm 2001] N. Lindholm, "Sampling in weighted $L^{p}$ spaces of entire functions in $\mathbb{C}^{n}$ and estimates of the Bergman kernel”, J. Funct. Anal. 182:2 (2001), 390-426. MR 2002g:32007 Zbl 1013.32008

[Mabuchi 1987] T. Mabuchi, "Some symplectic geometry on compact Kähler manifolds. I", Osaka J. Math. 24:2 (1987), 227252. MR $88 \mathrm{~m}: 53126$

[Melin and Sjöstrand 1975] A. Melin and J. Sjöstrand, "Fourier integral operators with complex-valued phase functions", pp. 120-223 in Fourier integral operators and partial differential equations (Colloq. Internat. (Nice, 1974), Lecture Notes in Math. 459, Springer, Berlin, 1975. MR 55 \#4290 Zbl 0306.42007

[Phong and Sturm 2006] D. H. Phong and J. Sturm, "The Monge-Ampère operator and geodesics in the space of Kähler potentials", Invent. Math. 166:1 (2006), 125-149. MR 2007h:32036

[Phong and Sturm 2007] D. H. Phong and J. Sturm, "Test configurations for K-stability and geodesic rays", J. Symplectic Geom. 5:2 (2007), 221-247. MR 2009a:32033 Zbl 05263551

[Phong and Sturm 2009] D. H. Phong and J. Sturm, "Lectures on stability and constant scalar curvature", pp. 101-176 in Current developments in mathematics, 2007, edited by D. Jerison et al., Int. Press, Somerville, MA, 2009. MR 2532997 Zbl 05578296

[Rubinstein 2008] Y. A. Rubinstein, Geometric quantization and dynamical constructions on the space of Kähler metrics, Ph.D. thesis, MIT, 2008, Available at http://dspace.mit.edu/handle/1721.1/44270.

[Rubinstein and Zelditch 2010] Y. A. Rubinstein and S. Zelditch, "Bergman approximations of harmonic maps into the space of Kahler metrics on toric varieties", J. Symplectic Geom. 8:3 (2010), 1-27.

[Rubinstein and Zelditch $\geq 2010$ a] Y. A. Rubinstein and S. Zelditch, in preparation.

[Rubinstein and Zelditch $\geq 2010 \mathrm{~b}$ ] Y. A. Rubinstein and S. Zelditch, "The Cauchy problem for the Monge-Ampère equation, I", in preparation.

[Semmes 1992] S. Semmes, "Complex Monge-Ampère and symplectic manifolds", Amer. J. Math. 114:3 (1992), 495-550. MR 94h:32022 Zbl 0790.32017

[Shiffman and Zelditch 2002] B. Shiffman and S. Zelditch, "Asymptotics of almost holomorphic sections of ample line bundles on symplectic manifolds", J. Reine Angew. Math. 544 (2002), 181-222. MR 2002m:58043 Zbl 1007.53058 
[Shiffman et al. 2003] B. Shiffman, T. Tate, and S. Zelditch, "Harmonic analysis on toric varieties", pp. 267-286 in Explorations in complex and Riemannian geometry, edited by J. Bland et al., Contemp. Math. 332, Amer. Math. Soc., Providence, RI, 2003. MR 2004m:32039 Zbl 1041.32004

[Shiffman et al. 2004] B. Shiffman, T. Tate, and S. Zelditch, "Distribution laws for integrable eigenfunctions", Ann. Inst. Fourier (Grenoble) 54:5 (2004), 1497-1546. MR 2006k:58042 Zbl 1081.35063

[Sjamaar 1995] R. Sjamaar, "Holomorphic slices, symplectic reduction and multiplicities of representations", Ann. of Math. (2) 141:1 (1995), 87-129. MR 96a:58098 Zbl 0827.32030

[Song 2005] J. Song, "The $\alpha$-invariant on certain surfaces with symmetry groups", Trans. Amer. Math. Soc. 357:1 (2005), 45-57. MR 2005g:32031 Zbl 1081.53063

[Song and Zelditch 2007a] J. Song and S. Zelditch, "Convergence of Bergman geodesics on CP1", Ann. Inst. Fourier (Grenoble) 57:7 (2007), 2209-2237. MR 2009k:32025 Zbl 1144.53089

[Song and Zelditch 2007b] J. Song and S. Zelditch, "Test configurations, large deviations and geodesic rays on toric varieties", 2007. arXiv 0712.3599

[Tian 1990] G. Tian, "On a set of polarized Kähler metrics on algebraic manifolds", J. Differential Geom. 32:1 (1990), 99-130. MR 91j:32031

[Yau 1992] S.-T. Yau, "Open problems in geometry", pp. 275-319 in Chern-a great geometer of the twentieth century, edited by S.-T. Yau, Int. Press, Hong Kong, 1992. MR 1201369

[Zelditch 1998] S. Zelditch, "Szegő kernels and a theorem of Tian”, Internat. Math. Res. Notices 1998:6 (1998), 317-331. MR 99g:32055

[Zelditch 2009] S. Zelditch, "Bernstein polynomials, Bergman kernels and toric Kähler varieties", J. Symplectic Geom. 7:2 (2009), 51-76. MR 2496414

Received 2 Mar 2009. Revised 14 Nov 2009. Accepted 14 Dec 2009.

JIAN SONG: jiansong@math.rutgers .edu

Rutgers University, Department of Mathematics, New Brunswick, NJ 08854, United States

STEVE ZeLDITCH: zelditch@math. jhu.edu

Johns Hopkins University, Department of Mathematics, 3400 N. Charles Street, Baltimore, MD 21218, United States

Current address: Northwestern University, Department of Mathematics, 2033 Sheridan Road, Evanston, IL 60208-2370,

United States 


\title{
Analysis \& PDE
}

pjm.math.berkeley.edu/apde

EDITORS

\author{
EDITOR-IN-CHIEF \\ Maciej Zworski \\ University of California \\ Berkeley, USA
}

BOARD OF EDITORS

\begin{tabular}{|c|c|c|c|}
\hline Michael Aizenman & $\begin{array}{l}\text { Princeton University, USA } \\
\text { aizenman@math.princeton.edu }\end{array}$ & Nicolas Burq & $\begin{array}{l}\text { Université Paris-Sud 11, France } \\
\text { nicolas.burq@ math.u-psud.fr }\end{array}$ \\
\hline Luis A. Caffarelli & $\begin{array}{l}\text { University of Texas, USA } \\
\text { caffarel@math.utexas.edu }\end{array}$ & un-Yung Alice Chang & $\begin{array}{l}\text { Princeton University, USA } \\
\text { chang@math.princeton.edu }\end{array}$ \\
\hline Michael Christ & $\begin{array}{l}\text { University of California, Berkeley, USA } \\
\text { mchrist@math.berkeley.edu }\end{array}$ & Charles Fefferman & $\begin{array}{l}\text { Princeton University, USA } \\
\text { cf@math.princeton.edu }\end{array}$ \\
\hline Ursula Hamenstaedt & $\begin{array}{l}\text { Universität Bonn, Germany } \\
\text { ursula@math.uni-bonn.de }\end{array}$ & Nigel Higson & $\begin{array}{l}\text { Pennsylvania State Univesity, USA } \\
\text { higson@ math.psu.edu }\end{array}$ \\
\hline Vaughan Jones & $\begin{array}{l}\text { University of California, Berkeley, USA } \\
\text { vfr@math.berkeley.edu }\end{array}$ & Herbert Koch & $\begin{array}{l}\text { Universität Bonn, Germany } \\
\text { koch@math.uni-bonn.de }\end{array}$ \\
\hline Izabella Laba & $\begin{array}{l}\text { University of British Columbia, Canada } \\
\text { ilaba@math.ubc.ca }\end{array}$ & Gilles Lebeau & $\begin{array}{l}\text { Université de Nice Sophia Antipolis, France } \\
\text { lebeau@unice.fr }\end{array}$ \\
\hline László Lempert & $\begin{array}{l}\text { Purdue University, USA } \\
\text { lempert@math.purdue.edu }\end{array}$ & Richard B. Melrose & $\begin{array}{l}\text { Massachussets Institute of Technology, USA } \\
\text { rbm@math.mit.edu }\end{array}$ \\
\hline Frank Merle & $\begin{array}{l}\text { Université de Cergy-Pontoise, France } \\
\text { Frank.Merle@u-cergy.fr }\end{array}$ & William Minicozzi II & $\begin{array}{l}\text { Johns Hopkins University, USA } \\
\text { minicozz@ math.jhu.edu }\end{array}$ \\
\hline Werner Müller & $\begin{array}{l}\text { Universität Bonn, Germany } \\
\text { mueller@math.uni-bonn.de }\end{array}$ & Yuval Peres & $\begin{array}{l}\text { University of California, Berkeley, USA } \\
\text { peres@stat.berkeley.edu }\end{array}$ \\
\hline Gilles Pisier & $\begin{array}{l}\text { Texas A\&M University, and Paris } 6 \\
\text { pisier@math.tamu.edu }\end{array}$ & Tristan Rivière & $\begin{array}{l}\text { ETH, Switzerland } \\
\text { riviere@math.ethz.ch }\end{array}$ \\
\hline Igor Rodnianski & $\begin{array}{l}\text { Princeton University, USA } \\
\text { irod@math.princeton.edu }\end{array}$ & Wilhelm Schlag & $\begin{array}{l}\text { University of Chicago, USA } \\
\text { schlag@math.uchicago.edu }\end{array}$ \\
\hline Sylvia Serfaty & $\begin{array}{l}\text { New York University, USA } \\
\text { serfaty@ cims.nyu.edu }\end{array}$ & Yum-Tong Siu & $\begin{array}{l}\text { Harvard University, USA } \\
\text { siu@math.harvard.edu }\end{array}$ \\
\hline Terence Tao & $\begin{array}{l}\text { University of California, Los Angeles, USA } \\
\text { tao@math.ucla.edu }\end{array}$ & A Michael E. Taylor & $\begin{array}{l}\text { Univ. of North Carolina, Chapel Hill, USA } \\
\text { met@math.unc.edu }\end{array}$ \\
\hline Gunther Uhlmann & $\begin{array}{l}\text { University of Washington, USA } \\
\text { gunther@math.washington.edu }\end{array}$ & András Vasy & $\begin{array}{l}\text { Stanford University, USA } \\
\text { andras@math.stanford.edu }\end{array}$ \\
\hline Virgil Voiculescu & $\begin{array}{l}\text { University of California, Berkeley, USA } \\
\text { dvv@math.berkeley.edu }\end{array}$ & Steven Zelditch & $\begin{array}{l}\text { Johns Hopkins University, USA } \\
\text { szelditch@math.jhu.edu }\end{array}$ \\
\hline
\end{tabular}

\section{PRODUCTION}

apde@mathscipub.org

Silvio Levy, Scientific Editor

Sheila Newbery, Senior Production Editor

See inside back cover or pjm.math.berkeley.edu/apde for submission instructions.

The subscription price for 2010 is US \$120/year for the electronic version, and \$180/year for print and electronic. Subscriptions, requests for back issues from the last three years and changes of subscribers address should be sent to Mathematical Sciences Publishers, Department of Mathematics, University of California, Berkeley, CA 94720-3840, USA.

Analysis \& PDE, at Mathematical Sciences Publishers, Department of Mathematics, University of California, Berkeley, CA 94720-3840 is published continuously online. Periodical rate postage paid at Berkeley, CA 94704, and additional mailing offices.

APDE peer review and production are managed by EditFLOW ${ }^{\mathrm{TM}}$ from Mathematical Sciences Publishers.

PUBLISHED BY

mathematical sciences publishers

http://www.mathscipub.org

A NON-PROFIT CORPORATION

Typeset in IATE $_{\mathrm{E}} \mathrm{X}$

Copyright $(02010$ by Mathematical Sciences Publishers 


\section{ANALYSIS \& PDE}

\section{Volume $3 \quad$ No. $3 \quad 2010$}

Local WKB construction for Witten Laplacians on manifolds with boundary

Dorian Le PeUtrec

On the Schrödinger equation outside strictly convex obstacles

OANA IVANOVICI

Bergman metrics and geodesics in the space of Kähler metrics on toric varieties

295 JIAN SONG and STEVE ZELDITCH 